\title{
QUANTUM GENERALIZATION OF THE HORN CONJECTURE
}

\author{
PRAKASH BELKALE
}

\section{INTRODUCTION}

Consider the following additive eigenvalue problem for the special unitary group $\mathrm{SU}(n)$ :

- Characterize the possible eigenvalues $(\alpha, \beta, \gamma)$ of traceless Hermitian $n \times n$ matrices $A, B$ and $C$ which satisfy $A+B+C=0$.

(Recall that the Lie algebra of the special unitary group $\mathrm{SU}(n)$ is isomorphic to the real vector space of traceless Hermitian matrices as representations of $\mathrm{SU}(n)$ and hence the terminology "additive eigenvalue problem" for $\mathrm{SU}(n)$.)

In 1962, Horn [17] gave a conjectural solution to a problem equivalent to the above additive eigenvalue problem for $\mathrm{SU}(n)$, by a recursively determined system of inequalities.

In [19], Klyachko gave a solution to the additive eigenvalue problem for $\mathrm{SU}(n)$ in terms of a certain system of inequalities. To write down this system, we need to know which structure constants in the cohomology of $\operatorname{Gr}(r, n)$ (written in the Schubert basis) $0<r<n$ are non-zero, where $\operatorname{Gr}(r, n)$ is the Grassmannian of $r$-dimensional vector subspaces of $\mathbb{C}^{n}$.

By the work of Klyachko and the saturation theorem of Knutson and Tao [20], the problem of determining whether a given structure constant in the cohomology of a Grassmannian $\operatorname{Gr}(r, n)$ (again in the Schubert basis) is non-zero is related to the additive eigenvalue problem for the smaller group $\mathrm{SU}(r)$. Horn's original conjecture followed from these works of Klyachko and of Knutson and Tao. We refer the reader to Fulton's survey article [12] for details.

Our aim in this paper is to formulate and prove an analogue of Horn's conjecture for the following multiplicative eigenvalue problem for the group $\mathrm{SU}(n)$. Let $s \geq 2$ be a positive integer.

- Characterize the possible eigenvalues $\left(\alpha^{1}, \alpha^{2}, \ldots, \alpha^{s}\right)$ of matrices $A^{(1)}, A^{(2)}$, $\ldots, A^{(s)} \in \mathrm{SU}(n)$ which satisfy $A^{(1)} A^{(2)} \cdots A^{(s)}=1$.

1.1. Gromov-Witten numbers and eigenvalue problems. Denote the set of cardinality $r$ subsets $I=\left\{i_{1}<\cdots<i_{r}\right\}$ of $[n]=\{1,2, \ldots, n\}$ by $\left(\begin{array}{c}{[n]} \\ r\end{array}\right)$. To each $I \in\left(\begin{array}{c}{[n]} \\ r\end{array}\right)$ we associate a weakly decreasing sequence of non-negative integers

$$
I \mapsto \lambda(I)=\left(\lambda_{1} \geq \lambda_{2} \geq \cdots \geq \lambda_{r}\right) \in \mathbb{Z}_{\geq 0}^{r},
$$

where $\lambda_{a}=n-r+a-i_{a}$ for $a \in[r]$.

Received by the editors July 22, 2005.

2000 Mathematics Subject Classification. Primary 14N35, 14D20.

The author was partially supported by NSF grant DMS-0300356.

(C) 2007 American Mathematical Society 
Let

$$
E_{\bullet}:\{0\}=E_{0} \subsetneq E_{1} \subsetneq \cdots \subsetneq E_{n}=W
$$

be a complete flag in an $n$-dimensional vector space $W$. For $I \in\left(\begin{array}{c}{[n]} \\ r\end{array}\right)$, the Schubert variety $\Omega_{I}\left(E_{\bullet}\right)$ is defined to be the closed subvariety of the Grassmannian $\operatorname{Gr}(r, W)$ of $r$-dimensional vector subspaces of $W$ given by

$$
\Omega_{I}\left(E_{\bullet}\right)=\left\{V \in \operatorname{Gr}(r, W) \mid \operatorname{dim}\left(V \cap E_{i_{a}}\right) \geq a \text { for } a \in[r]\right\} .
$$

The codimension of $\Omega_{I}\left(E_{\bullet}\right)$ in $\operatorname{Gr}(r, W)$ is $\operatorname{codim}\left(\omega_{I}\right)=\sum_{a=1}^{r}\left(n-r+a-i_{a}\right)$. The cohomology classes $\omega_{I}=\left[\Omega_{I}\left(E_{\bullet}\right)\right]$ form a basis for the integral cohomology $H^{*}(\mathrm{Gr}(r, W), \mathbb{Z})$ of $\operatorname{Gr}(r, W)$.

Fix a set of points $\mathrm{S}=\left\{p_{1}, \ldots, p_{s}\right\}$ on $\mathbb{P}^{1}$. Suppose that we are given $I^{1}, \ldots, I^{s} \in$ $\left(\begin{array}{c}{[n]} \\ r\end{array}\right)$ and a non-negative integer $d$. Let $E_{\bullet}^{1}, \ldots, E_{\bullet}^{s}$ be generic flags on $W$. The Gromov-Witten number $\left\langle\omega_{I^{1}}, \ldots, \omega_{I^{s}}\right\rangle_{d}$ is defined to be the number of maps $f$ : $\mathbb{P}^{1} \rightarrow G r(r, W)$ of degree $d$ such that $f\left(p_{j}\right) \in \Omega_{I^{j}}\left(E_{\bullet}^{j}\right)$ for $j=1, \ldots, s$. If there is an infinite number of such maps, $\left\langle\omega_{I^{1}}, \ldots, \omega_{I^{s}}\right\rangle_{d}$ is defined to be zero.

Conjugacy classes in the special unitary group $\mathrm{SU}(n)$ are in one to one correspondence with points in the $(n-1)$-simplex

$$
\Delta(n)=\left\{\alpha=\left(\alpha_{1}, \ldots, \alpha_{n}\right) \mid \alpha_{1} \geq \cdots \geq \alpha_{n} \geq \alpha_{1}-1, \sum_{t=1}^{n} \alpha_{t}=0\right\} \subseteq \mathbb{R}^{n}
$$

where, to $\left(\alpha_{1}, \ldots, \alpha_{n}\right)$, we associate the conjugacy class of the diagonal matrix with entries $\exp \left(2 \pi \sqrt{-1} \alpha_{t}\right)$ for $t=1, \ldots, n$.

The following generalization of Klyachko's solution of the additive eigenvalue problem [19] was proven independently by Agnihotri and Woodward [2] and the author [4] (also see [8]). It says that the multiplicative eigenvalue problem for $\mathrm{SU}(n)$ is controlled by the quantum Schubert calculus of the Grassmannians $\operatorname{Gr}(r, n)$.

Theorem 1.1. Let $\alpha^{1}, \ldots, \alpha^{s}$ be conjugacy classes in $\mathrm{SU}(n)$ with $\alpha^{j}=\left(\alpha_{1}^{j}, \ldots, \alpha_{n}^{j}\right)$ for $j=1, \ldots, s$. The following are equivalent:

(1) There exist $A^{(1)}, \ldots, A^{(s)} \in \mathrm{SU}(n)$ with $A^{(j)}$ in the conjugacy class $\alpha^{j}$ such that $A^{(1)} A^{(2)} \cdots A^{(s)}=1$.

(2) For any integers $r, d$ with $0<r<n, d \geq 0$, and $I^{1}, \ldots, I^{s} \in\left(\begin{array}{c}{[n]} \\ r\end{array}\right)$, such that $\left\langle\omega_{I^{1}}, \ldots, \omega_{I^{s}}\right\rangle_{d} \neq 0$, the following inequality holds:

$$
\sum_{j=1}^{s} \sum_{t \in I^{j}} \alpha_{t}^{j} \leq d
$$

\subsection{The multiplicative Horn problem.}

Definition 1.2. For $I \in\left(\begin{array}{c}{[n]} \\ r\end{array}\right)$ where $0<r<n$, with associated sequence $\lambda(I)=$ $\left(\lambda_{1} \geq \cdots \geq \lambda_{r}\right)$, define a conjugacy class $\beta(I)=\left(\beta_{1}, \ldots, \beta_{r}\right)$ for $\mathrm{SU}(r)$ as follows:

$$
\beta(I)=\frac{1}{n-r}\left(\lambda_{1}, \ldots, \lambda_{r}\right)-\frac{|\lambda(I)|}{r(n-r)}(1, \ldots, 1)
$$

where $|\lambda(I)|=\sum_{a=1}^{r} \lambda_{a}$. More explicitly, for $a \in[r]$,

$$
\beta_{a}=\frac{1}{n-r}\left(\lambda_{a}-\frac{1}{r}|\lambda(I)|\right) .
$$


The center of $\mathrm{SU}(r)$ acts on the conjugacy classes of elements in $\mathrm{SU}(r)$. Set $\zeta_{r}=\exp \left(\frac{2 \pi \sqrt{-1}}{r}\right) \in \mathbb{C}$. Given a conjugacy class $\alpha$ for $\mathrm{SU}(r)$, we can multiply $\alpha$ by $\zeta_{r}$ and obtain a new conjugacy class $\zeta_{r} \alpha$. Explicitly, if $\alpha=\left(\alpha_{1}, \ldots, \alpha_{r}\right)$, then $\zeta_{r} \alpha=\left(\alpha_{2}+\frac{1}{r}, \ldots, \alpha_{r}+\frac{1}{r}, \alpha_{1}+\frac{1}{r}-1\right)$. The main theorem of this paper is the following:

Theorem 1.3. Let $I^{1}, \ldots, I^{s} \in\left(\begin{array}{c}{[n]} \\ r\end{array}\right)$, where $0<r<n$, and let $d$ be a non-negative integer. Assume that

$$
\sum_{j=1}^{s} \operatorname{codim}\left(\omega_{I^{j}}\right)=r(n-r)+d n .
$$

The following are equivalent:

(1) $\left\langle\omega_{I^{1}}, \ldots, \omega_{I^{s}}\right\rangle_{d} \neq 0$.

(2) There exist $A^{(1)}, \ldots, A^{(s)} \in \mathrm{SU}(r)$ such that

- $A^{(1)} A^{(2)} \cdots A^{(s)}=1$.

- $A^{(1)}$ is in the conjugacy class $\zeta_{r}^{d} \beta\left(I^{1}\right)$ and for $j \geq 2, A^{(j)}$ is in the conjugacy class $\beta\left(I^{j}\right)$.

(3) There exists an $\mathrm{SU}(n)$-local system $\mathcal{L}$ on $\mathbb{P}^{1}-\mathrm{S}$ such that the local monodromy of $\mathcal{L}$ at $p_{1}$ is $\zeta_{r}^{d} \beta\left(I^{1}\right)$ and the monodromy at $p_{j}$ for $j \geq 2$ is $\beta\left(I^{j}\right)$.

The equivalence of (2) and (3) follows immediately from the description of the fundamental group of $\mathbb{P}^{1}-\mathrm{S}$ as a free group on generators $\gamma_{1}, \gamma_{2}, \ldots, \gamma_{s}$ modulo the relation $\gamma_{1} \gamma_{2} \cdots \gamma_{s}=1$.

The implication $(1) \Rightarrow(3)$ is a remarkable way of producing unitary local systems on $\mathbb{P}^{1}-\mathrm{S}$ from non-vanishing Gromov-Witten numbers. This implication can also be obtained from the work of Witten [33] and Agnihotri [1] as was pointed out to us by Woodward.

In Theorem 1.5, we will give an explicit system of inequalities which is equivalent to the criteria in Theorem 1.3.

1.2.1. Consequences of Theorem 1.3 for eigenvalue problems. Define subsets $\Gamma(n, s)$ $\subseteq \Delta(n)^{s}$ for positive integers $n$ (and the fixed positive integer $s$ ), recursively, as follows:

(1) $\Gamma(1, s)=\Delta(1)^{s}$.

(2) Let $n$ be a positive integer. Assume $\Gamma(r, s)$ has been defined whenever $0<r<n$. Define $\Gamma(n, s)$ as follows: $\left(\alpha^{1}, \ldots, \alpha^{s}\right) \in \Gamma(n, s)$ where $\alpha^{j}=$ $\left(\alpha_{1}^{j}, \ldots, \alpha_{n}^{j}\right)$ if and only if for any integers $r$ and $d$ with $0<r<n, d \geq 0$, and $I^{1}, \ldots, I^{s} \in\left(\begin{array}{c}{[n]} \\ r\end{array}\right)$, such that

- $\sum_{j=1}^{s} \sum_{a=1}^{r}\left(n-r+a-i_{a}^{j}\right)=r(n-r)+d n$,

- $\left(\zeta_{r}^{d} \beta\left(I^{1}\right), \beta\left(I^{2}\right), \ldots, \beta\left(I^{s}\right)\right) \in \Gamma(r, s)$,

the following inequality holds:

$$
\sum_{j=1}^{s} \sum_{t \in I^{j}} \alpha_{t}^{j} \leq d .
$$

The following corollary is immediate from Theorem 1.3 and Theorem 1.1 and is the multiplicative ("quantum") generalization of Horn's conjecture:

Corollary 1.4. Let $\alpha^{1}, \ldots, \alpha^{s} \in \Delta(n)$ be conjugacy classes in $\mathrm{SU}(n)$. Then there exist $A^{(1)}, \ldots, A^{(s)} \in \mathrm{SU}(n)$ with $A^{(j)}$ in the conjugacy class $\alpha^{j}$ and $A^{(1)} A^{(2)} \cdots A^{(s)}$ $=1$ if and only if $\left(\alpha^{1}, \ldots, \alpha^{s}\right) \in \Gamma(n, s)$. 
1.3. Quantum product of Schubert classes. In this section we will state results on the small quantum cohomology of the Grassmannian $\operatorname{Gr}(r, n)$, where $0<r<n$. Recall that the small quantum cohomology of $\operatorname{Gr}(r, n)$, denoted by $\mathrm{QH}^{*}(\operatorname{Gr}(r, n))$, is an associative ring whose underlying abelian group is $H^{*}(\operatorname{Gr}(r, n), \mathbb{Z}) \otimes \mathbb{Z}[q]$ and the $\mathbb{Z}[q]$-linear product structure is given by

$$
\omega_{I} \star \omega_{J}=\sum_{d} \sum_{K}\left\langle\omega_{I}, \omega_{J}, \omega_{K}\right\rangle_{d} q^{d} \omega_{K^{\prime}}
$$

where the first sum is over all non-negative integers $d$ and the second sum is over all $K \in\left(\begin{array}{c}{[n]} \\ r\end{array}\right)\left(\omega_{K^{\prime}}\right.$ is the dual of $\omega_{K}$; see Section 2.1).

The classes $q^{d} \omega_{I}$ form an additive basis for the quantum cohomology ring $\mathrm{QH}^{*}(\operatorname{Gr}(r, n))$ where $d$ varies over all non-negative integers and $I$ varies over all elements of $\left(\begin{array}{c}{[n]} \\ r\end{array}\right)$. Given any element $\tau \in \mathrm{QH}^{*}(\operatorname{Gr}(r, n))$, we say that $q^{d}$ appears in $\tau$ if the coefficient of $q^{d} \omega_{I}$ in $\tau$ is non-zero for some $I$.

There is a natural grading on quantum cohomology: The degree of $q$ is $n$ and non-zero elements in $H^{2 k}(\operatorname{Gr}(r, n))$ are homogeneous of degree $k$. We refer the reader to [22], [10] for the algebro-geometric foundations of quantum cohomology.

Let $I^{1}, \ldots, I^{s} \in\left(\begin{array}{c}{[n]} \\ r\end{array}\right)$, and let $d$ be a non-negative integer. We will give a criterion to determine if $q^{c}$ appears in $\omega_{I^{1}} \star \cdots \star \omega_{I^{s}}$ for some $c \leq d$. The case of two factors for an arbitrary $G / P$ was considered by Fulton and Woodward in [11]. Recall that (by degree considerations), if $\sum_{j=1}^{s} \operatorname{codim}\left(\omega_{I^{j}}\right)>d n+r(n-r)$ and $c \leq d$, then $q^{c}$ does not appear in $\omega_{I^{1}} \star \cdots \star \omega_{I^{s}}$.

Theorem 1.5. Let $I^{1}, \ldots, I^{s} \in\left(\begin{array}{c}{[n]} \\ r\end{array}\right)$, and let d be a non-negative integer such that

$$
\sum_{j=1}^{s} \operatorname{codim}\left(\omega_{I^{j}}\right) \leq d n+r(n-r) .
$$

Write $d=q r+b$ with $0 \leq b<r$ and $(q, b) \in \mathbb{Z}^{2}$. Let

$$
L=\left\{x \in[n] \mid \exists y \in I^{1}, x \equiv y-i_{b}^{1} \quad(\bmod n)\right\}
$$

(where $i_{b}^{1}=0$ if $\left.b=0\right)$. Let $\left(\tilde{\lambda}_{1}, \ldots, \tilde{\lambda}_{r}\right)=\lambda(L)$ and $\left(\lambda_{1}^{j}, \ldots, \lambda_{r}^{j}\right)=\lambda\left(I^{j}\right)$ for $j=2, \ldots, s$. The following are equivalent:

(a) For some $c \leq d$, $q^{c}$ appears in $\omega_{I^{1}} \star \cdots \star \omega_{I^{s}}$.

(b) For any integers $d^{\prime}$ and $r^{\prime}$ with $0<r^{\prime}<r, d^{\prime} \geq 0$, and $K^{1}, \ldots, K^{s} \in\left(\begin{array}{c}{[r]} \\ r^{\prime}\end{array}\right)$ such that $\left\langle\omega_{K^{1}}, \ldots, \omega_{K^{s}}\right\rangle_{d^{\prime}}=1$, the following inequality holds:

$$
\sum_{a \in K^{1}} \tilde{\lambda}_{a}+\sum_{j=2}^{s} \sum_{a \in K^{j}} \lambda_{a}^{j} \leq d^{\prime}(n-r)+r^{\prime}\left(q n+i_{b}^{1}\right)+r^{\prime}(n-r) .
$$

If equality holds in inequality (1.3), then $(a)$ and $(b)$ are equivalent to

(c) $\left\langle\omega_{I^{1}}, \ldots, \omega_{I^{s}}\right\rangle_{d} \neq 0$.

Theorem 1.5 gives some information on the smallest power of $q$ that appears in a quantum product $\omega_{I^{1}} \star \cdots \star \omega_{I^{s}}$. We have not been able to turn this into a combinatorial characterization of the smallest power, as Fulton and Woodward did ([11], Proposition 9.1(2)) in the case $s=2$. It should be noted that Theorem 1.3 gives no information on the other powers of $q$ that appear in the same product. In the case $s=2$, Yong [34] has found an upper bound for the highest power of $q$ appearing in $\omega_{I} \star \omega_{J}$, and Postnikov [28] has found exactly which $c$ have $q^{c}$ appear in $\omega_{I} \star \omega_{J}$. Analogous results for $s>2$ are not known. 
1.4. Transversality. Fix an algebraically closed field $\kappa$ of arbitrary characteristic. For integers $r$ and $n$ with $0<r<n$, let $\operatorname{Gr}(r, n)$ be the Grassmannian of $r$ dimensional subspaces of $\kappa^{n}$ and let $\mathcal{M}_{r, n}^{d}$ be the space of maps $\mathbb{P}^{1} \rightarrow \operatorname{Gr}(r, n)$ of degree $d \geq 0$ over $\kappa$. It is known that $\mathcal{M}_{r, n}^{d}$ is a smooth quasi-projective variety of dimension $r(n-r)+d n$ (see e.g. [10]). Let $\mathrm{S}=\left\{p_{1}, \ldots, p_{s}\right\} \subset \mathbb{P}^{1}(\kappa)$. There is a natural morphism

$$
\pi: \mathcal{M}_{r, n}^{d} \rightarrow \prod_{j=1}^{s} \operatorname{Gr}(r, n), \pi(f)=\left(f\left(p_{1}\right), \ldots, f\left(p_{s}\right)\right) .
$$

Let $I^{1}, \ldots, I^{s} \in\left(\begin{array}{c}{[n]} \\ r\end{array}\right)$ and assume that

$$
\sum_{j=1}^{s} \operatorname{codim}\left(\omega_{I^{j}}\right)=r(n-r)+d n .
$$

Theorem 1.6. For generic flags $E_{\bullet}^{j}$ on $\kappa^{n}$, the scheme $\pi^{-1}\left(\prod_{j=1}^{s} \Omega_{I^{j}}\left(E_{\bullet}^{j}\right)\right)$ is a finite reduced scheme (possibly empty).

If $\kappa$ is a field of characteristic 0, then the theorem follows from Kleiman's transversality theorem [18]. If $\kappa$ is of positive characteristic, then Kleiman's results give only the finiteness of $\pi^{-1}\left(\prod_{j=1}^{s} \Omega_{I^{j}}\left(E_{\bullet}^{j}\right)\right)$.

The case where are all $I^{j}$ are the same and correspond to divisors (that is, $\left.I^{j}=\{n-r, n-r+2, n-r+3, \ldots, n\}\right)$ is due to Sottile [31]. The classical part (that is, $d=0$ ) of Theorem 1.6 was proven independently by the author [6] and Vakil [32]. The proof of Theorem 1.6 is extracted from the geometric ideas underlying the proof of Theorem 1.3 following a suggestion of Sottile.

The interesting problem of finding the maximum possible number of real or $p$ adic solutions to a "quantum Schubert enumerative problem" remains open (but see [30], [32]).

1.5. Saturation for fusion structure coefficients. In [6], the author gave a geometric proof of Horn's conjecture and using the classical relation between the cohomology of the Grassmannians $\operatorname{Gr}(r, n)$ and the invariant theory of $\mathrm{SU}(r)$ (or equivalently $\mathrm{SL}(r)$ ) gave a new proof of the saturation theorem of Knutson and Tao [20].

The fusion ring, also called the Verlinde algebra in the physics literature, of $\mathrm{SU}(r)$ (at a certain level) is the quantum analogue of the representation ring of $\mathrm{SU}(r)$, and the (small) quantum cohomology of $\operatorname{Gr}(r, n)$ is of course the quantum analogue of the usual cohomology of $\operatorname{Gr}(r, n)$. A theorem of Witten [33] relates the (small) quantum cohomology of Grassmannians to the fusion rings of unitary groups. So it is natural to expect that a quantum saturation theorem would follow from our analogue of Horn's conjecture. Before stating our theorem, we recall some definitions related to the fusion ring of $\mathrm{SU}(r)$ (see e.g. [3] for the basic theory).

Irreducible polynomial representations of the unitary group $\mathrm{U}(r)$ are parameterized by weakly decreasing sequences of non-negative integers $\lambda=\left(\lambda_{1} \geq \lambda_{2} \geq\right.$ $\left.\cdots \geq \lambda_{r}\right)$. These restrict to irreducible representations $\bar{\lambda}$ of $\mathrm{SU}(r)$. Sequences $\lambda$ and $\mu$ restrict to give the same irreducible representation of $\mathrm{SU}(r)$ if and only if the difference $\lambda_{a}-\mu_{a}=c$ for some constant $c$ and all $a \in[r]$. The congruence class $|\bar{\mu}|=\sum_{a=1}^{r} \mu_{a}(\bmod r) \in \mathbb{Z} / r \mathbb{Z}$ is therefore well defined. 
Let $k$ be a positive integer. Following the notation in [3], consider the set $P_{k}$ of irreducible representations $\bar{\mu}$ of $\mathrm{SU}(r)$ such that $\mu_{1}-\mu_{r} \leq k$. Given $\bar{\mu}^{1}, \ldots, \bar{\mu}^{s} \in P_{k}$, let $N_{0}^{(k)}\left(\bar{\mu}^{1}, \ldots, \bar{\mu}^{s}\right)$ denote the dimension of the corresponding vector space of conformal blocks for genus 0 at level $k$. This number is also the dimension of the space of sections of a certain line bundle on a moduli stack of parabolic bundles on $\mathbb{P}^{1}[27]$.

Remark 1.7. If $k \geq \sum_{j=1}^{s}\left(\mu_{1}^{j}-\mu_{r}^{j}\right)$, then $N_{0}^{(k)}\left(\bar{\mu}^{1}, \ldots, \bar{\mu}^{s}\right)$ coincides with the dimension of the space of $\mathrm{SU}(r)$ invariants in the tensor product of the representations $\bar{\mu}^{1}, \ldots, \bar{\mu}^{s}$.

The fusion ring of $\mathrm{SU}(r)$ at level $k$, denoted by $R(\mathrm{SU}(r))_{k}$, is an associative ring whose underlying abelian group is freely generated by representations $\bar{\nu} \in P_{k}$, and the product is given by

$$
\bar{\mu}^{1} \cdot \bar{\mu}^{2} \cdots \bar{\mu}^{s}=\sum_{\bar{\nu}} N_{0}^{(k)}\left(\bar{\mu}^{1}, \ldots, \bar{\mu}^{s}, \bar{\nu}\right) \bar{\nu}^{*}
$$

where $\bar{\nu}$ varies over all elements of $P_{k}$ and $\bar{\nu}^{*}$ is the dual of $\bar{\nu}$.

Let $\bar{\mu}^{1}, \ldots, \bar{\mu}^{s} \in P_{k}$ be such that $\sum_{j=1}^{s}\left|\bar{\mu}^{j}\right|=0 \in \mathbb{Z} / r \mathbb{Z}$, and let $M$ be a nonnegative integer. Then

Theorem 1.8. $N_{0}^{(k)}\left(\bar{\mu}^{1}, \ldots, \bar{\mu}^{s}\right) \neq 0$ if and only if $N_{0}^{(M k)}\left(M \bar{\mu}^{1}, \ldots, M \bar{\mu}^{s}\right) \neq 0$.

(Note that $R(\mathrm{SU}(r))_{k}$ can be realized as a quotient of the representation ring of $\mathrm{SU}(r)$ and hence if $\sum_{j=1}^{s}\left|\bar{\mu}^{j}\right| \neq 0 \in \mathbb{Z} / r \mathbb{Z}$, then $N_{0}^{(k)}\left(\bar{\mu}^{1}, \ldots, \bar{\mu}^{s}\right)=0$.)

Theorem 1.8 is a generalization of the Knutson-Tao saturation theorem: Given irreducible representations $\bar{\mu}^{1}, \ldots, \bar{\mu}^{s}$ of $\mathrm{SU}(r)$, such that $\sum_{j=1}^{s}\left|\bar{\mu}^{j}\right|=0 \in \mathbb{Z} / r \mathbb{Z}$, fix an integer $k$ such that $k \geq \sum_{j=1}^{s}\left(\mu_{1}^{j}-\mu_{r}^{j}\right)$. Clearly, $\bar{\mu}^{1}, \ldots, \bar{\mu}^{s} \in P_{k}$. By Remark 1.7, $N_{0}^{(k)}\left(\bar{\mu}^{1}, \ldots, \bar{\mu}^{s}\right)\left(\right.$ resp. $\left.N_{0}^{(M k)}\left(M \bar{\mu}^{1}, \ldots, M \bar{\mu}^{s}\right)\right)$ coincides with the dimension of the space of $\mathrm{SU}(r)$ invariants in the tensor product of the representations $\bar{\mu}^{1}, \ldots, \bar{\mu}^{s}$ (resp. $\left.M \bar{\mu}^{1}, \ldots, M \bar{\mu}^{s}\right)$. Hence, we recover the saturation theorem, which asserts that one of these spaces of invariants is non-zero if and only if the other is non-zero.

1.6. An overview of the methods. It is standard that we can view $\left\langle\omega_{I^{1}}, \ldots, \omega_{I^{s}}\right\rangle_{d}$ from Section 1.1 also as the number of subbundles of degree $-d$ and rank $r$ (if finite and zero otherwise) $\mathcal{V}$ of the trivial vector bundle $\mathcal{W}=\mathcal{O}_{\mathbb{P}^{1}}^{n}$ on $\mathbb{P}^{1}$ of rank $n$, such that $\mathcal{V}_{p_{j}} \in \Omega_{I^{j}}\left(E_{\bullet}^{j}\right)$ for $j=1, \ldots, s$. To get an inductive grip on this situation, we would like to replace $\mathcal{W}$ by $\mathcal{V}$. But $\mathcal{V}$ may not be trivial as a vector bundle. However, it can be shown that $\mathcal{V}$ is "evenly split" (see Section 2.3 and Lemma 4.5). This motivates us to carry out a generalization of Gromov-Witten numbers (Section 2.4).

With this inductive framework in place, the strategy for the proofs is very similar to those in [6] (where many of the arguments in this paper appear in a simpler situation): The tangent space technique is modified to apply in a more general situation. Standard properties of quot schemes are used to do the tangent space calculations. Some general position techniques from [6] are also used.

There are some additional difficulties in the quantum situation arising from the nature of morphisms between vector bundles on $\mathbb{P}^{1}$ (e.g. the image of a morphism of vector bundles may not be a subbundle). We use techniques inspired by the theory of parabolic bundles to overcome these difficulties. 
1.7. Conventions. All schemes in this paper are assumed to be of finite type over an algebraically closed base field $\kappa$ of arbitrary characteristic.

(1) Fix a finite collection of points $\mathrm{S}=\left\{p_{1}, \ldots, p_{s}\right\}$ on $\mathbb{P}^{1}$.

(2) The (small) category of sets will be denoted by (Sets).

(3) A morphism $\mathcal{V} \rightarrow \mathcal{W}$ of locally free sheaves on a scheme $X$ is said to have rank $r$ if the cokernel is a locally free sheaf of $\operatorname{rank} \operatorname{dim}(\mathcal{W})-r$.

(4) For a weakly decreasing sequence of non-negative integers $\lambda=\left(\lambda_{1} \geq \lambda_{2} \geq\right.$ $\left.\cdots \geq \lambda_{r}\right)$, let $|\lambda|=\sum_{a=1}^{r} \lambda_{a}$.

\section{Formulation of the main Result}

2.1. Schubert cells. Let

$$
E_{\bullet}:\{0\}=E_{0} \subsetneq E_{1} \subsetneq \cdots \subsetneq E_{n}=W
$$

be a complete flag in an $n$-dimensional vector space $W$. For $I \in\left(\begin{array}{c}{[n]} \\ r\end{array}\right)$, define the Schubert cell $\Omega_{I}^{o}\left(E_{\bullet}\right) \subseteq \operatorname{Gr}(r, W)$ by

$$
\Omega_{I}^{o}\left(E_{\bullet}\right)=\left\{V \in \operatorname{Gr}(r, W) \mid \operatorname{dim}\left(V \cap E_{u}\right)=a \text { for } i_{a} \leq u<i_{a+1}, a=0, \ldots, r\right\}
$$

where $i_{0}$ is defined to be 0 and $i_{r+1}=n$. It is easy to see that $\Omega_{I}\left(E_{\bullet}\right)$ (defined in the introduction) is the closure of $\Omega_{I}^{o}\left(E_{\bullet}\right)$.

The dual of the cohomology class $\omega_{I}$ of $\Omega_{I}\left(E_{\bullet}\right)$ under the intersection pairing is $\omega_{I^{\prime}}$ where $I^{\prime}=\{i \in[n] \mid n+1-i \in I\}$. This means that if $\operatorname{codim}\left(\omega_{I}\right)+\operatorname{codim}\left(\omega_{J}\right)=$ $r(n-r)$, the intersection number $\omega_{I} \cdot \omega_{J} \in H^{2 r(n-r)}(\operatorname{Gr}(r, W), \mathbb{Z})=\mathbb{Z}$ is 1 if $J=I^{\prime}$ and 0 otherwise.

2.2. Complete flags. For a vector bundle $\mathcal{W}$ on $\mathbb{P}^{1}$, define

$$
\mathrm{Fl}_{\mathrm{S}}(\mathcal{W})=\prod_{p \in \mathrm{S}} \mathrm{Fl}\left(\mathcal{W}_{p}\right)
$$

If $\mathcal{E} \in \mathrm{Fl}_{\mathrm{S}}(\mathcal{W})$, we will assume that it is written in the form $\mathcal{E}=\left(E_{\bullet}^{p} \mid p \in \mathrm{S}\right)$. More generally if $T$ is a scheme and $\mathcal{W}$ is a vector bundle on $\mathbb{P}^{1} \times T$, let $\mathrm{Fl}_{\mathrm{S}}(\mathcal{W})$ be the scheme over $T$, consisting of pairs $(t, \mathcal{E})$ where $t \in T$ and $\mathcal{E} \in \mathrm{Fl}_{\mathrm{S}}\left(\mathcal{W}_{t}\right)$.

For a vector bundle $\mathcal{W}$ on $\mathbb{P}^{1}$, a subbundle $\mathcal{V} \subseteq \mathcal{W}$ and $\mathcal{E}=\left(E_{\bullet}^{p} \mid p \in \mathrm{S}\right) \in$ $\mathrm{Fl}_{\mathrm{S}}(\mathcal{W})$ we have associated induced complete flags on the fibres at $p \in \mathrm{S}$ of $\mathcal{V}$ and $\mathcal{Q}=\mathcal{W} / \mathcal{V}$. We denote these by $\mathcal{E}(\mathcal{V})=\left(E_{\bullet}^{p}(\mathcal{V}) \mid p \in \mathrm{S}\right) \in \mathrm{Fl}_{\mathrm{S}}(\mathcal{V})$ and $\mathcal{E}(\mathcal{Q})=\left(E_{\bullet}^{p}(\mathcal{Q}) \mid p \in \mathrm{S}\right) \in \mathrm{Fl}_{\mathrm{S}}(\mathcal{Q})$

2.3. Evenly split vector bundles on $\mathbb{P}^{1}$. A vector bundle $\mathcal{W}$ on $\mathbb{P}^{1}$ is said to be evenly split if $\mathcal{W}=\bigoplus_{i=1}^{n} \mathcal{O}_{\mathbb{P}^{1}}\left(a_{i}\right)$ with $\left|a_{i}-a_{j}\right| \leq 1$ for $0<i<j \leq n$.

Let $D$ and $n$ be integers with $n>0$. It is easy to show that up to isomorphism, there is a unique evenly split vector bundle of degree $-D$ and rank $n$ on $\mathbb{P}^{1}$. We denote this vector bundle by $\mathcal{Z}_{D, n}$.

Let $\mathcal{W}$ be a vector bundle on $\mathbb{P}^{1}$. Let $d$ and $r$ be integers with $r \geq 0$ (and $d$ possibly negative). Define $\operatorname{Gr}(d, r, \mathcal{W})$ to be the moduli space of subbundles of $\mathcal{W}$ of degree $-d$ and rank $r$. This is an open subset of the quot scheme of quotients of $\mathcal{W}$ of degree $d-D$ and rank $n-r$.

Definition 2.1. For non-negative integers $r, m$ and for $b, d \in \mathbb{Z}$ let

$$
\chi(d, r, b, m)=\chi\left(\mathbb{P}^{1}, \mathcal{H o m}\left(\mathcal{Z}_{d, r}, \mathcal{Z}_{b, m}\right)\right)=r m+d m-b r .
$$

Proposition 2.2. (1) $\operatorname{Gr}\left(d, r, \mathcal{Z}_{D, n}\right)$ is smooth and connected (possibly empty) of dimension $\chi(d, r, D-d, n-r)$. 
(2) The subset of $\operatorname{Gr}\left(d, r, \mathcal{Z}_{D, n}\right)$ consisting of evenly split subbundles $\mathcal{V} \subseteq \mathcal{Z}_{D, n}$ such that $\mathcal{Z}_{D, n} / \mathcal{V}$ is also evenly split is open and dense in $\operatorname{Gr}\left(d, r, \mathcal{Z}_{D, n}\right)$.

The proof of Proposition 2.2 (which is well known) will be given in Section 12.1.

\subsection{Schubert states and generalized Gromov-Witten numbers.}

Definition 2.3. A Schubert state is a 5 -tuple $\mathcal{I}=(d, r, D, n, I)$ where $d, D, r$ and $n$ are integers, $0 \leq r \leq n$, and $I$ assigns to each $p \in \mathrm{S}$ an element $I^{p} \in\left(\begin{array}{c}{[n]} \\ r\end{array}\right)$ (we allow the possibilities $r=0$ and $r=n)$. We will use the notation $I^{p}=\left\{i_{1}^{p}<\cdots<i_{r}^{p}\right\}$ for $p \in \mathrm{S}$.

Let $\mathcal{W}=\mathcal{Z}_{D, n}$ and let $\mathcal{I}=(d, r, D, n, I)$ be a Schubert state. Fix a generic point $\mathcal{E} \in \mathrm{Fl}_{\mathrm{S}}(\mathcal{W})$. For $p \in \mathrm{S}$, let $\pi_{p}: \operatorname{Gr}(d, r, \mathcal{W}) \rightarrow \operatorname{Gr}\left(r, \mathcal{W}_{p}\right)$ be the natural morphism (the fibre of the subbundle at $p$ ). For $p \in \mathrm{S}$, the group $\mathrm{GL}\left(\mathcal{W}_{p}\right)$ acts transitively on $\operatorname{Gr}\left(r, \mathcal{W}_{p}\right)$. Hence by Kleiman's transversality theorem [18], the dimension of each irreducible component of the intersection

$$
\bigcap_{p \in \mathrm{S}} \pi_{p}^{-1}\left(\Omega_{I^{p}}^{o}\left(E_{\bullet}^{p}\right)\right) \subseteq \operatorname{Gr}(d, r, \mathcal{W})
$$

equals

$$
\operatorname{dim} \mathcal{I}=\operatorname{dim} \operatorname{Gr}\left(d, r, \mathcal{Z}_{D, n}\right)-\sum_{p \in \mathrm{S}} \operatorname{codim}\left(\omega_{I^{p}}\right) .
$$

Definition 2.4. (1) Define $\langle\mathcal{I}\rangle$ to be the number of points in the intersection (2.1) (if finite and 0 otherwise).

(2) We will say that $\mathcal{I}$ is non-null (resp. null) if (2.1) is a possibly infinite non-empty intersection (resp. empty).

Remark 2.5. If $D=0$, then $\langle\mathcal{I}\rangle=\left\langle\omega_{I^{p_{1}}}, \ldots, \omega_{I^{p_{s}}}\right\rangle_{d}$.

If the base field is of characteristic 0 and $\operatorname{dim} \mathcal{I}=0$, it follows from Kleiman's transversality theorem that the intersection (2.1) is a reduced zero-dimensional scheme. In Proposition 10.1, we will show that this property holds for any algebraically closed field.

2.5. Shift operations. The following two results on shift operations are proved in Section 11.

Lemma 2.6. Consider a Schubert state $\mathcal{I}=(d, r, D, n, I)$. Set $\mathcal{J}=(d+r, r, D+$ $n, n, I)$. Then

(1) $\operatorname{dim} \mathcal{I}=\operatorname{dim} \mathcal{J}$,

(2) $\mathcal{I}$ is non-null $\Leftrightarrow \mathcal{J}$ is non-null,

(3) $\langle\mathcal{I}\rangle=\langle\mathcal{J}\rangle$.

Consider the operator Sh acting on $\left(\begin{array}{c}{[n]} \\ r\end{array}\right)$ which sends $I=\left\{i_{1}<\cdots<i_{r}\right\}$ to

(1) $\left\{i_{1}-1<i_{2}-1<\cdots<i_{r}-1\right\}$ if $i_{1}>1$,

(2) $\left\{i_{2}-1<\cdots<i_{r}-1<n\right\}$ if $i_{1}=1$.

Informally, $\operatorname{Sh}(I)=I-1$ with 0 's replaced by $n$.

Let $\mathcal{I}=(d, r, D, n, I)$ be a Schubert state and let $p \in \mathrm{S}$. Define a new shifted Schubert state $\operatorname{Sh}(p)(\mathcal{I})=\left(d^{\prime}, r, D-1, n, J\right)$ where $d^{\prime}$ and $J$ are given as follows: 
$J^{q}=I^{q}$ if $q \in \mathrm{S}-\{p\}, J^{p}=\mathrm{Sh}\left(I^{p}\right)$ and

(a) if $i_{1}^{p}>1, d^{\prime}=d$,

(b) if $i_{1}^{p}=1, d^{\prime}=d-1$.

The following proposition is related to the "action" of $n$-th roots of unity on the quantum cohomology of the Grassmannian $\operatorname{Gr}(r, n)[2]$. The geometry of this action of the center of $\mathrm{SL}(n)$ appeared in [5] (where it was used to give a new geometric proof of Bertram's Pieri and Giambelli rules [7] for the quantum cohomology of Grassmannians).

Proposition 2.7. With notation as above,

(1) $\operatorname{dim} \mathcal{I}=\operatorname{dim} \operatorname{Sh}(p) \mathcal{I}$,

(2) $\mathcal{I}$ is non-null $\Leftrightarrow \operatorname{Sh}(p) \mathcal{I}$ is non-null,

(3) $\langle\mathcal{I}\rangle=\langle\operatorname{Sh}(p)(\mathcal{I})\rangle$.

\subsection{The main result.}

Theorem 2.8. Let $\mathcal{I}=(d, r, D, n, I)$ be a Schubert state such that $0<r<n$ and

$$
\operatorname{dim} \mathcal{I}=\operatorname{dim} \operatorname{Gr}\left(d, r, \mathcal{Z}_{D, n}\right)-\sum_{p \in \mathrm{S}} \operatorname{codim}\left(\omega_{I^{p}}\right) \geq 0 .
$$

The following are equivalent:

(A) $\mathcal{I}$ is non-null.

(B) For every non-null Schubert state of the form $\mathcal{K}=\left(d^{\prime}, r^{\prime}, d, r, K\right)$ with $0<$ $r^{\prime}<r$, the following inequality holds:

$$
-\chi\left(d^{\prime}, r^{\prime}, D-d, n-r\right)+\sum_{p \in \mathrm{S}} \sum_{a \in K^{p}}\left(n-r+a-i_{a}^{p}\right) \leq 0 .
$$

(C) For every Schubert state of the form $\mathcal{K}=\left(d^{\prime}, r^{\prime}, d, r, K\right)$ with $0<r^{\prime}<r$ and $\langle\mathcal{K}\rangle \neq 0$, inequality $\left(\dagger_{\mathcal{K}}^{\mathcal{I}}\right)$ holds.

(D) For every Schubert state of the form $\mathcal{K}=\left(d^{\prime}, r^{\prime}, d, r, K\right)$ with $0<r^{\prime}<r$ and $\langle\mathcal{K}\rangle=1$, inequality $\left(\dagger_{\mathcal{K}}^{\mathcal{I}}\right)$ holds.

Remark 2.9. For $p \in S$, let $\left(\lambda_{1}^{p} \geq \cdots \geq \lambda_{r}^{p}\right)=\lambda\left(I^{p}\right)$ (see equation (1.1)). Then inequality $\left(\dagger_{\mathcal{K}}^{\mathcal{I}}\right)$ takes the following form:

$$
\sum_{p \in S} \sum_{a \in K^{p}} \lambda_{a}^{p} \leq d^{\prime}(n-r)+(d-D) r^{\prime}+r^{\prime}(n-r) .
$$

Remark 2.10. The cases $r^{\prime}=0$ or $r^{\prime}=r$ are left out in the conditions (B), (C) and (D) of Theorem 2.8. The only non-null Schubert state $\mathcal{K}$ with $r^{\prime}=r$ is $(d, r, d, r, K)$ where $K^{p}=[r]$ for every $p \in \mathrm{S}$. In this case, inequality $\left(\dagger_{\mathcal{K}}^{\mathcal{I}}\right)$ is the same as the inequality (2.3). The only non-null Schubert state $\mathcal{K}$ with $r^{\prime}=0$ is $(0,0, d, r, K)$ where $K^{p}=\emptyset$ for every $p \in \mathrm{S}$. It is easy to see that the corresponding inequality $(\dagger \mathcal{I})$ is always (trivially) satisfied.

The implications $(B) \Rightarrow(C) \Rightarrow(D)$ are trivial. The implications $(A) \Rightarrow(B)$ and $(C) \Rightarrow(A)$ will be shown in Sections 4 and 7.1, respectively. In Section 8.4 we will show that (D) implies (B), using Proposition 10.1 if the characteristic of the base field is non-zero. 


\section{Applichtions of Theorem 2.8}

3.1. Proof of Theorem 1.3. Theorem 1.3 is a special case (when $D=0$ ) of

Proposition 3.1. Let $\mathcal{I}=(d, r, D, n, I)$ be a Schubert state such that $\operatorname{dim} \mathcal{I}=0$ and $0<r<n$. Then the following are equivalent:

(1) $\langle\mathcal{I}\rangle \neq 0$.

(2) There exist $A^{(1)}, \ldots, A^{(s)} \in \mathrm{SU}(r)$ such that

- $A^{(1)} A^{(2)} \cdots A^{(s)}=1$.

- $A^{(1)}$ is in the conjugacy class $\zeta_{r}^{d} \beta\left(I^{1}\right)$ and for $j \geq 2, A^{(j)}$ is in the conjugacy class $\beta\left(I^{j}\right)$.

Proof. By Lemma 2.6, $\langle\mathcal{I}\rangle \neq 0$ if and only if $\langle(d+r, r, D+n, n, I)\rangle \neq 0$, and clearly, $\zeta_{r}^{d}=\zeta_{r}^{d+r}$. We can therefore assume that $0 \leq d<r$ without loss of generality.

Let $I^{p_{1}}=\left\{i_{1}<\cdots<i_{r}\right\}$; we consider $\mathcal{J}=\operatorname{Sh}\left(p_{1}\right)^{i_{d}} \mathcal{I}=\left(0, r, D-i_{d}, n, J\right)$ (see Section 2.5). From Proposition 2.7, $\langle\mathcal{I}\rangle \neq 0$ if and only if $\langle\mathcal{J}\rangle \neq 0$.

It is easy to see that for $I \in\left(\begin{array}{c}{[n]} \\ r\end{array}\right)$,

- $\beta(\operatorname{Sh}(I))=\beta(I)$ if $i_{1} \neq 1$,

- $\beta(\operatorname{Sh}(I))=\zeta_{r} \beta(I)$ if $i_{1}=1$.

Hence, $\beta\left(J^{p_{1}}\right)=\zeta_{r}^{d} \beta\left(I^{p_{1}}\right)$ and $\beta\left(J^{p_{j}}\right)=\beta\left(I^{p_{j}}\right)$ for $j=2, \ldots, s$. Therefore if the proposition holds for $\mathcal{J}$, then it also holds for $\mathcal{I}$. Hence it suffices to prove the proposition assuming $d=0$.

Assume $d=0$. From the equivalence of $(\mathrm{A})$ and $(\mathrm{C})$ in Theorem 2.8, $\mathcal{I}$ is non-null if and only if a system of inequalities $\left(\dagger^{\mathcal{T}}\right)$ indexed by Schubert states of the form $\mathcal{K}=\left(d^{\prime}, r^{\prime}, 0, r, K\right)$ with $0<r^{\prime}<r$ such that $\langle\mathcal{K}\rangle \neq 0$ holds. Similarly, according to Theorem 1.1, (2) holds if and only if a system of inequalities indexed by the same set of $\mathcal{K}$ holds (see Remark 2.5).

Set $\left(\lambda_{1}^{j}, \ldots, \lambda_{r}^{j}\right)=\lambda\left(I^{p_{j}}\right)$ for $j=1, \ldots, s$. Then the inequality $\left(\dagger_{\mathcal{K}}^{\mathcal{I}}\right)$ (see Remark 2.9) is

$$
\sum_{j=1}^{s} \sum_{a \in K^{p^{j}}} \lambda_{a}^{j} \leq d^{\prime}(n-r)-D r^{\prime}+r^{\prime}(n-r) .
$$

Using the hypothesis $\operatorname{dim} \mathcal{I}=0$, we have

$$
\sum_{j=1}^{s} \sum_{a=1}^{r} \lambda_{a}^{j}=r(n-r)-D r .
$$

Subtract $r^{\prime}$ times equality (3.2) from $r$ times inequality (3.1) to obtain

$$
\sum_{j=1}^{s} \sum_{a \in K^{p_{j}}}\left(r \lambda_{a}^{j}-\sum_{b=1}^{r} \lambda_{b}^{j}\right) \leq d^{\prime} r(n-r) .
$$

Let $\beta\left(I^{p_{j}}\right)=\left(\alpha_{1}^{j}, \ldots, \alpha_{r}^{j}\right)$ for $j=1, \ldots, s$. Dividing inequality $(3.3)$ by $r(n-r)$, we see that inequality (3.1) is equivalent to the following inequality (see Definition 1.2):

$$
\sum_{j=1}^{s} \sum_{a \in K^{p_{j}}} \alpha_{a}^{j} \leq d^{\prime}
$$

The above inequality is the same as the one corresponding to Theorem 1.1 for $\mathcal{K}$ and the proposition is proved. 


\subsection{Proof of Theorem 1.5.}

Proposition 3.2. Let $\mathcal{I}=(d, r, 0, n, I)$ be a Schubert state where $0<r<n$. The following are equivalent:

(1) $\mathcal{I}$ is non-null.

(2) For some $c \leq d, q^{c}$ appears in the product $\omega_{I^{p_{1}}} \star \omega_{I^{p_{2}}} \star \cdots \star \omega_{I^{p_{s}}}$.

Proof. Assume (1). Let $\mathcal{W}=\mathcal{O}_{\mathbb{P}^{1}}^{\oplus n}$, and let $\mathcal{E} \in \mathrm{Fl}_{\mathrm{S}}(\mathcal{W})$ be a generic point. For $p \in S$, let $\pi_{p}: \operatorname{Gr}(d, r, \mathcal{W}) \rightarrow \operatorname{Gr}\left(r, \mathcal{W}_{p}\right)$ be the natural morphism. Let

$$
\Omega=\bigcap_{p \in \mathrm{S}} \pi_{p}^{-1}\left(\Omega_{I^{p}}^{o}\left(E_{\bullet}^{p}\right)\right) \subseteq \operatorname{Gr}(d, r, \mathcal{W}) .
$$

Since $\mathcal{I}$ is non-null, $\Omega$ is a non-empty proper intersection of dimension $\operatorname{dim} \mathcal{I}$. Pick a large collection of points $Q=\left\{q_{1}, \ldots, q_{m}\right\}$ such that the natural morphism

$$
\Gamma: \operatorname{Gr}(d, r, \mathcal{W}) \rightarrow \prod_{q \in Q} \operatorname{Gr}\left(r, \mathcal{W}_{q}\right)
$$

is quasi-finite (see e.g. [11], Lemma 7.4).

Let $G=\overline{\Gamma(\Omega)}$. Since $\mathcal{E}$ is generic, each irreducible component of $G$ is of dimension $\operatorname{dim} \mathcal{I}$. Clearly there exist $\omega_{L^{q}} \in H^{*}\left(\operatorname{Gr}\left(r, \mathcal{W}_{q}\right)\right)$ for $q \in Q$ such that $[G] \cdot\left(\prod_{q \in Q} \omega_{L^{q}}\right)$ is a non-zero multiple of the class of a point in $H^{2 m r(n-r)}\left(\prod_{q \in Q} \operatorname{Gr}\left(r, \mathcal{W}_{q}\right)\right)$.

Choose a generic point

$$
\left(E_{\bullet}^{q} \mid q \in Q\right) \in \prod_{q \in Q} \operatorname{Fl}\left(\mathcal{W}_{q}\right) .
$$

It is clear that $G$ meets $\prod_{q \in Q} \Omega_{L^{q}}\left(E_{\bullet}^{q}\right)$ in a non-empty finite set. Now, $\Gamma(\Omega) \cap$ $\left(\prod_{q \in Q} \Omega_{L^{q}}^{o}\left(E_{\bullet}^{q}\right)\right)$ is dense in $G \cap\left(\prod_{q \in Q} \Omega_{L^{q}}\left(E_{\bullet}^{q}\right)\right.$ ) (see e.g. [6], Proposition 1.1) and is therefore a non-empty finite set. Hence,

$$
\left\langle\omega_{I^{p_{1}}}, \ldots, \omega_{I^{p_{s}}}, \omega_{L^{q_{1}}}, \ldots, \omega_{L^{q_{m}}}\right\rangle_{d} \neq 0 .
$$

Using the associativity of the quantum product, we conclude that (2) holds.

Suppose (2) holds. According to Lemma 3.3, there exist $L^{1}, \ldots, L^{m} \in\left(\begin{array}{c}{[n]} \\ r\end{array}\right)$ (for some $m$ ) such that

$$
\left\langle\omega_{I^{p_{1}}}, \ldots, \omega_{I^{p_{s}}}, \omega_{L^{1}}, \ldots, \omega_{L^{m}}\right\rangle_{d} \neq 0 .
$$

Add $m$ new points $\left\{q_{1}, \ldots, q_{m}\right\} \in \mathbb{P}^{1}-\mathrm{S}$. Let $E_{\bullet}^{1}, \ldots, E_{\bullet}^{s+m}$ be generic flags on $\mathbb{C}^{n}$. From the non-vanishing of the Gromov-Witten number (3.4), there is a non-empty finite set of maps $f: \mathbb{P}^{1} \rightarrow \operatorname{Gr}(r, n)$ such that $f\left(p_{j}\right) \in \Omega_{I^{p_{j}}}^{o}\left(E_{\bullet}^{j}\right)$ for $j=1, \ldots, s$ and $f\left(q_{i}\right) \in \Omega_{L^{i}}^{o}\left(E_{\bullet}^{s+i}\right)$ for $i=1, \ldots, m$. Let $\mathcal{E} \in \mathrm{Fl}_{\mathrm{S}}(\mathcal{W})$ be the point whose $p_{j}$-th coordinate is $E^{j}$ (using the given isomorphism $\mathcal{W}_{p_{j}} \stackrel{\sim}{\rightarrow} \mathbb{C}^{n}$ ). The nonvanishing of the Gromov-Witten number (3.4) implies that $\bigcap_{p \in \mathrm{S}} \pi_{p}^{-1}\left(\Omega_{I^{p}}^{o}\left(E_{\bullet}^{p}\right)\right)$ is non-empty. Therefore $\mathcal{I}$ is non-null.

Lemma 3.3. For every cohomology class $\omega_{I}$ of a Schubert variety in $\operatorname{Gr}(r, n)$ and every integer $e>0$, there exist $L^{1}, \ldots, L^{m} \in\left(\begin{array}{c}{[n]} \\ r\end{array}\right)$ such that $q^{e}$ appears in the quantum product $\omega_{I} \star \omega_{L^{1}} \star \cdots \star \omega_{L^{m}}$.

Proof. It suffices by induction to consider only the case $e=1$. Multiply by the dual $\omega_{I^{\prime}}$ of $\omega_{I}$ to reduce to the case of $I=[r]$ (so that $\omega_{I}$ is the class of a point). But this case follows from Bertram's Pieri formula [7], by multiplying $\omega_{I}$ with the class of a codimension 1 Schubert variety: $\omega_{[r]} \star \omega_{\{n-r, n-r+2, n-r+3, \ldots, n\}}=q \omega_{\{2,3, \ldots, r, n\}}$. 
3.2.1. Proof of Theorem 1.5. Let $\mathcal{I}=(d, r, 0, n, I)$ where $I^{p_{j}}=I^{j}$ for $j=1, \ldots, s$. By Proposition 3.2, the condition in (a) is that $\mathcal{I}$ is non-null. Let $\mathcal{L}=\operatorname{Sh}\left(p_{1}\right)^{q n+i_{b}^{1}} \mathcal{I}$ $=\left(0, r,-\left(q n+i_{b}^{1}\right), n, L\right)$ for some $L$. By Lemma 2.6 and Proposition 2.7, $\operatorname{dim} \mathcal{I}=$ $\operatorname{dim} \mathcal{L}$ and $\mathcal{I}$ is non-null if and only if $\mathcal{L}$ is non-null. Condition (b) is just the system of inequalities obtained from Theorem 2.8 for $\mathcal{L}$ to be non-null. If equality holds in inequality (1.3), then any term of the form $q^{c} \omega_{J}$ in (a) is necessarily a non-zero multiple of $q^{d} \omega_{[r]}$. This shows the equivalence of (c) with the other conditions.

3.3. Saturation in the fusion ring. We will first formulate the explicit relation between structure coefficients in the fusion ring of $\mathrm{SU}(r)$ at level $k$ and GromovWitten numbers of Grassmannians $\operatorname{Gr}(r, n)$ where $n=r+k$.

Proposition 3.4. Let $\mathcal{I}=(d, r, D, n, I)$ be a Schubert state with $\operatorname{dim} \mathcal{I}=0$ and $d=0$. Set $\mu^{j}=\lambda\left(I^{p_{j}}\right)$ for $j=1, \ldots, s$. Then

$$
\langle\mathcal{I}\rangle=N_{0}^{(k)}\left(\bar{\mu}^{1}, \ldots, \bar{\mu}^{s}\right) .
$$

In Section 3.5, we will deduce Proposition 3.4 from a theorem of Witten [33].

3.3.1. Fusion structure coefficients and the multiplicative eigenvalue problem. As in Section 1.5, let $P_{k}$ be the set of irreducible representations $\bar{\mu}$ of $\mathrm{SU}(r)$ such that $\mu_{1}-\mu_{r} \leq k$. For a representation $\bar{\mu} \in P_{k}$, define a conjugacy class $\alpha(\bar{\mu}, k)$ of $\operatorname{SU}(r)$ by the following formula:

$$
\alpha(\bar{\mu}, k)=\frac{1}{k}\left(\mu-\frac{|\mu|}{r}(1, \ldots, 1)\right) .
$$

Proposition 3.5. Let $\bar{\mu}^{1}, \ldots, \bar{\mu}^{s} \in P_{k}$ be such that $\sum_{j=1}^{s}\left|\bar{\mu}^{j}\right|=0 \in \mathbb{Z} / r \mathbb{Z}$. The following are equivalent:

(1) $N_{0}^{(k)}\left(\bar{\mu}^{1}, \ldots, \bar{\mu}^{s}\right) \neq 0$.

(2) There exist $A^{(1)}, \ldots, A^{(s)} \in \mathrm{SU}(r)$ with $A^{(j)}$ in the conjugacy class $\alpha\left(\bar{\mu}^{j}, k\right)$ such that $A^{(1)} A^{(2)} \cdots A^{(s)}=1$.

Proof. Let $n=r+k$. Find $I^{1}, \ldots, I^{s} \in\left(\begin{array}{c}{[n]} \\ r\end{array}\right)$ such that $\bar{\lambda}\left(I^{j}\right)=\bar{\mu}^{j}$ for $j=1, \ldots, s$. Since $\sum_{j=1}^{s}\left|\bar{\mu}^{j}\right|=0 \in \mathbb{Z} / r \mathbb{Z}$, there exists a possibly negative integer $D$ such that

$$
\sum_{j=1}^{s}\left|\lambda\left(I^{j}\right)\right|=r(n-r)-D r
$$

Let $I^{p_{j}}=I^{j}$ for $j=1, \ldots, s$, and $\mathcal{I}=(0, r, D, n, I)$. Using Proposition 2.2 and equation (3.6),

$$
\operatorname{dim} \mathcal{I}=\operatorname{dim} \operatorname{Gr}\left(0, r, \mathcal{Z}_{D, n}\right)-\sum_{j=1}^{s} \operatorname{codim} \omega_{I^{j}}=r(n-r)-D r-\sum_{j=1}^{s}\left|\lambda\left(I^{j}\right)\right|=0 .
$$

By Proposition 3.4, $\langle\mathcal{I}\rangle=N_{0}^{(k)}\left(\bar{\mu}^{1}, \ldots, \bar{\mu}^{s}\right)$. It is easy to see that the conjugacy class $\alpha\left(\bar{\mu}_{j}, k\right)$ is the same as $\beta\left(I^{j}\right)$. We can now conclude the proof by using Proposition 3.1.

3.3.2. Proof of Theorem 1.8. It is easy to see by direct inspection that $\alpha(M \bar{\mu}, M k)$ $=\alpha(\bar{\mu}, k)$ for any positive integer $M$. Therefore by Proposition $3.5, N_{0}^{(k)}\left(\bar{\mu}^{1}, \ldots, \bar{\mu}^{s}\right)$ $\neq 0$ if and only if $N_{0}^{(M k)}\left(M \bar{\mu}^{1}, \ldots, M \bar{\mu}^{s}\right) \neq 0$. This concludes the proof of Theorem 1.8 . 
3.4. Fusion rings and the quantum cohomology of Grassmannians. It is known classically that there is a ring-theoretic surjection from the polynomial representation ring of $\mathrm{U}(r)$ to the cohomology $H^{*}(\operatorname{Gr}(r, n), \mathbb{Z})$ of the Grassmannian $\operatorname{Gr}(r, n)$ (see e.g. [6], Section 7). Although the representation theory of $\mathrm{U}(r)$ is closely related to that of $\mathrm{SU}(r)$ ("twisted" by the representation theory of $\mathrm{U}(1)$ ), only the representation ring of $\mathrm{U}(r)$ relates to the cohomology of Grassmannians in a ring-theoretic framework.

This phenomenon continues in the quantum setting. The fusion rings are the quantum analogues of representation rings. We have three fusion rings in this picture (for more details see [33]):

(1) the fusion ring of $\mathrm{SU}(r)$ at level $k$, denoted as in Section 1.5 by $R(\mathrm{SU}(r))_{k}$,

(2) the fusion ring $R(\mathrm{U}(1))_{n r}$ of $\mathrm{U}(1)$ at level $n r$ which is identified with $\mathbb{Z}[x] /\left(x^{n r}-1\right)$,

(3) the fusion ring $R(\mathrm{U}(r))_{k, n}$ of $\mathrm{U}(r)$ at $\mathrm{SU}(r)$ level $k$ and $\mathrm{U}(1)$ level $n(=r+$ $k$ ). Recall ([33], [1]) that $R(\mathrm{U}(r))_{k, n}$ is additively generated by weakly decreasing sequences of non-negative integers: $\lambda=\left(\lambda_{1} \geq \lambda_{2} \geq \cdots \geq \lambda_{r}\right) \in$ $\mathbb{Z}_{\geq 0}^{r}$ such that $\lambda_{1} \leq k$.

In analogy with the classical situation, $R(\mathrm{U}(r))_{k, n}$ is directly related to quantum cohomology by the following theorem of Witten [33].

Theorem 3.6. The natural additive map

$$
W: \mathrm{QH}^{*}(G r(r, n)) /(q-1) \rightarrow R(\mathrm{U}(r))_{k, n}
$$

given by $W\left(\omega_{I}\right)=\lambda(I)$ is an isomorphism of rings.

The fusion ring $R(\mathrm{U}(r))_{k, n}$ is a "twisted" form of $R(\mathrm{SU}(r))_{k}$. We will now make this relation precise (for more details see [33], page 414, and [15], pages 11, 12). Inside $R(\mathrm{SU}(r))_{k} \otimes_{\mathbb{C}} R(\mathrm{U}(1))_{n r}$ consider the abelian subgroup $\widetilde{R}$ spanned by $\bar{\lambda} \otimes x^{a}$ such that $|\bar{\lambda}| \equiv a(\bmod r)$. It is immediate that $\widetilde{R}$ is a unital subring of $R(\mathrm{SU}(r))_{k} \otimes_{\mathbb{Z}} R(\mathrm{U}(1))_{n r}$.

Consider the following operator acting on $\widetilde{R}$ :

$$
T\left(\bar{\lambda} \otimes x^{a}\right)=\bar{\eta} \otimes x^{n+a}
$$

where $\bar{\eta}$ is related to $\bar{\lambda}$ by the cyclic shift $\eta=\left(k+\lambda_{r} \geq \lambda_{1} \geq \lambda_{2} \geq \cdots \geq \lambda_{r-1}\right)$ and $a \equiv|\bar{\lambda}|(\bmod r)$. It is easy to check that $T(u)=u \cdot\left((k, 0, \ldots, 0) \otimes x^{n}\right)$. The $\mathbb{Z}$-submodule of $\widetilde{R}$ generated by elements $T(u)-u$ for $u \in \widetilde{R}$ is an ideal $I$ of $\widetilde{R}$.

Lemma 3.7. (1) Each orbit $\left\{T^{b}\left(\bar{\mu} \otimes x^{a}\right)_{b=1}^{r}\right\}$ where $a \equiv|\bar{\mu}|(\bmod r)$, contains a unique element of the form $\bar{\lambda} \otimes x^{|\lambda|} \in \widetilde{R}$ where the sequence $\lambda$ satisfies $\lambda_{1} \leq k$ and $\lambda_{r} \geq 0$.

(2) If $\bar{\mu}=0$, then the $T$ orbit of $\bar{\mu} \otimes 1$ does not contain any element of the form $\bar{\nu} \otimes 1$ with $\bar{\nu} \neq 0$.

Proof. Let $K \in\left(\begin{array}{c}{[n]} \\ r\end{array}\right)$ be such that $\bar{\lambda}(K)=\bar{\mu}$ and set $c=a-|\lambda|$. Clearly, $\bar{\mu} \otimes x^{a}=$ $\bar{\lambda}(K) \otimes x^{c+|\lambda(K)|}$. Let $J=\mathrm{Sh}^{-1}(K)$ (see Section 2.5). That is, $J$ is obtained by adding 1 to elements of $K$ and replacing $n+1$ 's by 1 .

It is easy to see that $\bar{\lambda}(J) \otimes x^{c+|\lambda(J)|+r}$ equals $\bar{\lambda}(K) \otimes x^{c+|\lambda(K)|}$ if $n \notin K$ and it equals $T\left(\bar{\lambda}(K) \otimes x^{c+|\lambda(K)|}\right)$ if $n \in K$. Now by assumption $c \equiv 0(\bmod r)$ and hence there is a unique $\ell \in[n]$ such that $c+\ell r \equiv 0(\bmod n r)$. We repeat this process $\ell$ 
times. The sought for $\lambda$ is $\lambda\left(\mathrm{Sh}^{-\ell}(K)\right)$. This proves (1). Assertion (2) follows from the definition of $T$.

The linear map $\widetilde{R} \rightarrow R(\mathrm{U}(r))_{k, n}$ sending $\bar{\lambda} \otimes x^{|\lambda|} \in \widetilde{R}$ to $\lambda \in R(\mathrm{U}(r))_{k, n}$ is a ring homomorphism with kernel $I$ and induces an isomorphism of rings (see [33], page 416)

$$
\widetilde{R} / I \stackrel{\sim}{\rightarrow} R(\mathrm{U}(r))_{k, n}
$$

Let $K=\{1, k+2, k+3, \ldots, k+r=n\} \subset[n]$. Then using equation (3.8) on $\lambda=(0,0, \ldots, 0) \in \mathbb{Z}^{r}$, we obtain the following equality in $R(\mathrm{U}(r))_{k, n}$ (note that $\left.1 \otimes x^{r} \in \widetilde{R}\right)$ :

$$
W\left(\omega_{K}\right)\left(1 \otimes x^{r}\right)=1 .
$$

Remark 3.8. For the purposes of this paper, we can take $\widetilde{R} / I$ to be the definition of $R(\mathrm{U}(r))_{k, n}$. Since Pieri's rule holds on both sides of equation (3.7) (using [7] on the quantum cohomology side and [14] for the fusion ring of $\mathrm{SU}(r)$ at level $k$ ), we can then conclude that $W$ is a ring isomorphism.

3.5. Proof of Proposition 3.4. We will first consider the case $D \leq 0$. Let $K=$ $\{1, k+2, k+3, \ldots, n\}$. We claim

$$
\langle\mathcal{I}\rangle=\left\langle\omega_{I^{1}}, \omega_{I^{2}}, \ldots, \omega_{I^{s}}, \omega_{K}, \omega_{K}, \ldots, \omega_{K}\right\rangle_{-D}
$$

with $\omega_{K}$ repeated $-D$ times (the right hand side is a usual Gromov-Witten number). To see this, apply the shift operation from Proposition 2.7 to each of the $-D$ $\omega_{K}$ 's appearing on the right hand side of equation (3.10) (see Remark 3.9).

According to Theorem 3.6, the right hand side of equation (3.10) equals the coefficient of $W\left(\omega_{[r]}\right)$ in the product

$$
W\left(\omega_{K}\right)^{-D} \prod_{j=1}^{s} W\left(\omega_{I^{j}}\right) \in R(\mathrm{U}(r))_{k, n} .
$$

Using equation (3.9), we note $W\left(\omega_{K}\right)=1 \otimes x^{n r-r}$. Also $W\left(\omega_{I^{j}}\right)=\bar{\lambda}\left(I^{j}\right) \otimes$ $x^{\operatorname{codim}\left(\omega_{I^{j}}\right)}$. Then using our assumption that $\operatorname{dim} \mathcal{I}=0$, the $\mathrm{U}(1)$ component of the product in $\widetilde{R}$ corresponds to $x$ raised to the number $(\bmod n r)$

$$
\sum_{j} \operatorname{codim}\left(I^{j}\right)+D r=r(n-r) .
$$

Thus, using Lemma $3.7(2)$, the coefficient of $W\left(\omega_{[r]}\right)$ in $W\left(\omega_{K}\right)^{-D} \prod_{j=1}^{s} W\left(\omega_{I^{j}}\right)$ equals the coefficient of the identity representation in the product $\prod_{j=1}^{s} \bar{\lambda}\left(I^{j}\right)$ in $R(\mathrm{SU}(r))_{k}$, which equals the right hand side of equation (3.5) as desired.

If $D>0$, let $L=\{k, k+1, \ldots, n-1\} \subseteq[n]$. It is easy to show, using Proposition 2.7 that

$$
\langle\mathcal{I}\rangle=\left\langle\omega_{I^{1}}, \omega_{I^{2}}, \ldots, \omega_{I^{s}}, \omega_{L}, \omega_{L}, \ldots, \omega_{L}\right\rangle_{0}
$$

with $\omega_{L}$ repeated $D$ times (the right hand side is now a classical cohomology structure coefficient). It is easy to check that $W\left(\omega_{L}\right)=1 \otimes x^{r}$ and we proceed as before.

Remark 3.9. Let $\mathcal{I}=(d, r, D, n, I)$ be a Schubert state, and suppose $q \in S$ and $I^{q}=\{n-r+1, n-r+2, \ldots, n\}$. Set $S^{\prime}=S-\{q\}$ and $\mathcal{I}^{\prime}=\left(d, r, D, n, I \mid S^{\prime}\right)$. Then $\left\langle\mathcal{I}^{\prime}\right\rangle=\langle\mathcal{I}\rangle$. 


\section{Proof of $(\mathrm{A}) \Rightarrow(\mathrm{B})$ In Theorem 2.8}

Definition 4.1. Let $\mathcal{I}=(d, r, D, n, I)$ be a Schubert state, $\mathcal{W}$ a vector bundle on $\mathbb{P}^{1}$ of degree $-D$ and rank $n$, and $\mathcal{E} \in \mathrm{Fl}_{\mathrm{S}}(\mathcal{W})$. For $p \in \mathrm{S}$, let $\pi_{p}: \operatorname{Gr}(d, r, \mathcal{W}) \rightarrow$ $\operatorname{Gr}\left(r, \mathcal{W}_{p}\right)$ be the natural morphism. Define the scheme-theoretic intersection

$$
\Omega^{o}(\mathcal{I}, \mathcal{W}, \mathcal{E})=\bigcap_{p \in \mathrm{S}} \pi_{p}^{-1}\left(\Omega_{I^{p}}^{o}\left(E_{\bullet}^{p}\right)\right) \subseteq \operatorname{Gr}(d, r, \mathcal{W}) .
$$

Definition 4.2. Let $\mathcal{I}=(d, r, D, n, I)$ and $\mathcal{K}=\left(d^{\prime}, r^{\prime}, d, r, K\right)$ be Schubert states. Define $\mathcal{I}_{\mathcal{K}}=\left(d^{\prime}, r^{\prime}, D, n, L\right)$ where $L^{p}=\left\{i_{a}^{p} \mid a \in K^{p}\right\}$ for $p \in S$.

Remark 4.3. Suppose $\mathcal{V} \in \Omega^{\circ}(\mathcal{I}, \mathcal{W}, \mathcal{E})$. By an easy computation at each $p \in S$ (see [13], Lemma 2 (i)) it follows that

$$
\Omega^{o}(\mathcal{K}, \mathcal{V}, \mathcal{E}(\mathcal{V})) \subseteq \Omega^{o}\left(\mathcal{I}_{\mathcal{K}}, \mathcal{W}, \mathcal{E}\right)
$$

under the inclusion $\operatorname{Gr}\left(d^{\prime}, r^{\prime}, \mathcal{V}\right) \subseteq \operatorname{Gr}\left(d^{\prime}, r^{\prime}, \mathcal{W}\right)$.

Lemma 4.4. Each irreducible component of $\Omega^{\circ}(\mathcal{I}, \mathcal{W}, \mathcal{E})$ is of dimension at least $\operatorname{dim} \mathcal{I}$.

Proof. Each irreducible component of $\operatorname{Gr}(d, r, \mathcal{W})$ passing through a point $\mathcal{V}$ is of dimension at least $\chi(\mathcal{H o m}(\mathcal{V}, \mathcal{W} / \mathcal{V})$ ) (see e.g. [21], Theorem I.5.17). Now, Schubert cells in a Grassmannian are smooth and are hence local complete intersections. By Krull's principal ideal theorem it is now easy to see that each irreducible component of $\Omega^{\circ}(\mathcal{I}, \mathcal{W}, \mathcal{E})$ passing through $\mathcal{V}$ is of dimension at least $\chi(\mathcal{H o m}(\mathcal{V}, \mathcal{W} / \mathcal{V}))-\sum_{p \in \mathrm{S}} \operatorname{codim}\left(\omega_{I^{p}}\right)$ as desired.

The proof of the following lemma will be given in Section 4.2.

Lemma 4.5. Let $\mathcal{W}=\mathcal{Z}_{D, n}$, and suppose $\mathcal{I}=(d, r, D, n, I)$ and $\mathcal{K}=\left(d^{\prime}, r^{\prime}, d, r, K\right)$ are non-null Schubert states. Then for generic $\mathcal{E} \in \mathrm{Fl}_{\mathrm{S}}(\mathcal{W})$, there exists an open dense subset of $\Omega^{\circ}(\mathcal{I}, \mathcal{W}, \mathcal{E})$ such that for $\mathcal{V}$ in this open dense subset,

(i) $\mathcal{V}$ and $\mathcal{W} / \mathcal{V}$ are evenly split vector bundles,

(ii) $\Omega^{o}(\mathcal{K}, \mathcal{V}, \mathcal{E}(\mathcal{V}))$ is a non-empty proper intersection.

Suppose $\mathcal{I}=(d, r, D, n, I)$ is a non-null Schubert state with $0<r<n$. Let $\mathcal{K}$ be a non-null Schubert state of the form $\left(d^{\prime}, r^{\prime}, d, r, K\right)$ with $0<r^{\prime}<r$. We will now show that inequality $(\dagger \mathcal{I})$ from Theorem 2.8 holds:

Let $\mathcal{E} \in \mathrm{Fl}_{\mathrm{S}}(\mathcal{W})$ be a generic point. Choose $\mathcal{V} \in \Omega^{\circ}(\mathcal{I}, \mathcal{W}, \mathcal{E})$ which satisfies conditions (i) and (ii) of Lemma 4.5. Now, $\mathcal{K}$ is non-null, and therefore by the choice of $\mathcal{V}$, the intersection $\Omega^{\circ}(\mathcal{K}, \mathcal{V}, \mathcal{E}(\mathcal{V}))$ is non-empty and each irreducible component is of dimension $\operatorname{dim} \mathcal{K}$. Since $\mathcal{E}$ is generic, each irreducible component of $\Omega^{o}\left(\mathcal{I}_{\mathcal{K}}, \mathcal{W}, \mathcal{E}\right)$ is of dimension $\operatorname{dim} \mathcal{I}_{\mathcal{K}}$. Therefore from inclusion (4.1), we obtain the inequality $\operatorname{dim} \mathcal{K}-\operatorname{dim} \mathcal{I}_{\mathcal{K}} \leq 0$. But $\operatorname{dim} \mathcal{K}-\operatorname{dim} \mathcal{I}_{\mathcal{K}}$ equals

$$
\begin{aligned}
& \left(\operatorname{dim} \operatorname{Gr}\left(d^{\prime}, r^{\prime}, \mathcal{Z}_{d, r}\right)-\operatorname{dim} \operatorname{Gr}\left(d^{\prime}, r^{\prime}, \mathcal{Z}_{D, n}\right)\right) \\
& +\left(\sum_{p \in \mathrm{S}} \sum_{b=1}^{r^{\prime}}\left(n-r^{\prime}+b-i_{k_{b}^{p}}^{p}-\left(r-r^{\prime}+b-k_{b}^{p}\right)\right)\right) \\
& \quad=-\chi\left(d^{\prime}, r^{\prime}, D-d, n-r\right)+\sum_{p \in \mathrm{S}} \sum_{a \in K^{p}}\left(n-r+a-i_{a}^{p}\right) .
\end{aligned}
$$

Therefore inequality $\left(\dagger_{\mathcal{K}}^{\mathcal{I}}\right)$ holds. 
Remark 4.6. From the above calculation, we see that $\operatorname{dim} \mathcal{K}-\operatorname{dim} \mathcal{I}_{\mathcal{K}}$ is the same as the left hand side of the inequality $\left(\dagger_{\mathcal{K}}^{\mathcal{I}}\right)$.

4.1. Universal families. Let $\mathcal{I}=(d, r, D, n, I)$ be a Schubert state and $\mathcal{W}$ a vector bundle of degree $-D$ and rank $n$ on $\mathbb{P}^{1}$.

Definition 4.7. Let $\mathcal{U}(\mathcal{I}, \mathcal{W})$ be the scheme consisting of pairs $(\mathcal{V}, \mathcal{E})$ where $\mathcal{E} \in$ $\mathrm{Fl}_{\mathrm{S}}(\mathcal{W})$ and $\mathcal{V} \in \Omega^{o}(\mathcal{I}, \mathcal{W}, \mathcal{E})$.

The rigorous scheme-theoretic definition of $\mathcal{U}(\mathcal{I}, \mathcal{W})$, as well as the proof of the following basic result, will be given in Section A.1.

Proposition 4.8. Let $\widehat{\mathcal{V}}$ be the universal subbundle on $\mathbb{P}^{1} \times \operatorname{Gr}(d, r, \mathcal{W})$.

(1) The natural morphism $\mathcal{U}(\mathcal{I}, \mathcal{W}) \rightarrow \mathrm{Fl}_{\mathrm{S}}(\widehat{\mathcal{V}})$ which takes $(\mathcal{V}, \mathcal{E})$ to $(\mathcal{V}, \mathcal{E}(\mathcal{V}))$ is smooth and surjective with connected fibres (see Section 2.2 for the definition of $\left.\mathrm{Fl}_{\mathrm{S}}(\widehat{\mathcal{V}})\right)$.

(2) If $\mathcal{W}$ is evenly split, then $\mathcal{U}(\mathcal{I}, \mathcal{W})$ is a smooth and connected scheme over the base field.

4.2. Proof of Lemma 4.5. Use notation from the statement of Lemma 4.5. Let $U(\mathcal{K})$ be the largest open subset of $\mathrm{Fl}_{\mathrm{S}}\left(\mathcal{Z}_{d, r}\right)$ such that for any $\mathcal{F} \in U(\mathcal{K})$, $\Omega^{o}\left(\mathcal{K}, \mathcal{Z}_{d, r}, \mathcal{F}\right)$ is a non-empty proper intersection. Since $\mathcal{K}$ is assumed to be nonnull, $U(\mathcal{K})$ is non-empty. It is clear that $U(\mathcal{K})$ is invariant under automorphisms of $\mathcal{Z}_{d, r}$.

By Proposition 4.8, if we let $\widehat{\mathcal{V}}$ be the universal subbundle on $\mathbb{P}^{1} \times \operatorname{Gr}(d, r, \mathcal{W})$, there are surjective morphisms

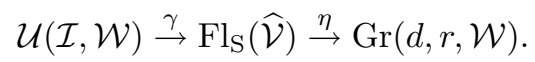

Since $\mathcal{I}$ is non-null, $\operatorname{Gr}(d, r, \mathcal{W})$ is non-empty. By Proposition 2.2, the subset $U_{E S}$ of points in $\operatorname{Gr}(d, r, \mathcal{W})$ where the subbundle and the quotient bundle are evenly split is non-empty, open and dense. Consider the subset $\widehat{U}$ of $\eta^{-1}\left(U_{E S}\right)$ consisting of points $(\mathcal{V}, \mathcal{F})$ so that " $\mathcal{F} \in U(\mathcal{K})$ ". This definition makes sense because if $(\mathcal{V}, \mathcal{F}) \in$ $\eta^{-1}\left(U_{E S}\right)$, then $\mathcal{V}$ is evenly split and $U(\mathcal{K})$ is invariant under automorphisms of $\mathcal{Z}_{d, r}$. The openness of $U(\mathcal{K}) \subseteq \mathrm{Fl}_{\mathrm{S}}\left(\mathcal{Z}_{d, r}\right)$ implies that $\widehat{U} \subseteq \mathrm{Fl}_{\mathrm{S}}(\widehat{\mathcal{V}})$ is open. For $\mathcal{V} \in U_{E S} \subseteq$ $\operatorname{Gr}(d, r, \mathcal{W})$, there exists $\mathcal{F} \in \mathrm{Fl}_{\mathrm{S}}(\mathcal{V})$ such that $(\mathcal{V}, \mathcal{F}) \in \widehat{U}(U(\mathcal{K})$ is non-empty), and therefore $\widehat{U} \neq \emptyset$. Consider the non-empty open subset $\gamma^{-1}(\widehat{U}) \subseteq \mathcal{U}(\mathcal{I}, \mathcal{W})$. Using Lemma 4.9 we see that for generic $\mathcal{E} \in \mathrm{Fl}_{\mathrm{S}}(\mathcal{W}), \Omega^{o}(\mathcal{I}, \mathcal{W}, \mathcal{E}) \cap \gamma^{-1}(\widehat{U})$ is dense in $\Omega^{\circ}(\mathcal{I}, \mathcal{W}, \mathcal{E})$, and this proves Lemma 4.5.

See Lemma 8.4 of [6] for the proof of the following statement.

Lemma 4.9. Let $f: X \rightarrow Y$ be a morphism of irreducible schemes and $U_{X} a$ non-empty open subset of $X$. Then there exists a non-empty open subset $U_{Y}$ of $Y$ such that for $y \in U_{Y}, U_{X} \cap f^{-1}(y)$ is dense in $f^{-1}(y)$.

We note the following corollary to Lemma 4.5:

Corollary 4.10. Suppose that $\mathcal{I}=(d, r, D, n, I)$ and $\mathcal{K}=\left(d^{\prime}, r^{\prime}, d, r, K\right)$ are nonnull Schubert states. Then $\mathcal{I}_{\mathcal{K}}$ is non-null.

Proof. Let $\mathcal{W}=\mathcal{Z}_{D, n}$. By Lemma 4.5, for generic $\mathcal{E} \in \mathrm{Fl}_{\mathrm{S}}(\mathcal{W})$, there exists $\mathcal{V} \in \Omega^{o}(\mathcal{I}, \mathcal{W}, \mathcal{E})$ such that $\Omega^{o}(\mathcal{K}, \mathcal{V}, \mathcal{E}(\mathcal{V})) \neq \emptyset$. Using the inclusion Remark 4.3, $\Omega^{o}\left(\mathcal{I}_{\mathcal{K}}, \mathcal{W}, \mathcal{E}\right) \neq \emptyset$ and since $\mathcal{E} \in \mathrm{Fl}_{\mathrm{S}}(\mathcal{W})$ is generic, $\mathcal{I}_{\mathcal{K}}$ is non-null. 


\section{TANGent SPACES}

In this section we denote the Zariski tangent space of a scheme $X$ at a point $x \in X$ by $T(X)_{x}$. See Section 2.7 of [29] for the following description of the tangent space of a Schubert variety:

Lemma 5.1. Let $I=\left\{i_{1}<\cdots<i_{r}\right\} \in\left(\begin{array}{c}{[n]} \\ r\end{array}\right)$ and let $W$ be a vector space of dimension $n$. Let $E_{\bullet}$ be a complete flag on $W$ and $V \in \Omega_{I}^{o}\left(E_{\bullet}\right)$. Let $E_{\bullet}(V)$ and $E_{\bullet}(W / V)$ denote the induced flags on $V$ and $W / V$, respectively. Then

$$
T\left(\Omega_{I}^{o}\left(E_{\bullet}\right)\right)_{V} \subseteq T(\operatorname{Gr}(r, W))_{V}=\operatorname{Hom}(V, W / V)
$$

is given by

$$
T\left(\Omega_{I}^{o}\left(E_{\bullet}\right)\right)_{V}=\left\{\phi \in \operatorname{Hom}(V, W / V) \mid \phi\left(E_{a}(V)\right) \subseteq E_{i_{a}-a}(W / V) \text { for } a \in[r]\right\} .
$$

Lemma 5.2. Let $\mathcal{I}$ be a Schubert state of the form $(d, r, D, n, I), \mathcal{W}$ a vector bundle of degree $-D$ and rank $n$ on $\mathbb{P}^{1}, \mathcal{E} \in \mathrm{Fl}_{\mathrm{S}}(\mathcal{W})$, and $\mathcal{V} \in \Omega^{\circ}(\mathcal{I}, \mathcal{W}, \mathcal{E})$. Then setting $\mathcal{Q}=\mathcal{W} / \mathcal{V}$

$$
T\left(\Omega^{o}(\mathcal{I}, \mathcal{W}, \mathcal{E})\right)_{\mathcal{V}}=\left\{\phi \in \operatorname{Hom}(\mathcal{V}, \mathcal{Q}) \mid \phi_{p}\left(E_{a}^{p}(\mathcal{V})\right) \subseteq E_{i_{a}^{p}-a}^{p}(\mathcal{Q}) \text { for } p \in \mathrm{S}, a \in[r]\right\}
$$

Proof. Let $\mathcal{E}=\left(E_{\bullet}^{p} \mid p \in \mathrm{S}\right) \in \mathrm{Fl}_{\mathrm{S}}(\mathcal{W})$. The tangent space of $\operatorname{Gr}(r, d, \mathcal{W})$ at the point corresponding to $\mathcal{V}$ is $\operatorname{Hom}(\mathcal{V}, \mathcal{Q})$ (see e.g. [23], Theorem 8.2.1). The tangent space of $\Omega_{I^{p}}^{o}\left(E_{\bullet}^{p}\right)$ in $G r\left(r, \mathcal{W}_{p}\right)$ is described by Lemma 5.1. The lemma now follows from the scheme-theoretic description of $\Omega^{\circ}(\mathcal{I}, \mathcal{W}, \mathcal{E})$ in Definition 4.1.

Lemma 5.2 motivates the following definition.

Definition 5.3. Let $\mathcal{I}=(d, r, D, n, I)$ be a Schubert state, $\mathcal{V}$ a vector bundle on $\mathbb{P}^{1}$ of degree $-d$ and rank $r, \mathcal{Q}$ a vector bundle on $\mathbb{P}^{1}$ of degree $d-D$ and rank $n-r, \mathcal{F} \in \mathrm{Fl}_{\mathrm{S}}(\mathcal{V})$ and $\mathcal{G} \in \mathrm{Fl}_{\mathrm{S}}(\mathcal{Q})$. Define

$$
\operatorname{Hom}_{\mathcal{I}}(\mathcal{V}, \mathcal{Q}, \mathcal{F}, \mathcal{G})=\left\{\phi \in \operatorname{Hom}(\mathcal{V}, \mathcal{Q}) \mid \phi_{p}\left(F_{a}^{p}\right) \subseteq G_{i_{a}^{p}-a}^{p} \text { for } p \in \mathrm{S}, a \in[r]\right\}
$$

Lemma 5.4. Using the notation of Definition 5.3,

(1) for each $(\mathcal{F}, \mathcal{G}) \in \mathrm{Fl}_{\mathrm{S}}(\mathcal{V}) \times \mathrm{Fl}_{\mathrm{S}}(\mathcal{Q})$,

$$
\operatorname{dim} \operatorname{Hom}_{\mathcal{I}}(\mathcal{V}, \mathcal{Q}, \mathcal{F}, \mathcal{G}) \geq \operatorname{dim} \mathcal{I}+h^{1}\left(\mathbb{P}^{1}, \mathcal{H o m}(\mathcal{V}, \mathcal{Q})\right)
$$

(2) the set of points $(\mathcal{F}, \mathcal{G}) \in \mathrm{Fl}_{\mathrm{S}}(\mathcal{V}) \times \mathrm{Fl}_{\mathrm{S}}(\mathcal{Q})$ for which equality holds in (1) is open (possibly empty),

(3) if there exists $(\mathcal{F}, \mathcal{G}) \in \mathrm{Fl}_{\mathrm{S}}(\mathcal{V}) \times \mathrm{Fl}_{\mathrm{S}}(\mathcal{Q})$ such that $\operatorname{dim} \operatorname{Hom}_{\mathcal{I}}(\mathcal{V}, \mathcal{Q}, \mathcal{F}, \mathcal{G}) \leq$ $\operatorname{dim} \mathcal{I}$, then $h^{1}\left(\mathbb{P}^{1}, \mathcal{H o m}(\mathcal{V}, \mathcal{Q})\right)=0$ and the open set in $(2)$ is non-empty.

Proof. For each $p \in \mathrm{S}$, consider $\pi_{p}: \operatorname{Hom}(\mathcal{V}, \mathcal{Q}) \rightarrow \operatorname{Hom}\left(\mathcal{V}_{p}, \mathcal{Q}_{p}\right)$ and define

$$
B_{p}=\left\{\psi \in \operatorname{Hom}\left(\mathcal{V}_{p}, \mathcal{Q}_{p}\right) \mid \psi\left(F_{a}^{p}\right) \subseteq G_{i_{a}^{p}-a}^{p} \text { for } a \in[r]\right\} \subseteq \operatorname{Hom}\left(\mathcal{V}_{p}, \mathcal{Q}_{p}\right)
$$


It is easy to see that codimension of the vector subspace $B_{p} \subseteq \operatorname{Hom}\left(\mathcal{V}_{p}, \mathcal{Q}_{p}\right)$ is $\operatorname{codim}\left(\omega_{I^{p}}\right)$. Therefore

$$
\begin{aligned}
\operatorname{dim} & \operatorname{Hom}_{\mathcal{I}}(\mathcal{V}, \mathcal{Q}, \mathcal{F}, \mathcal{G})=\operatorname{dim} \bigcap_{p \in \mathrm{S}} \pi_{p}^{-1}\left(B_{p}\right) \\
\geq & h^{0}\left(\mathbb{P}^{1}, \mathcal{H o m}(\mathcal{V}, \mathcal{Q})\right)-\sum_{p \in \mathrm{S}} \operatorname{codim}\left(\omega_{I^{p}}\right) \\
& =\chi(d, r, D-d, n-r)+h^{1}\left(\mathbb{P}^{1}, \mathcal{H o m}(\mathcal{V}, \mathcal{Q})\right)-\sum_{p \in \mathrm{S}} \operatorname{codim}\left(\omega_{I^{p}}\right) \\
& =\operatorname{dim} \mathcal{I}+h^{1}\left(\mathbb{P}^{1}, \mathcal{H o m}(\mathcal{V}, \mathcal{Q})\right) .
\end{aligned}
$$

This proves (1). It is easy to deduce (2) from (1) and linear algebra. The assertion in (3) is a trivial consequence of (1) and (2).

Proposition 5.5. Let $\mathcal{I}=(d, r, D, n, I)$ be a Schubert state. Consider the following properties:

$(\alpha) \mathcal{I}$ is non-null.

$(\beta)$ For generic $(\mathcal{F}, \mathcal{G}) \in \mathrm{Fl}_{\mathrm{S}}\left(\mathcal{Z}_{d, r}\right) \times \mathrm{Fl}_{\mathrm{S}}\left(\mathcal{Z}_{D-d, n-r}\right)$, the dimension of the vector space $\operatorname{Hom}_{\mathcal{I}}\left(\mathcal{Z}_{d, r}, \mathcal{Z}_{D-d, n-r}, \mathcal{F}, \mathcal{G}\right)$ is $\operatorname{dim} \mathcal{I}$.

The following implications hold: $(\beta) \Rightarrow(\alpha)$ in any characteristic, and $(\alpha) \Rightarrow(\beta)$ in characteristic 0 .

Proof. $(\alpha) \Rightarrow(\beta)$ in characteristic 0 : Let $\mathcal{W}=\mathcal{Z}_{D, n}$. We claim that there exists $\mathcal{E} \in \mathrm{Fl}_{\mathrm{S}}(\mathcal{W})$ such that

(a) $\Omega^{o}(\mathcal{I}, \mathcal{W}, \mathcal{E})$ is a transverse non-empty intersection,

(b) $\Omega^{o}(\mathcal{I}, \mathcal{W}, \mathcal{E})$ has a dense subset of points $\mathcal{V}$ such that both $\mathcal{V}$ and $\mathcal{W} / \mathcal{V}$ are evenly split.

The first claim (a) is immediate from Kleiman's transversality theorem. To prove (b), let $U$ be the open subset of $\operatorname{Gr}(d, r, \mathcal{W})$ consisting of $\mathcal{V} \subseteq \mathcal{W}$ such that both $\mathcal{V}$ and $\mathcal{W} / \mathcal{V}$ are evenly split. This is non-empty by Proposition 2.2. By Kleiman's transversality theorem each irreducible component of the intersection of $\Omega^{\circ}(\mathcal{I}, \mathcal{W}, \mathcal{E})$ with any irreducible component of the complement of $U$ is of the expected dimension, which is less than $\operatorname{dim} \mathcal{I}$. Therefore $\Omega^{\circ}(\mathcal{I}, \mathcal{W}, \mathcal{E})$ has a dense intersection with $U$, which yields (b).

Now pick a $\mathcal{V}$ as in $(b)$. Choose isomorphisms $\mathcal{V} \stackrel{\sim}{\rightarrow} \mathcal{Z}_{d, r}$ and $\mathcal{W} / \mathcal{V} \stackrel{\sim}{\rightarrow} \mathcal{Z}_{D-d, n-r}$. By property (a) above, $T\left(\Omega^{\circ}(\mathcal{I}, \mathcal{W}, \mathcal{E})\right)_{\mathcal{V}}$ is of dimension $\operatorname{dim} \mathcal{I}$. Hence, using Lemma 5.2 and Lemma $5.4(3)$, we see that $(\beta)$ holds.

$(\beta) \Rightarrow(\alpha)$ in any characteristic: Let $(\mathcal{F}, \mathcal{G})$ be as in $(\beta)$. Using Lemma $5.4(3)$, $H^{1}\left(\mathbb{P}^{1}, \operatorname{Hom}\left(\mathcal{Z}_{d, r}, \mathcal{Z}_{D-d, n-r}\right)\right)=0$ and therefore by Corollary $12.3, \operatorname{Gr}\left(d, r, \mathcal{Z}_{D, n}\right)$ $\neq \emptyset$. Using Proposition 2.2, there exists an evenly split subbundle $\mathcal{V} \subseteq \mathcal{Z}_{D, n}$ such that the quotient $\mathcal{Q}=\mathcal{Z}_{D, n} / \mathcal{V}$ is also evenly split. Choose isomorphisms $\mathcal{V} \stackrel{\sim}{\rightarrow} \mathcal{Z}_{d, r}$ and $\mathcal{W} / \mathcal{V} \stackrel{\sim}{\rightarrow} \mathcal{Z}_{D-d, n-r}$ and use these to identify $(\mathcal{V}, \mathcal{Q})$ with $\left(\mathcal{Z}_{d, r}, \mathcal{Z}_{D-d, n-r}\right)$.

By an easy computation at each $p \in \mathrm{S}$ (see e.g. [6], Lemma 2.5), it is easy to see that there exists $\mathcal{E} \in \mathrm{Fl}_{\mathrm{S}}(\mathcal{W})$ such that

(1) $\mathcal{V} \in \Omega^{\circ}(\mathcal{I}, \mathcal{W}, \mathcal{E})$

(2) the flags induced by $\mathcal{E}$ on $\mathcal{V}$ and $\mathcal{Q}$ are $\mathcal{F}$ and $\mathcal{G}$, respectively.

Therefore by Lemma $5.2, \Omega^{\circ}(\mathcal{I}, \mathcal{W}, \mathcal{E})$ is a transverse and hence proper intersection at $\mathcal{V}$. By Lemma $5.6, \mathcal{I}$ is non-null. 
Lemma 5.6. Let $\mathcal{W}$ be an evenly split bundle, $\mathcal{E} \in \mathrm{Fl}_{\mathrm{S}}(\mathcal{W})$ and $\mathcal{V} \in \Omega^{\circ}(\mathcal{I}, \mathcal{W}, \mathcal{E})$. Suppose that each irreducible component of $\Omega^{\circ}(\mathcal{I}, \mathcal{W}, \mathcal{E})$ passing through $\mathcal{V}$ is of the expected dimension $(=\operatorname{dim} \mathcal{I})$. Then $\mathcal{I}$ is non-null.

Proof. The natural morphism $p: \mathcal{U}(\mathcal{I}, \mathcal{W}) \rightarrow \mathrm{Fl}_{\mathrm{S}}(\mathcal{W})$ is a morphism between smooth schemes (see Proposition 4.8). Using some standard results on flatness and fibres (see e.g. [24], Theorem 23.1), $p$ is flat at $\mathcal{V}$ and hence dominant. Therefore $\mathcal{I}$ is non-null.

\section{THE MAIN STRATEGY}

We will first outline the main ideas in the proof of $(B) \Rightarrow(A)$ in Theorem 2.8. We use a generalization of the strategy in [6]. Assume condition (B) in Theorem 2.8. Let $\mathcal{V}=\mathcal{Z}_{d, r}, \mathcal{Q}=\mathcal{Z}_{D-d, n-r}$ and let $(\mathcal{F}, \mathcal{G})$ be a generic point of $\operatorname{Fl}_{\mathrm{S}}(\mathcal{V}) \times \mathrm{Fl}_{\mathrm{S}}(\mathcal{Q})$.

Pick a generic element $\phi \in \operatorname{Hom}_{\mathcal{I}}(\mathcal{V}, \mathcal{Q}, \mathcal{F}, \mathcal{G})$. Set $\mathcal{S}=\operatorname{ker}(\phi)$. The image of $\phi$ is torsion free and hence locally free. Therefore $\mathcal{S}$ is a subbundle of $\mathcal{V}$. Let $\mathcal{K}=\left(d^{\prime}, r^{\prime}, d, r, K\right)$ be the (non-null) Schubert state such that $\mathcal{S} \in \Omega^{o}(\mathcal{K}, \mathcal{V}, \mathcal{F})$. In Section 9 we will prove that the dimension of $\operatorname{Hom}_{\mathcal{I}}(\mathcal{V}, \mathcal{Q}, \mathcal{F}, \mathcal{G})$ is given by the following formula:

$$
\operatorname{dim} \operatorname{Hom}_{\mathcal{I}}(\mathcal{V}, \mathcal{Q}, \mathcal{F}, \mathcal{G})=\operatorname{dim} \mathcal{I}+\operatorname{dim} \mathcal{K}+\left(\operatorname{dim} \mathcal{K}-\operatorname{dim} \mathcal{I}_{\mathcal{K}}\right) .
$$

By Remark 4.6 and assumption $(\mathrm{B}), \operatorname{dim} \mathcal{K}-\operatorname{dim} \mathcal{I}_{\mathcal{K}} \leq 0$. If $\operatorname{dim} \mathcal{K}=0$, then we conclude that $\operatorname{dim} \operatorname{Hom}_{\mathcal{I}}(\mathcal{V}, \mathcal{Q}, \mathcal{F}, \mathcal{G}) \leq \operatorname{dim} \mathcal{I}$. Using Proposition $5.5,(\beta) \Rightarrow(\alpha)$ and Lemma $5.4(3)$, we see that (A) holds in this case. We also find that in this case, $\operatorname{dim} \mathcal{I}_{\mathcal{K}}=0$.

If $\operatorname{dim} \mathcal{K}>0$, then $\Omega^{o}(\mathcal{K}, \mathcal{V}, \mathcal{F})$ is positive-dimensional at $\mathcal{S}$. Let $\eta: \mathcal{S} \rightarrow \mathcal{V} / \mathcal{S}$ be a generic element of the tangent space of $\Omega^{\circ}(\mathcal{K}, \mathcal{V}, \mathcal{F})$ at $\mathcal{S}$. Let $\mathcal{S}^{\prime}$ be the kernel of $\eta$, and suppose for simplicity that $\mathcal{S}^{\prime} \in \Omega^{\circ}(\mathcal{L}, \mathcal{S}, \mathcal{F}(\mathcal{S}))$ where $\operatorname{dim} \mathcal{L}=0$. (If $\operatorname{dim} \mathcal{L}>0$, we will have to pick a generic element in the tangent space of $\Omega^{o}(\mathcal{L}, \mathcal{S}, \mathcal{F}(\mathcal{S}))$ at $\mathcal{S}^{\prime}$ and iterate this process. $)$

Let $\mathcal{M}=\operatorname{im}(\phi) \subseteq \mathcal{Q}$ and $\mathcal{M}^{\prime}=\operatorname{im}(\phi \circ \eta) \subseteq \mathcal{Q}$. For simplicity assume that $\mathcal{M}$ and $\mathcal{M}^{\prime}$ are subbundles of $\mathcal{Q}$. Let $\mathcal{I}^{*}$ and $\mathcal{K}^{*}$ be Schubert states such that $\mathcal{M} \in \Omega^{o}\left(\mathcal{I}^{*}, \mathcal{Q}, \mathcal{G}\right)$ and $\mathcal{M}^{\prime} \in \Omega^{o}\left(\mathcal{K}^{*}, \mathcal{M}, \mathcal{G}(\mathcal{M})\right)$. Since $\mathcal{G} \in \mathrm{Fl}_{\mathrm{S}}(\mathcal{Q})$ is generic (see Lemma 6.2 ), $\operatorname{dim} \mathcal{K}^{*}-\operatorname{dim} \mathcal{I}_{\mathcal{K}^{*}}^{*} \leq 0$. This inequality, together with a suitable induction hypothesis and a small computation, yields (see Claim 8.1)

$$
\operatorname{dim} \operatorname{Hom}_{\mathcal{I}}(\mathcal{V}, \mathcal{Q}, \mathcal{F}, \mathcal{G}) \leq \operatorname{dim} \mathcal{I}+\left(\operatorname{dim} \mathcal{K}^{\prime}-\operatorname{dim} \mathcal{I}_{\mathcal{K}^{\prime}}\right)
$$

where $\mathcal{K}^{\prime}$ is the Schubert state such that $\mathcal{S}^{\prime} \in \Omega^{\circ}\left(\mathcal{K}^{\prime}, \mathcal{V}, \mathcal{F}\right)$. Now assumption (B) and Remark 4.6 imply that $\operatorname{dim} \operatorname{Hom}_{\mathcal{I}}(\mathcal{V}, \mathcal{Q}, \mathcal{F}, \mathcal{G}) \leq \operatorname{dim} \mathcal{I}$, as desired.

To make such an argument rigorous, we find it necessary to show that $\mathcal{S}$ and $\mathcal{V} / \mathcal{S}$ are evenly split vector bundles and that the induced pair of flags $(\mathcal{F}(\mathcal{S}), \mathcal{F}(\mathcal{V} / \mathcal{S})) \in$ $\mathrm{Fl}_{\mathrm{S}}(\mathcal{S}) \times \mathrm{Fl}_{\mathrm{S}}(\mathcal{V} / \mathcal{S})$ is suitably "generic". It could also very well happen that $\mathcal{M}$ and $\mathcal{M}^{\prime}$ are not subbundles of $\mathcal{Q}$. We will then replace $\mathcal{M}$ and $\mathcal{M}^{\prime}$ by their saturations in $\mathcal{Q}$ (see Section A.4). Frequently in the theory of vector bundles, the saturations of subsheaves have "better properties" than the subsheaves.

In Section 6.2, using some results from Section 6.1, we will write down a finite set of requirements on pairs of flags $(\mathcal{F}, \mathcal{G})$, such that the induced pair of flags $(\mathcal{F}(\mathcal{S}), \mathcal{F}(\mathcal{V} / \mathcal{S})) \in \mathrm{Fl}_{\mathrm{S}}(\mathcal{S}) \times \mathrm{Fl}_{\mathrm{S}}(\mathcal{V} / \mathcal{S})$ can also be assumed to have these properties. The reader may now move on to Section 7 on a first reading. 


\subsection{Bounds.}

Lemma 6.1. Let $D$ and $n>0$ be integers. Let $A$ be the set of pairs of (possibly null) Schubert states $(\mathcal{I}, \mathcal{K})$ of the form $\mathcal{I}=(d, r, D, n, I)$ and $\mathcal{K}=\left(d^{\prime}, r^{\prime}, d, r, K\right)$, such that

(i) $\operatorname{Gr}\left(d, r, \mathcal{Z}_{D, n}\right) \neq \emptyset, \operatorname{Gr}\left(d^{\prime}, r^{\prime}, \mathcal{Z}_{D, n}\right) \neq \emptyset$,

(ii) inequality $\left(\dagger_{\mathcal{K}}^{\mathcal{I}}\right)$ fails to hold.

Then the set $A$ is finite.

Proof. Consider the cases:

- $n-r>0$ and $r^{\prime}>0$ : It is easy to see that the degrees of coherent subsheaves of a vector bundle on $\mathbb{P}^{1}$ are bounded above. Therefore, using hypothesis (i), we deduce that the integers $-d^{\prime}$ and $-d$ are bounded above. By inspecting the inequality $\left(\dagger^{\mathcal{I}}\right)$, we now see that there are only finitely many such $(\mathcal{I}, \mathcal{K}) \in A$.

- $n-r=0$ : This implies that $D=d$ and hence $\left(\dagger^{\mathcal{I}}\right)$ holds.

- $r^{\prime}=0$ : This implies that $d^{\prime}=0$ and hence $\left(\dagger_{\mathcal{K}}^{\mathcal{I}}\right)$ holds.

Lemma 6.2. For every evenly split vector bundle $\mathcal{W}$ on $\mathbb{P}^{1}$, there exists a nonempty open subset $U \subseteq \mathrm{Fl}_{\mathrm{S}}(\mathcal{W})$ satisfying the following property: For every $\mathcal{E} \in U$ and subbundles $\mathcal{S} \subseteq \mathcal{V} \subseteq \mathcal{W}$, if we let $\mathcal{I}$ and $\mathcal{K}$ be the Schubert states such that $\mathcal{V} \in$ $\Omega^{o}(\mathcal{I}, \mathcal{W}, \mathcal{E})$ and $\mathcal{S} \in \Omega^{o}(\mathcal{K}, \mathcal{V}, \mathcal{E}(\mathcal{V}))$, then inequality $\left(\dagger_{\mathcal{K}}^{\mathcal{I}}\right)$ holds.

Proof. The proof uses the same ideas as the proof of $(\mathrm{A}) \Rightarrow(\mathrm{B})$ in Theorem 2.8. Let $A$ be the finite set of $(\mathcal{I}, \mathcal{K})$ from Lemma 6.1. Let $U$ be a non-empty open subset of $\mathrm{Fl}_{\mathrm{S}}(\mathcal{W})$ such that for $\mathcal{E} \in U, \Omega^{o}\left(\mathcal{I}_{\mathcal{K}}, \mathcal{W}, \mathcal{E}\right)$ is a (possibly empty) proper intersection, for every $(\mathcal{I}, \mathcal{K}) \in A$.

Let $\mathcal{E}, \mathcal{S}, \mathcal{V}, \mathcal{I}$ and $\mathcal{K}$ be as in the statement of the lemma and suppose inequality $\left(\uparrow_{\mathcal{K}}^{\mathcal{I}}\right)$ fails. From the inclusion $(4.1)$, we deduce that $\Omega^{o}\left(\mathcal{I}_{\mathcal{K}}, \mathcal{W}, \mathcal{E}\right) \neq \emptyset$ and hence $(\mathcal{I}, \mathcal{K}) \in A$. By Lemma 4.4 , the dimension of each irreducible component of $\Omega^{o}(\mathcal{K}, \mathcal{V}, \mathcal{E}(\mathcal{V}))$ is at least $\operatorname{dim} \mathcal{K}$. Since $(\mathcal{I}, \mathcal{K}) \in A, \Omega^{o}\left(\mathcal{I}_{\mathcal{K}}, \mathcal{W}, \mathcal{E}\right)$ is a proper intersection of dimension $\operatorname{dim} \mathcal{I}_{\mathcal{K}}$. By comparing the dimensions of the two sides of the inclusion (4.1), we conclude that inequality $\left(\dagger_{\mathcal{K}}^{\mathcal{I}}\right)$ holds (see Remark 4.6) and hence we reach a contradiction.

Remark 6.3. Notice that we are not requiring either $\mathcal{I}$ or $\mathcal{K}$ to be non-null in Lemma 6.2. Also, we allow the possibilities $\mathcal{V}=\mathcal{S}, \mathcal{W}=\mathcal{V}$ and $\mathcal{S}=0$.

Definition 6.4. For an evenly split vector bundle $\mathcal{W}$ on $\mathbb{P}^{1}$, define $B(\mathcal{W}) \subseteq \mathrm{Fl}_{\mathrm{S}}(\mathcal{W})$ to be the largest open subset $U \subseteq \mathrm{Fl}_{\mathrm{S}}(\mathcal{W})$ satisfying the conditions of Lemma 6.2.

\subsection{List of genericity properties.}

Lemma 6.5. The following objects (1) and (2), satisfying conditions (A) and (B) described below exist:

(1) for every pair $(\mathcal{V}, \mathcal{Q})$ of evenly split vector bundles of positive ranks on $\mathbb{P}^{1}$, a non-empty open subset $A(\mathcal{V}, \mathcal{Q}) \subseteq B(\mathcal{V}) \times B(\mathcal{Q}) \subseteq \mathrm{Fl}_{\mathrm{S}}(\mathcal{V}) \times \mathrm{Fl}_{\mathrm{S}}(\mathcal{Q})$ (see Section 6.1 for the definition of $B(\mathcal{V})$ and $B(\mathcal{Q})$ ),

(2) for every Schubert state of the form $\mathcal{I}=(d, r, D, n, I)$ where $0<r<n$, a non-null Schubert state of the form $\mathcal{K}(\mathcal{I})=\left(d^{\prime}(\mathcal{I}), r^{\prime}(\mathcal{I}), d, r, K(\mathcal{I})\right.$ ) (here $r^{\prime}(\mathcal{I})=r$ or $r^{\prime}(\mathcal{I})=0$ are possible). 
The conditions are: For every Schubert state $\mathcal{I}=(d, r, D, n, I)$, any point $(\mathcal{F}, \mathcal{G}) \in$ $A\left(\mathcal{Z}_{d, r}, \mathcal{Z}_{D-d, n-r}\right)$, setting $\mathcal{V}=\mathcal{Z}_{d, r}$ and $\mathcal{Q}=\mathcal{Z}_{D-d, n-r}$,

(A) for generic $\phi \in \operatorname{Hom}_{\mathcal{I}}(\mathcal{V}, \mathcal{Q}, \mathcal{F}, \mathcal{G})$, setting $\mathcal{S}=\operatorname{ker}(\phi)$,

(a) $\mathcal{S} \in \Omega^{\circ}(\mathcal{K}(\mathcal{I}), \mathcal{V}, \mathcal{F})$

(b) $\mathcal{S}$ and $\mathcal{V} / \mathcal{S}$ are evenly split vector bundles on $\mathbb{P}^{1}$,

(c) if $0<r^{\prime}(\mathcal{I})<r$, then $(\mathcal{F}(\mathcal{S}), \mathcal{F}(\mathcal{V} / \mathcal{S})) \in A(\mathcal{S}, \mathcal{V} / \mathcal{S})$,

(B) the dimension of $\operatorname{Hom}_{\mathcal{I}}(\mathcal{V}, \mathcal{Q}, \mathcal{F}, \mathcal{G})$ is given by the formula

$$
\operatorname{dim} \operatorname{Hom}_{\mathcal{I}}(\mathcal{V}, \mathcal{Q}, \mathcal{F}, \mathcal{G})=\operatorname{dim} \mathcal{I}+\operatorname{dim} \mathcal{K}(\mathcal{I})+\left(\operatorname{dim} \mathcal{K}(\mathcal{I})-\operatorname{dim} \mathcal{I}_{\mathcal{K}(\mathcal{I})}\right)
$$

The proof of Lemma 6.5 will be given in Section 9.3.

\section{The MAIN TECHNICAL RESUlt}

Theorem 7.1. Consider a 5-tuple of the form $(\mathcal{V}, \mathcal{Q}, \mathcal{I}, \mathcal{F}, \mathcal{G})$ where $\mathcal{V}$ is an evenly split vector bundle on $\mathbb{P}^{1}$ of degree $-d$ and rank $r, \mathcal{Q}$ an evenly split vector bundle on $\mathbb{P}^{1}$ of degree $d-D$ and rank $n-r,(\mathcal{F}, \mathcal{G})$ a generic point of $\mathrm{Fl}_{\mathrm{S}}(\mathcal{V}) \times \mathrm{Fl}_{\mathrm{S}}(\mathcal{Q})$ and $\mathcal{I}$ a Schubert state of the form $\mathcal{I}=(d, r, D, n, I)$.

We claim that there exists a filtration by vector subbundles

$$
\mathcal{S}^{(h)} \subsetneq \mathcal{S}^{(h-1)} \subsetneq \cdots \subsetneq \mathcal{S}^{(1)} \subsetneq \mathcal{S}^{(0)}=\mathcal{V}
$$

and morphisms $\eta_{u}: \mathcal{S}^{(u)} \rightarrow \mathcal{Q}$ with $\operatorname{ker}\left(\eta_{u}\right)=\mathcal{S}^{(u+1)}$ for $u=0, \ldots, h-1$, such that if we define Schubert states $\mathcal{K}(u)=\left(d_{u}, r_{u}, d, r, K(u)\right)$ for $u=1, \ldots, h$ by the requirement $\mathcal{S}^{(u)} \in \Omega^{o}(\mathcal{K}(u), \mathcal{V}, \mathcal{F})$, then

(i) $\mathcal{K}(1), \ldots, \mathcal{K}(h)$ are non-null Schubert states, and $\operatorname{dim} \mathcal{K}(h)=0$,

(ii) for $u=0, \ldots, h-1, p \in \mathrm{S}$ and $a \in[r]$,

$$
\left(\eta_{u}\right)_{p}\left(\mathcal{S}_{p}^{(u)} \cap F_{a}^{p}\right) \subseteq G_{i_{a}^{p}-a}^{p},
$$

(iii) the dimension of $\operatorname{Hom}_{\mathcal{I}}(\mathcal{V}, \mathcal{Q}, \mathcal{F}, \mathcal{G})$ is (see Remark 4.6 for an interpretation of the expression in parentheses)

$$
\operatorname{dim} \mathcal{I}+\left(\operatorname{dim} \mathcal{K}(h)-\operatorname{dim} \mathcal{I}_{\mathcal{K}(h)}\right) .
$$

Remark 7.2. (1) The theorem is valid for any $(\mathcal{F}, \mathcal{G}) \in A(\mathcal{V}, \mathcal{Q})$ (see Lemma 6.5).

(2) The following possibilities can occur: $h=0$ (in this case $\mathcal{S}^{(h)}=\mathcal{V}$ ) or $h>0$ and $\mathcal{S}^{(h)}=0$.

7.1. Proof of $(\mathrm{C}) \Rightarrow(\mathrm{A})$ in Theorem 2.8 assuming Theorem 7.1. Assume condition $(\mathrm{C})$. Let $\mathcal{V}=\mathcal{Z}_{d, r}, \mathcal{Q}=\mathcal{Z}_{D-d, n-r}$, and let $(\mathcal{F}, \mathcal{G})$ be a generic point of $\mathrm{Fl}_{\mathrm{S}}(\mathcal{V}) \times \mathrm{Fl}_{\mathrm{S}}(\mathcal{Q})$. Apply Theorem 7.1 to the 5 -tuple $(\mathcal{V}, \mathcal{Q}, \mathcal{I}, \mathcal{F}, \mathcal{G})$ and use the same notation. By conclusion (i) of Theorem 7.1, $\mathcal{K}(h)$ is a non-null Schubert state and since $\operatorname{dim} \mathcal{K}(h)=0,\langle\mathcal{K}(h)\rangle \neq 0$. Using conclusion (iii) of Theorem 7.1 and the hypothesis that $\left(\dagger_{\mathcal{K}(h)}^{\mathcal{I}}\right.$ ) holds (see Remark 2.10 for the cases $r_{h}=0$ or $r_{h}=r$ ), $\operatorname{dim} \operatorname{Hom}_{\mathcal{I}}(\mathcal{V}, \mathcal{Q}, \mathcal{F}, \mathcal{G}) \leq \operatorname{dim} \mathcal{I}$. We conclude the proof using Lemma $5.4(3)$ and Proposition $5.5(\beta) \Rightarrow(\alpha)$.

\section{Proof of Theorem 7.1}

The proof is by induction on $r$. If $\operatorname{Hom}_{\mathcal{I}}(\mathcal{V}, \mathcal{Q}, \mathcal{F}, \mathcal{G})=0$, the filtration is just the singleton $\mathcal{V}$ and $h=0$, so no morphisms $\eta$ needs to be given. Clearly, the condition in (iii) is satisfied.

Now assume that $\operatorname{Hom}_{\mathcal{I}}(\mathcal{V}, \mathcal{Q}, \mathcal{F}, \mathcal{G}) \neq 0$, and pick a generic element $\phi \in$ $\operatorname{Hom}_{\mathcal{I}}(\mathcal{V}, \mathcal{Q}, \mathcal{F}, \mathcal{G})$. Let $\mathcal{S}$ be the kernel of $\phi$, and let $\mathcal{K}=\left(d^{\prime}, r^{\prime}, d, r, K\right)$ be the 
non-null Schubert state $(\mathcal{K}$ is the same as $\mathcal{K}(\mathcal{I})$ from Lemma 6.5) defined by $\mathcal{S} \in \Omega^{o}(\mathcal{K}, \mathcal{V}, \mathcal{F})$ (therefore $\mathcal{S}$ is of degree $-d^{\prime}$ and rank $r^{\prime}$ ). Since $\mathcal{K}$ is non-null, $\operatorname{dim} \mathcal{K} \geq 0$.

If $\operatorname{dim} \mathcal{K}=0$ (which will be the case for example if $r=1$ ), take $\mathcal{S} \subsetneq \mathcal{V}$ (so that $h=1$ ) to be the filtration and $\eta_{0}=\phi: \mathcal{V} \rightarrow \mathcal{Q}$. Since $\operatorname{dim} \mathcal{K}=0$, (i) holds. From $\phi \in \operatorname{Hom}_{\mathcal{I}}(\mathcal{V}, \mathcal{Q}, \mathcal{F}, \mathcal{G})$, we deduce (ii). For (iii), according to equation (6.1), the dimension of $\operatorname{Hom}_{\mathcal{I}}(\mathcal{V}, \mathcal{Q}, \mathcal{F}, \mathcal{G})$ is $($ since $\operatorname{dim} \mathcal{K}=0$ )

$$
\operatorname{dim} \mathcal{I}+\left(\operatorname{dim} \mathcal{K}-\operatorname{dim} \mathcal{I}_{\mathcal{K}}\right) .
$$

Therefore assume $\operatorname{dim} \mathcal{K}>0$, which implies that $0<r^{\prime}<r$. Now by our discussion of genericity (see Lemma 6.5 and Remark 7.2) we may apply the induction hypothesis on the 5 -tuple $(\mathcal{S}, \mathcal{V} / \mathcal{S}, \mathcal{K}, \mathcal{F}(\mathcal{S}), \mathcal{F}(\mathcal{V} / \mathcal{S}))$.

We therefore find a filtration $\mathcal{S}^{(h)} \subsetneq \mathcal{S}^{(h-1)} \subsetneq \cdots \subsetneq \mathcal{S}^{(1)}=\mathcal{S}$ and morphisms $\gamma_{u}: \mathcal{S}^{(u)} \rightarrow \mathcal{V} / \mathcal{S}$ with $\operatorname{ker}\left(\gamma_{u}\right)=\mathcal{S}^{(u+1)}$ for $u=1, \ldots, h-1$ which satisfy the conclusions of Theorem 2.8 for the 5 -tuple $(\mathcal{S}, \mathcal{V} / \mathcal{S}, \mathcal{K}, \mathcal{F}(\mathcal{S}), \mathcal{F}(\mathcal{V} / \mathcal{S}))$.

We claim that the filtration

$$
\mathcal{S}^{(h)} \subsetneq \mathcal{S}^{(h-1)} \subsetneq \cdots \subsetneq \mathcal{S}^{(1)}=\mathcal{S} \subsetneq \mathcal{S}^{(0)}=\mathcal{V}
$$

and morphisms $\eta_{u}=\bar{\phi} \circ \gamma_{u}$ for $u=1, \ldots, h-1, \eta_{0}=\bar{\phi}$, satisfy conditions (i), (ii) and (iii) in the theorem (here $\bar{\phi}$ is the morphism $\mathcal{V} / \mathcal{S} \rightarrow \mathcal{Q}$ induced from $\phi$ ).

For $u=1, \ldots, h$, let $r_{u}$ be the rank of $\mathcal{S}^{(u)}$ and let $d_{u}=-\operatorname{deg}\left(\mathcal{S}^{(u)}\right)$ (so, $d^{\prime}=d_{1}$ and $\left.r^{\prime}=r_{1}\right)$. Let $\mathcal{L}(u)=\left(d_{u}, r_{u}, d^{\prime}, r^{\prime}, L(u)\right)$ be the Schubert state such that $\mathcal{S}^{(u)} \in \Omega^{o}(\mathcal{L}(u), \mathcal{S}, \mathcal{F}(\mathcal{S}))$. Now set $\mathcal{K}(u)=\mathcal{K}_{\mathcal{L}(u)}=\left(d_{u}, r_{u}, d, r, K(u)\right)$. By Remark 4.3, $\mathcal{S}^{(u)} \in \Omega^{o}(\mathcal{K}(u), \mathcal{V}, \mathcal{F})$.

Verification of (i): We know that $\mathcal{K}$ is non-null (Lemma 6.5) and by the induction hypothesis, each $\mathcal{L}(u)$ is non-null. Therefore by Corollary $4.10, \mathcal{K}(u)$ is non-null for $u=1, \ldots, h$.

According to inductive conclusion (iii) for the 5-tuple $(\mathcal{S}, \mathcal{V} / \mathcal{S}, \mathcal{K}, \mathcal{F}(\mathcal{S}), \mathcal{F}(\mathcal{V} / \mathcal{S})$,

$$
\operatorname{dim} \operatorname{Hom}_{\mathcal{K}}(\mathcal{S}, \mathcal{V} / \mathcal{S}, \mathcal{F}(\mathcal{S}), \mathcal{F}(\mathcal{V} / \mathcal{S}))=\operatorname{dim} \mathcal{K}+\left(\operatorname{dim} \mathcal{L}(h)-\operatorname{dim} \mathcal{K}_{\mathcal{L}(h)}\right)
$$

Since $\mathcal{F}$ is generic, $\operatorname{dim} \mathcal{L}(h) \leq \operatorname{dim} \mathcal{K}_{\mathcal{L}(h)}$ (use the assumption $\mathcal{F} \in B(\mathcal{V})$, Lemma 6.2 and Remark 4.6). But, by Lemma 5.4,

$$
\operatorname{dim} \operatorname{Hom}_{\mathcal{K}}(\mathcal{S}, \mathcal{V} / \mathcal{S}, \mathcal{F}(\mathcal{S}), \mathcal{F}(\mathcal{V} / \mathcal{S})) \geq \operatorname{dim} \mathcal{K}
$$

We therefore conclude that $\operatorname{dim} \mathcal{L}(h)=\operatorname{dim} \mathcal{K}_{\mathcal{L}(h)}$. Inductive conclusion (i) for $\mathcal{K}$ says that $\operatorname{dim} \mathcal{L}(h)=0$. Therefore $\operatorname{dim} \mathcal{K}(h)=\operatorname{dim} \mathcal{K}_{\mathcal{L}(h)}=0$ as desired.

Verification of (ii): We need to verify that for $u=0, \ldots, h-1, p \in \mathrm{S}$ and $a \in[r]$,

$$
\left(\eta_{u}\right)_{p}\left(\mathcal{S}_{p}^{(u)} \cap F_{a}^{p}\right) \subseteq G_{i_{a}^{p}-a}^{p}
$$

Now suppose $u, p$ and $a$ are as above. If $u=0$, inclusion (8.1) is clear because $\phi \in \operatorname{Hom}_{\mathcal{I}}(\mathcal{V}, \mathcal{Q}, \mathcal{F}, \mathcal{G})$. So assume $u>0$ and let $t$ be such that $F_{a}^{p} \cap \mathcal{S}_{p}=F_{t}^{p}(\mathcal{S})$. Clearly, $k_{t}^{p} \leq a$.

From $\eta_{u}=\bar{\phi} \circ \gamma_{u}$, we see that

$$
\left(\eta_{u}\right)_{p}\left(\mathcal{S}_{p}^{(u)} \cap F_{a}^{p}\right)=\bar{\phi}_{p}\left(\gamma_{u}\right)_{p}\left(\mathcal{S}_{p}^{(u)} \cap F_{t}^{p}(\mathcal{S})\right) \subseteq \bar{\phi}_{p}\left(F_{k_{t}^{p}-t}^{p}(\mathcal{V} / \mathcal{S})\right)
$$

where in the last inclusion we have used property (ii) satisfied by the morphisms $\gamma_{u}:\left(\gamma_{u}\right)_{p}\left(\mathcal{S}_{p}^{(u)} \cap F_{t}^{p}(\mathcal{S})\right) \subseteq F_{k_{t}^{p}-t}^{p}(\mathcal{V} / \mathcal{S})$. But,

$$
\bar{\phi}_{p}\left(F_{k_{t}^{p}-t}^{p}(\mathcal{V} / \mathcal{S})\right)=\bar{\phi}_{p}\left(\left(F_{k_{t}^{p}}^{p}+\mathcal{S}_{p}\right) / \mathcal{S}_{p}\right)=\phi_{p}\left(F_{k_{t}^{p}}^{p}\right) \subseteq \phi_{p}\left(F_{a}^{p}\right) \subseteq G_{i_{a}^{p}-a}^{p} .
$$


Verification of (iii): We claim,

Claim 8.1. For $u=1, \ldots, h$, let

$$
b(u)=\operatorname{dim} \mathcal{K}(u)-\operatorname{dim} \mathcal{L}(u)+\left(\operatorname{dim} \mathcal{K}(u)-\operatorname{dim} \mathcal{I}_{\mathcal{K}(u)}\right) .
$$

Then $b(u) \leq b(u+1)$ for $u=1, \ldots, h-1$.

The claim implies that $b(h) \geq b(1)$. But $\operatorname{dim} \mathcal{L}(1)=0$ and therefore by equation $(6.1), \operatorname{dim} \operatorname{Hom}_{\mathcal{I}}(\mathcal{V}, \mathcal{Q}, \mathcal{F}, \mathcal{G})=b(1)+\operatorname{dim} \mathcal{I}$. Hence, $\operatorname{dim} \operatorname{Hom}_{\mathcal{I}}(\mathcal{V}, \mathcal{Q}, \mathcal{F}, \mathcal{G}) \leq$ $\operatorname{dim} \mathcal{I}+b(h)$. Also, from (i) and induction, $\operatorname{dim} \mathcal{K}(h)=\operatorname{dim} \mathcal{L}(h)=0$. Therefore the dimension of $\operatorname{Hom}_{\mathcal{I}}(\mathcal{V}, \mathcal{Q}, \mathcal{F}, \mathcal{G})$ is less than or equal to the integer (7.1).

But according to Lemma 8.6, the dimension of $\operatorname{Hom}_{\mathcal{I}}(\mathcal{V}, \mathcal{Q}, \mathcal{F}, \mathcal{G})$ is at least as much as the integer (7.1). In conjunction with the above, this says that the dimension of $\operatorname{Hom}_{\mathcal{I}}(\mathcal{V}, \mathcal{Q}, \mathcal{F}, \mathcal{G})$ is equal to the integer (7.1). The proof of Theorem 7.1 would therefore be complete once the claim is proved.

Proof of the claim: For $u=0, \ldots, h$, let $\mathcal{M}^{(u)}$ be the saturation of $\operatorname{im}\left(\eta_{u}\right)$ in $\mathcal{Q}$, and let $\mathcal{M}=\mathcal{M}^{(0)}$ be the saturation of $\operatorname{im}(\phi)$ in $\mathcal{Q}$ (see Section A.4 for the concept of the saturation of a coherent subsheaf of a vector bundle).

The inequality $b(u) \leq b(u+1)$ in Claim 8.1 will be obtained by applying the defining property of $\mathcal{G} \in B(\mathcal{Q})$ to $\mathcal{M}^{(u)} \subseteq \mathcal{M}^{(0)}=\mathcal{M} \subseteq \mathcal{Q}$ (see Definition 6.4). If $\operatorname{im}\left(\eta_{u}\right)$ and $\operatorname{im}(\phi)$ were subbundles of $\mathcal{Q}$, then the proof of Claim 5.2 in [6] carries over immediately. We will carefully keep track, using the concept of parabolic bundles, of how far $\mathcal{M}$ and $\mathcal{M}^{\prime}$ are from being subbundles and show that the contributions of the "discrepancies" are such that we can still arrive at the desired conclusion. We will use some homological algebra-type techniques from the theory of parabolic bundles in dealing with the inequalities $\left(\dagger_{\mathcal{K}}^{\mathcal{I}}\right)$ (see e.g. Lemma A.4).

8.1. Parabolic bundles and the Horn problem. Let $\mathcal{W}=\mathcal{Z}_{D, n}$, and let $\mathcal{V} \subsetneq \mathcal{W}$ be a subbundle. Pick $\mathcal{E} \in \mathrm{Fl}_{\mathrm{S}}(\mathcal{W})$ and let $\mathcal{I}=(d, r, D, n, I)$ be the Schubert state such that $\mathcal{V} \in \Omega^{o}(\mathcal{I}, \mathcal{W}, \mathcal{E})$. Consider the parabolic bundle $\mathcal{V}^{\dagger}=(\mathcal{V}, \mathcal{E}(\mathcal{V}), w)$ (see Section A.5 for the notation and basic results) where $w_{a}^{p}=\frac{n-r+a-i_{a}^{p}}{n-r}$ for $p \in \mathrm{S}$ and $a \in[r]$. Let $\mathcal{S} \subseteq \mathcal{V}$ be a subbundle and let $\mathcal{K}=\left(d^{\prime}, r^{\prime}, d, r, K\right)$ be the Schubert state such that $\mathcal{S} \in \Omega^{\circ}(\mathcal{K}, \mathcal{V}, \mathcal{E}(\mathcal{V}))$. Then,

$$
\begin{aligned}
(n-r) \operatorname{pardeg}\left(\mathcal{S}, \mathcal{V}^{\dagger}\right)= & -(n-r) d^{\prime}+\sum_{p \in \mathrm{S}} \sum_{a \in K^{p}}\left(n-r+a-i_{a}^{p}\right) \\
= & \left(-(n-r) d^{\prime}+\chi\left(d^{\prime}, r^{\prime}, D-d, n-r\right)\right) \\
& +\left(-\chi\left(d^{\prime}, r^{\prime}, D-d, n-r\right)+\sum_{p \in \mathrm{S}} \sum_{a \in K^{p}}\left(n-r+a-i_{a}^{p}\right)\right) \\
= & \chi\left(0, r^{\prime}, D-d, n-r\right)+\left(\operatorname{dim} \mathcal{K}-\operatorname{dim} \mathcal{I}_{\mathcal{K}}\right) .
\end{aligned}
$$

We conclude that if $\mathcal{W}$ is evenly split and $\mathcal{E} \in B(\mathcal{W})$ (see Definition 6.4 and Remark 4.6), then

$$
(n-r) \operatorname{pardeg}\left(\mathcal{S}, \mathcal{V}^{\dagger}\right) \leq \chi\left(0, r^{\prime}, D-d, n-r\right) .
$$

8.2. Return to the proof of Claim 8.1. Let $\mathcal{M}^{\dagger}$ be the induced parabolic bundle corresponding to the subbundle $\mathcal{M} \subseteq \mathcal{Q}$ and let $\mathcal{G} \in \mathrm{Fl}_{\mathrm{S}}(\mathcal{Q})$ (see Section 8.1).

For $p \in \mathrm{S}$, let $\epsilon(p)$ be the dimension of the kernel of $\operatorname{im}(\phi)_{p} \rightarrow \mathcal{Q}_{p}$. Using the isomorphism $\mathcal{V} / \mathcal{S} \stackrel{\sim}{\rightarrow} \operatorname{im}(\phi)$ and the snake lemma, we see that the dimension of 
$\operatorname{ker}\left(\phi_{p}\right) \supseteq \mathcal{S}_{p}$ is $r^{\prime}+\epsilon(p)$ (recall that the rank of $\mathcal{S}$ is $r^{\prime}$ ). Let $H^{p}=\left\{h_{1}^{p}<\cdots<\right.$ $\left.h_{r^{\prime}+\epsilon(p)}^{p}\right\} \in\left(\begin{array}{c}{[r]} \\ r^{\prime}+\epsilon(p)\end{array}\right)$ be such that

$$
\operatorname{ker}\left(\phi_{p}\right) \in \Omega_{H^{p}}^{o}\left(F_{\bullet}^{p}\right) \subseteq \operatorname{Gr}\left(r^{\prime}+\epsilon(p), \mathcal{V}_{p}\right) .
$$

Introduce the notation

$$
\begin{gathered}
\theta^{p}(a)=i_{a}^{p}-a \text { for } p \in \mathrm{S}, a \in[r], \\
D^{*}=D-d, \quad n^{*}=n-r, \quad r^{*}=r-r^{\prime}, \quad d^{*}=d-d^{\prime}, \\
d_{\mathcal{M}}=-\operatorname{deg}(\mathcal{M}), \quad c=\frac{1}{n-r-\left(r-r^{\prime}\right)}=\frac{1}{n^{*}-r^{*}} .
\end{gathered}
$$

Remark 8.2. We think of $(\mathcal{W}, \mathcal{V})$ from Section 4 as being replaced by $(\mathcal{Q}, \mathcal{M})$ for the calculation. Therefore we have denoted the degree and rank of $\mathcal{Q}$ by $-D^{*}$ and $n^{*}$, respectively, and the rank of $\mathcal{M}$ as $r^{*}$. The degree of $\mathcal{M}$ is (at least) $-d^{*}$ plus some contributions coming from $\epsilon(p)$ (Lemma 8.3).

The fundamental inequality that we are going to use is equation (8.4) with $(\mathcal{S}, \mathcal{V}, \mathcal{W}, \mathcal{E})$ replaced by $\left(\mathcal{M}^{(u)}, \mathcal{M}, \mathcal{Q}, \mathcal{G}\right)$ (since $\mathcal{G} \in B(\mathcal{Q})$ by assumption):

$$
\left(n^{*}-r^{*}\right) \operatorname{pardeg}\left(\mathcal{M}^{(u)}, \mathcal{M}^{\dagger}\right)-\chi\left(0, r_{u}-r_{u+1}, D^{*}-d_{\mathcal{M}}, n^{*}-r^{*}\right) \leq 0 .
$$

(Recall that $\mathcal{M}$ is a vector bundle of degree $-d_{\mathcal{M}}$ and rank $r^{*}$ on $\mathbb{P}^{1}$.)

Before we use inequality (8.6), we note some crucial inequalities.

Lemma 8.3. $-d_{\mathcal{M}} \geq-d^{*}+\sum_{p \in \mathrm{S}} \epsilon(p)$.

Proof. Let $\mathcal{C}$ be the cokernel of the injective morphism of sheaves $\mathcal{V} / \mathcal{S} \rightarrow \mathcal{M}$. It is easy to see that $\mathcal{C}$ is a torsion sheaf such that $\mathcal{C} \otimes k(p)$ has dimension $\epsilon(p)$ for $p \in S$. Therefore $h^{0}(\mathcal{C}) \geq \sum_{p \in \mathrm{S}} \epsilon(p)$. The inequality now follows from the equality of Euler characteristics $\chi(\mathcal{M})=\chi(\mathcal{V} / \mathcal{S})+\chi(\mathcal{C})$ and the Riemann-Roch theorem $\left(\right.$ note that $\left.h^{1}(\mathcal{C})=0\right)$.

Lemma 8.4. For $a \in\left[r^{\prime}+\epsilon(p)\right]$ and $p \in \mathrm{S}$, the weight attached to $\mathcal{M}_{p} \cap G_{\theta^{p}\left(h_{a}^{p}\right)}^{p}\left(\mathcal{Q}_{p}\right)$ (which is a member of the induced flag $G_{\bullet}^{p}(\mathcal{M})$ ) in the parabolic bundle $\mathcal{M}^{\dagger}$ is at least

$$
c\left(n^{*}-r^{*}+\left(h_{a}^{p}-a\right)-\theta^{p}\left(h_{a}^{p}\right)\right) .
$$

Proof. Fix $p \in \mathrm{S}$ and suppose $\mathcal{M}_{p} \in \Omega_{J}^{o}\left(G_{\bullet}^{p}\right)$ where $J=\left\{j_{1}<\cdots<j_{r^{*}}\right\} \in\left(\begin{array}{c}{\left[n^{*}\right]} \\ r^{*}\end{array}\right)$. Now, if $\operatorname{dim}\left(\mathcal{M}_{p} \cap G_{\theta^{p}\left(h_{a}^{p}\right)}^{p}\left(\mathcal{Q}_{p}\right)\right)=x$ and $x \neq 0$, then $j_{x} \leq \theta^{p}\left(h_{a}^{p}\right)$. Also, $x \geq h_{a}^{p}-a$ because

(1) $\operatorname{dim} \phi_{p}\left(F_{h_{a}^{p}}^{p}\left(\mathcal{V}_{p}\right)\right)=h_{a}^{p}-a$,

(2) $\phi_{p}\left(F_{h_{a}^{p}}^{p}\left(\mathcal{V}_{p}\right)\right) \subseteq \mathcal{M}_{p} \cap G_{\theta^{p}\left(h_{a}^{p}\right)}^{p}\left(\mathcal{Q}_{p}\right)$.

The weight attached to $\mathcal{M}_{p} \cap G_{\theta^{p}\left(h_{a}^{p}\right)}^{p}\left(\mathcal{Q}_{p}\right)$ is $c\left(n^{*}-r^{*}+x-j_{x}\right)$ which is at least as much as the rational number (8.7). If $x=0$, which could happen only when $h_{a}^{p}=a$,

$$
c\left(n^{*}-r^{*}+\left(h_{a}^{p}-a\right)-\theta^{p}\left(h_{a}^{p}\right)\right) \leq c\left(n^{*}-r^{*}\right)=1
$$

and the weight attached is $\geq 1$.

Lemma 8.5. The parabolic degree $\operatorname{pardeg}\left(\mathcal{M}^{(u)}, \mathcal{M}^{\dagger}\right)$ is at least

$$
\left(d_{u+1}-d_{u}\right)+c \sum_{p \in \mathrm{S}} \sum_{t \in L^{p}(u)-L^{p}(u+1)}\left(n^{*}-r^{*}+\left(k_{t}^{p}-t\right)-\theta^{p}\left(k_{t}^{p}\right)\right)-c \sum_{p \in \mathrm{S}} \epsilon(p)\left(r_{u}-r_{u+1}\right) .
$$


Proof. For $p \in \mathrm{S}$, suppose that

$$
\mathcal{S}_{p} \in \Omega_{U^{p}}^{o}\left(F_{\bullet}^{p}\left(\operatorname{ker}\left(\phi_{p}\right)\right)\right), U^{p}=\left\{u_{1}^{p}<\cdots<u_{r^{\prime}}^{p}\right\} \in\left(\begin{array}{c}
{\left[r^{\prime}+\epsilon(p)\right]} \\
r^{\prime}
\end{array}\right) .
$$

From the containment (8.5) it follows that $K^{p}=\left\{h_{u_{t}^{p}}^{p} \mid t=1, \ldots, r^{\prime}\right\}$ for $p \in \mathrm{S}$. Therefore $\phi_{p}$ maps $F_{k_{t}^{p}}^{p}\left(\mathcal{V}_{p}\right)$ to an element of the flag $G_{\bullet}^{p}\left(\mathcal{M}_{p}\right)$ whose weight is at least (by Lemma 8.4)

$$
\begin{aligned}
c\left(n^{*}-r^{*}+\left(h_{u_{t}^{p}}^{p}-u_{t}^{p}\right)-\theta^{p}\left(h_{u_{t}^{p}}^{p}\right)\right) & =c\left(n^{*}-r^{*}+k_{t}^{p}-u_{t}^{p}-\theta^{p}\left(k_{t}^{p}\right)\right) \\
& =c\left(n^{*}-r^{*}+k_{t}^{p}-t-\theta^{p}\left(k_{t}^{p}\right)\right)+c\left(t-u_{t}^{p}\right) \\
& \geq c\left(n^{*}-r^{*}+\left(k_{t}^{p}-t\right)-\theta^{p}\left(k_{t}^{p}\right)\right)-c \epsilon(p)
\end{aligned}
$$

where in the last step we have used the inequality $t-u_{t}^{p} \geq-\epsilon(p)$.

For $t=1, \ldots, r^{\prime}$, by the already verified (ii) in Theorem $7.1,\left(\eta_{u}\right)_{p}\left(F_{t}^{p}(\mathcal{S}) \cap\right.$ $\left.\mathcal{S}_{p}{ }^{(u)}\right) \subseteq \mathcal{M}_{p} \cap G_{\theta^{p}\left(k_{t}^{p}\right)}^{p}$. The statement now follows from Lemma A.4.

We will now return to the proof of the claim. Using Lemma 8.5 and inequality (8.6), we obtain the inequality (use $c\left(n^{*}-r^{*}\right)=1$ )

$$
\begin{aligned}
& \left(n^{*}-r^{*}\right)\left(d_{u+1}-d_{u}\right)+\sum_{p \in \mathrm{S}} \sum_{t \in L^{p}(u)-L^{p}(u+1)}\left(n^{*}-r^{*}+\left(k_{t}^{p}-t\right)-\theta^{p}\left(k_{t}^{p}\right)\right) \\
& -\sum_{p \in \mathrm{S}} \epsilon(p)\left(r_{u}-r_{u+1}\right)-\chi\left(0, r_{u}-r_{u+1}, D^{*}-d_{\mathcal{M}}, n^{*}-r^{*}\right) \leq 0 .
\end{aligned}
$$

The first, third and fourth terms in the above inequality combine to give

$$
\begin{aligned}
\left(n^{*}-r^{*}\right)\left(d_{u+1}-d_{u}\right)- & \sum_{p \in \mathrm{S}} \epsilon(p)\left(r_{u}-r_{u+1}\right)-\chi\left(0, r_{u}-r_{u+1}, D^{*}-d_{\mathcal{M}}, n^{*}-r^{*}\right) \\
& =-\chi\left(d_{u}-d_{u+1}, r_{u}-r_{u+1}, D^{*}-d_{\mathcal{M}}-\sum_{p \in \mathrm{S}} \epsilon(p), n^{*}-r^{*}\right) \\
& \geq-\chi\left(d_{u}-d_{u+1}, r_{u}-r_{u+1}, D^{*}-d^{*}, n^{*}-r^{*}\right)
\end{aligned}
$$

(using Lemma 8.3 in the last step, and note that $-\chi(d, r, b, m)$ is an increasing function of $b$ with other variables fixed).

We now deduce from inequalities (8.8) and (8.9) that

$$
\begin{aligned}
& \sum_{p \in \mathrm{S}} \sum_{t \in L^{p}(u)-L^{p}(u+1)}\left(n^{*}-r^{*}+\left(k_{t}^{p}-t\right)-\theta^{p}\left(k_{t}^{p}\right)\right) \\
&-\chi\left(d_{u}-d_{u+1}, r_{u}-r_{u+1}, D^{*}-d^{*}, n^{*}-r^{*}\right)
\end{aligned}
$$

$\leq 0$.

Write the left hand side of the inequality (8.10) as $A(u)-A(u+1)$ where

$$
\begin{aligned}
A(u) & =\sum_{p \in \mathrm{S}} \sum_{t \in L^{p}(u)}\left(n^{*}-r^{*}+\left(k_{t}^{p}-t\right)-\theta^{p}\left(k_{t}^{p}\right)\right)-\chi\left(d_{u}, r_{u}, D^{*}-d^{*}, n^{*}-r^{*}\right) \\
& =B(u)+C(u)
\end{aligned}
$$


with (see Remark 4.6)

$$
\begin{aligned}
B(u) & =\chi\left(d_{u}, r_{u}, d^{*}, r^{*}\right)-\sum_{p \in \mathrm{S}} \sum_{t \in L^{p}(u)}\left(r^{*}-\left(k_{t}^{p}-t\right)\right) \\
& =\operatorname{dim} \mathcal{K}(u)-\operatorname{dim} \mathcal{L}(u)
\end{aligned}
$$

and

$$
\begin{aligned}
C(u) & =-\chi\left(d_{u}, r_{u}, D^{*}, n^{*}\right)+\sum_{p \in \mathrm{S}} \sum_{t \in L^{p}(u)}\left(n^{*}-\theta^{p}\left(k_{t}^{p}\right)\right) \\
& =-\chi\left(d_{u}, r_{u}, D-d, n-r\right)+\sum_{p \in \mathrm{S}} \sum_{a \in K^{p}(u)}\left(n-r+a-i_{a}^{p}\right) .
\end{aligned}
$$

Therefore $b(u)=A(u)$, and we conclude the proof of Claim 8.1 by observing that inequality (8.10) gives $A(u) \leq A(u+1)$.

8.3. A dimension inequality. The following inequality was used in the proof of Theorem 7.1:

Lemma 8.6. Suppose that $\mathcal{V}, \mathcal{Q}, \mathcal{F}, \mathcal{G}$ and $\mathcal{I}$ are as in Definition 5.3. Let $\mathcal{K}$ be a Schubert state such that $\Omega^{\circ}(\mathcal{K}, \mathcal{V}, \mathcal{F}) \neq \emptyset$. Then,

$$
\operatorname{dim} \operatorname{Hom}_{\mathcal{I}}(\mathcal{V}, \mathcal{Q}, \mathcal{F}, \mathcal{G}) \geq \operatorname{dim} \mathcal{I}+\left(\operatorname{dim} \mathcal{K}-\operatorname{dim} \mathcal{I}_{\mathcal{K}}\right) .
$$

Proof. Let $\mathcal{K}=\left(d^{\prime}, r^{\prime}, d, r, K\right)$ and pick $\mathcal{S} \in \Omega^{o}(\mathcal{K}, \mathcal{V}, \mathcal{F})$. Then

$$
\operatorname{dim} \operatorname{Hom}(\mathcal{V} / \mathcal{S}, \mathcal{Q}) \geq \chi(\mathcal{H o m}(\mathcal{V} / \mathcal{S}, \mathcal{Q}))=\chi\left(d-d^{\prime}, r-r^{\prime}, D-d, n-r\right) .
$$

Let $T=\left\{\phi \in \operatorname{Hom}_{\mathcal{I}}(\mathcal{V}, \mathcal{Q}, \mathcal{F}, \mathcal{G}) \mid \phi(\mathcal{S})=0\right\}$. For each $p \in \mathrm{S}$ we have a morphism $\eta_{p}: \operatorname{Hom}(\mathcal{V} / \mathcal{S}, \mathcal{Q}) \rightarrow \operatorname{Hom}\left(\mathcal{V}_{p} / \mathcal{S}_{p}, \mathcal{Q}_{p}\right)$. Let

$$
W(p)=\left\{\psi \in \operatorname{Hom}\left(\mathcal{V}_{p} / \mathcal{S}_{p}, \mathcal{Q}_{p}\right) \mid \psi\left(F_{a}^{p}\right) \subseteq G_{i_{a}^{p}-a}^{p} \text { for } a \in[r]\right\} .
$$

The dimension of $W(p)$ is $\sum_{a \in[r] \backslash K^{p}}\left(i_{a}^{p}-a\right)$, and hence its codimension in $\operatorname{Hom}\left(\mathcal{V}_{p} / \mathcal{S}_{p}, \mathcal{Q}_{p}\right)$ is

$$
\left(r-r^{\prime}\right)(n-r)-\sum_{a \in[r] \backslash K^{p}}\left(i_{a}^{p}-a\right)=\sum_{a \in[r] \backslash K^{p}}\left(n-r+a-i_{a}^{p}\right) .
$$

Therefore

$$
\begin{aligned}
\operatorname{dim} T= & \operatorname{dim} \bigcap_{p \in \mathrm{S}} \eta_{p}^{-1}(W(p)) \\
\geq & \chi\left(d-d^{\prime}, r-r^{\prime}, D-d, n-r\right)-\sum_{p \in \mathrm{S}} \sum_{a \in[r] \backslash K^{p}}\left(n-r+a-i_{a}^{p}\right) \\
= & \chi(d, r, D-d, n-r)-\sum_{p \in \mathrm{S}} \sum_{a=1}^{r}\left(n-r+a-i_{a}^{p}\right) \\
& -\chi\left(d^{\prime}, r^{\prime}, D-d, n-r\right)+\sum_{p \in \mathrm{S}} \sum_{a \in K^{p}}\left(n-r+a-i_{a}^{p}\right),
\end{aligned}
$$

which gives the desired inequality 
8.4. Proof of Theorem 2.8. We have already proved $(\mathrm{A}) \Rightarrow(\mathrm{B})$ (Section 4) and $(\mathrm{C}) \Rightarrow(\mathrm{A})$ (Section 7.1 ). The implications $(\mathrm{B}) \Rightarrow(\mathrm{C}) \Rightarrow(\mathrm{D})$ are obvious and we are left with having to prove $(\mathrm{D}) \Rightarrow(\mathrm{B})$. We will assume the basic transversality result, Proposition 10.1 (which is independent of this section).

Let $\mathcal{I}=(d, r, D, n, I)$ be as in the statement of the theorem. Let $\mathcal{V}=\mathcal{Z}_{d, r}$ and pick a generic point $\mathcal{F} \in \mathrm{Fl}_{\mathrm{S}}(\mathcal{V})$. Consider the parabolic bundle $\mathcal{V}^{\dagger}=(\mathcal{V}, \mathcal{F}, w)$ where $w_{a}^{p}=\frac{n-r+a-i_{a}^{p}}{n-r}$ for $p \in \mathrm{S}$ and $a \in[r]$.

Let $\mathcal{S} \subseteq \mathcal{V}$ be a non-zero subbundle, and let $\mathcal{K}$ be the Schubert state such that $\mathcal{S} \in \Omega^{o}(\mathcal{K}, \mathcal{V}, \mathcal{F})$. Then by equation $(8.3)$,

$$
\operatorname{rk}(\mathcal{S})(n-r) \mu\left(\mathcal{S}, \mathcal{V}^{\dagger}\right)=\operatorname{rk}(\mathcal{S})((n-r)-(D-d))+\left(\operatorname{dim} \mathcal{K}-\operatorname{dim} \mathcal{I}_{\mathcal{K}}\right) .
$$

The assumption $\operatorname{dim} \mathcal{I} \geq 0$ implies that (take $\mathcal{K}$ to correspond to $\mathcal{S}=\mathcal{V}$ above)

$$
\mu\left(\mathcal{V}, \mathcal{V}^{\dagger}\right) \leq 1-\frac{D-d}{n-r}
$$

Now, suppose by way of contradiction that (D) holds and (B) fails, and let $\mathcal{K}$ be a non-null Schubert state for which inequality $\left(\dagger_{\mathcal{K}}^{\mathcal{I}}\right)$ fails. Let $\mathcal{S} \in \Omega^{o}(\mathcal{K}, \mathcal{V}, \mathcal{F})$. Then by equation (8.11),

$$
\mu\left(\mathcal{S}, \mathcal{V}^{\dagger}\right)>1-\frac{D-d}{n-r} \geq \mu\left(\mathcal{V}, \mathcal{V}^{\dagger}\right)
$$

Therefore $\mathcal{V}^{\dagger}$ is not a semistable parabolic bundle. Let $\mathcal{S}^{\prime} \subseteq \mathcal{V}$ be the HarderNarasimhan maximal contradictor of semistability (see [25]).

Let $\mathcal{K}^{\prime}$ be the Schubert state such that $\mathcal{S}^{\prime} \in \Omega^{o}\left(\mathcal{K}^{\prime}, \mathcal{V}, \mathcal{F}\right)$. By the uniqueness of $\mathcal{S}^{\prime}$, the genericity of $\mathcal{F}$ and Proposition 10.1 , we see that $\left\langle\mathcal{K}^{\prime}\right\rangle=1$. Therefore by assumption $(\mathrm{D})$, inequality $\left(\uparrow^{\mathcal{I}} \mathcal{K}^{\prime}\right)$ holds. Applying equation $(8.11)$ (with $\mathcal{S}$ replaced by $\mathcal{S}^{\prime}$ ), we obtain the following inequality which together with the inequality $\mu\left(\mathcal{S}^{\prime}, \mathcal{V}^{\dagger}\right) \geq \mu\left(\mathcal{S}, \mathcal{V}^{\dagger}\right)$ contradicts inequality $(8.12)$ :

$$
\operatorname{rk}\left(\mathcal{S}^{\prime}\right)(n-r) \mu\left(\mathcal{S}^{\prime}, \mathcal{V}^{\dagger}\right) \leq \operatorname{rk}\left(\mathcal{S}^{\prime}\right)((n-r)-(D-d)) .
$$

\section{Dimension counts And Genericity}

9.1. Dimension of $\operatorname{Hom}_{\mathcal{I}}(\mathcal{V}, \mathcal{Q}, \mathcal{F}, \mathcal{G})$. We will use notation from Definition 5.3. Let $\mathcal{V}$ and $\mathcal{Q}$ be evenly split vector bundles, and let $(\mathcal{F}, \mathcal{G})$ be a generic point of $\mathrm{Fl}_{\mathrm{S}}(\mathcal{V}) \times \mathrm{Fl}_{\mathrm{S}}(\mathcal{Q})$. Pick a generic element $\phi \in \operatorname{Hom}_{\mathcal{I}}(\mathcal{V}, \mathcal{Q}, \mathcal{F}, \mathcal{G})$ and let $\mathcal{S}=$ $\operatorname{ker}(\phi)$. We have already seen that $\mathcal{S}$ is a subbundle of $\mathcal{V}$ (see Section 6). Let $\mathcal{K}=\left(d^{\prime}, r^{\prime}, d, r, K\right)$ be the Schubert state such that $\mathcal{S} \in \Omega^{\circ}(\mathcal{K}, \mathcal{V}, \mathcal{F})$.

The aim of this section is to prove the following proposition:

Proposition 9.1. With notation and assumptions as above, $\operatorname{Hom}_{\mathcal{I}}(\mathcal{V}, \mathcal{Q}, \mathcal{F}, \mathcal{G})$ is of dimension

$$
\operatorname{dim} \mathcal{I}+\operatorname{dim} \mathcal{K}+\left(\operatorname{dim} \mathcal{K}-\operatorname{dim} \mathcal{I}_{\mathcal{K}}\right)
$$

Introduce for convenience, as in Section 8.2, the notation

$$
D^{*}=D-d, n^{*}=n-r, r^{*}=r-r^{\prime}, d^{*}=d-d^{\prime} .
$$

Let $\operatorname{Hom}\left(\mathcal{V}, \mathcal{Q}, d^{\prime}, r^{\prime}\right)$ be the space of morphisms $\mathcal{V} \rightarrow \mathcal{Q}$ such that the kernel is a subbundle of rank $r^{\prime}$ and degree $-d^{\prime}$ (the scheme structure will be given in Section 9.2). We postpone the proof of the following lemma to Section 9.2. 
Lemma 9.2. $\operatorname{Hom}\left(\mathcal{V}, \mathcal{Q}, d^{\prime}, r^{\prime}\right)$ is smooth and connected of dimension

$$
\operatorname{dim} \operatorname{Gr}\left(d^{\prime}, r^{\prime}, \mathcal{V}\right)+\chi\left(d^{*}, r^{*}, D^{*}, n^{*}\right) .
$$

For each $p \in \mathrm{S}$, let $Z(p)$ be the smooth scheme consisting of pairs $(C, \psi)$ where

- $C$ is an $r^{\prime}$-dimensional subspace of $\mathcal{V}_{p}$,

- $\psi$ is a morphism $\mathcal{V}_{p} / C \rightarrow \mathcal{Q}_{p}$ (not necessarily injective).

There is a morphism $\eta_{p}: \operatorname{Hom}\left(\mathcal{V}, \mathcal{Q}, d^{\prime}, r^{\prime}\right) \rightarrow Z(p)$ given by $\phi \mapsto\left((\operatorname{ker}(\phi))_{p}, \bar{\phi}_{p}\right)$ (where $\bar{\phi}: \mathcal{V} / \mathcal{S} \rightarrow \mathcal{Q}$ is the induced morphism). Let $T(p) \subseteq Z(p)$ be the subscheme of points $(C, \psi)$ such that

(1) for $a \in[r], \psi\left(F_{a}^{p}\right) \subseteq G_{i_{a}^{p}-a}^{p}$,

(2) $C \in \Omega_{K^{p}}^{o}\left(F_{\bullet}^{p}\right)$.

It is easy to see that $T(p) \rightarrow \Omega_{K^{p}}^{o}\left(F_{\bullet}^{p}\right)$ is a smooth morphism of fibre dimension $\sum_{a \in[r] \backslash K^{p}}\left(i_{a}^{p}-a\right)$. It now follows that $T(p)$ is smooth and

$$
\operatorname{codim}(T(p), Z(p))=\operatorname{codim}\left(\omega_{K^{p}}\right)+r^{*} n^{*}-\sum_{a \in[r] \backslash K^{p}}\left(i_{a}^{p}-a\right) .
$$

Let

$$
\Omega=\bigcap_{p \in \mathrm{S}} \eta_{p}^{-1}(T(p))
$$

By our hypothesis, $\Omega$ is a dense open subset of $\operatorname{Hom}_{\mathcal{I}}(\mathcal{V}, \mathcal{Q}, \mathcal{F}, \mathcal{G})$ and hence is irreducible. The dimension of $\Omega$ is at least

$$
\begin{aligned}
\operatorname{dim} \operatorname{Hom} & \left.\mathcal{V}, \mathcal{Q}, d^{\prime}, r^{\prime}\right)-\sum_{p \in \mathrm{S}} \operatorname{codim}(T(p), Z(p)) \\
= & \operatorname{dim} \operatorname{Gr}\left(d^{\prime}, r^{\prime}, \mathcal{Z}_{d, r}\right)+\chi\left(d^{*}, r^{*}, D^{*}, n^{*}\right) \\
& -\sum_{p \in \mathrm{S}}\left(\operatorname{codim}\left(\omega_{K^{p}}\right)+\left(r^{*} n^{*}-\sum_{a \in[r] \backslash K^{p}}\left(i_{a}^{p}-a\right)\right)\right) \\
= & \left(\operatorname{dim} \operatorname{Gr}\left(d^{\prime}, r^{\prime}, \mathcal{Z}_{d, r}\right)-\sum_{p \in \mathrm{S}} \operatorname{codim}\left(\omega_{K^{p}}\right)\right)+\left(\chi\left(d^{*}, r^{*}, D^{*}, n^{*}\right)\right. \\
& \left.\left.-\sum_{p \in \mathrm{S}} \sum_{a \in[r] \backslash K^{p}}\left(n^{*}+a-i_{a}^{p}\right)\right)\right) .
\end{aligned}
$$

We simplify this expression using the formulas for $\operatorname{dim} \mathcal{I}$ and $\operatorname{dim} \mathcal{K}$ and conclude that the dimension of $\Omega$ is at least

$$
\begin{aligned}
\operatorname{dim} \mathcal{K} & +\left(\chi\left(d, r, D^{*}, n^{*}\right)-\sum_{p \in \mathrm{S}} \sum_{a \in[r]}\left(n^{*}+a-i_{a}^{p}\right)\right. \\
& \left.-\chi\left(d^{\prime}, r^{\prime}, D^{*}, n^{*}\right)+\sum_{p \in \mathrm{S}} \sum_{a \in K^{p}}\left(n^{*}+a-i_{a}^{p}\right)\right) \\
= & \operatorname{dim} \mathcal{K}+\operatorname{dim} \mathcal{I}+\left(-\chi\left(d^{\prime}, r^{\prime}, D^{*}, n^{*}\right)+\sum_{p \in \mathrm{S}} \sum_{a \in K^{p}}\left(n^{*}+a-i_{a}^{p}\right)\right) .
\end{aligned}
$$

Therefore by Remark 4.6, the dimension of $\operatorname{Hom}_{\mathcal{I}}(\mathcal{V}, \mathcal{Q}, \mathcal{F}, \mathcal{G})$ is at least as much as the integer (9.1).

However, this only gives us an inequality. Kleiman's theorem cannot be applied because $Z(p)$ may not be homogenous for the group $\operatorname{GL}\left(\mathcal{V}_{p}\right) \times \operatorname{GL}\left(\mathcal{Q}_{p}\right)$.

But we can get an exact expression for the dimension of $\operatorname{Hom}_{\mathcal{I}}(\mathcal{V}, \mathcal{Q}, \mathcal{F}, \mathcal{G})$ if we keep track of the kernels of the morphisms $\bar{\phi}_{p}: \mathcal{V}_{p} / \mathcal{S}_{p} \rightarrow \mathcal{Q}_{p}$. For $p \in \mathrm{S}$, 
- as in Section 8.2, let $\epsilon(p)$ be the dimension of the kernel $\mathcal{B}(p) \subseteq \mathcal{V}_{p} / \mathcal{S}_{p}$ of the morphism $\bar{\phi}_{p}: \mathcal{V}_{p} / \mathcal{S}_{p} \rightarrow \mathcal{Q}_{p}$ induced from $\phi$,

- define $J^{p} \in\left(\begin{array}{c}{\left[r^{*}\right]} \\ \epsilon(p)\end{array}\right)$ from the requirement $\mathcal{B}(p) \in \Omega_{J^{p}}^{o}\left(F_{\bullet}^{p}(\mathcal{V} / \mathcal{S})\right)$.

Let $\operatorname{Hom}\left(\mathcal{V}, \mathcal{Q}, d^{\prime}, r^{\prime}, \epsilon\right)$ be the space of morphisms $\phi: \mathcal{V} \rightarrow \mathcal{Q}$ such that the kernel is a subbundle of rank $r^{\prime}$ and degree $-d^{\prime}$ and for $p \in \mathrm{S}$, the kernel of $\phi_{p}: \mathcal{V}_{p} \rightarrow \mathcal{Q}_{p}$ is of dimension $r^{\prime}+\epsilon(p)$. We postpone the proof of the following lemma to Section 9.2.

Lemma 9.3. $\operatorname{Hom}\left(\mathcal{V}, \mathcal{Q}, d^{\prime}, r^{\prime}, \epsilon\right)$ is smooth and connected of dimension

$$
\operatorname{dim} \operatorname{Gr}\left(d^{\prime}, r^{\prime}, \mathcal{V}\right)+\chi\left(d^{*}, r^{*}, D^{*}, n^{*}\right)-\sum_{p \in \mathrm{S}} \epsilon(p)\left(n^{*}-\left(r^{*}-\epsilon(p)\right)\right) .
$$

Let $\mathcal{A}=\operatorname{Hom}\left(\mathcal{V}, \mathcal{Q}, d^{\prime}, r^{\prime}, \epsilon\right)$. For each $p \in \mathrm{S}$, let $Y(p)$ be the smooth scheme consisting of triples $(C, B, \psi)$ where

- $C$ is an $r^{\prime}$-dimensional subspace of $\mathcal{V}_{p}$,

- $B$ is a $\epsilon(p)$-dimensional subspace of $\mathcal{V}_{p} / C$,

- $\psi$ is a morphism $\mathcal{V}_{p} / C \rightarrow \mathcal{Q}_{p}$ with kernel $B$.

There is a morphism $\lambda_{p}: \mathcal{A} \rightarrow Y(p)$ given by

$$
\lambda_{p}: \phi \mapsto\left((\operatorname{ker}(\phi))_{p}, \operatorname{ker}\left(\phi_{p}\right) /(\operatorname{ker}(\phi))_{p}, \bar{\phi}_{p}\right) .
$$

Clearly, the group $G L\left(\mathcal{V}_{p}\right) \times G L\left(\mathcal{Q}_{p}\right)$ acts transitively on $Y(p)$. For $p \in \mathrm{S}$, let $R(p) \subseteq Y(p)$ be the subscheme consisting of points $(C, B, \psi)$ such that

(1) $C \in \Omega_{K^{p}}^{o}\left(F_{\bullet}^{p}\right)$,

(2) $B \in \Omega_{J^{p}}^{o}\left(F_{\bullet}^{p}\left(\mathcal{V}_{p} / C\right)\right)$,

(3) for $a \in[r], \psi\left(F_{a}^{p}\right) \subseteq G_{i_{a}^{p}-a}^{p}$ (where we consider $\psi$ as a morphism $\mathcal{V}_{p} \rightarrow \mathcal{Q}_{p}$ ).

For $p \in \mathrm{S}$, let

$$
[r] \backslash K^{p}=\left\{\alpha^{p}(1)<\cdots<\alpha^{p}\left(r^{*}\right)\right\} .
$$

It is easy to see that the codimension of $R(p)$ in $Y(p)$ is (fibre $R(p)$ over the set of choices of $(C, B))$

$$
\operatorname{codim}\left(\omega_{K^{p}}\right)+\operatorname{codim}\left(\omega_{J^{p}}\right)+\left(\left(r^{*}-\epsilon(p)\right) n^{*}-\sum_{t \in\left[r^{*}\right] \backslash J^{p}}\left(i_{\alpha^{p}(t)}^{p}-\alpha^{p}(t)\right)\right) .
$$

Now,

$$
\sum_{t \in\left[r^{*}\right] \backslash J^{p}}\left(i_{\alpha^{p}(t)}^{p}-\alpha^{p}(t)\right)=\sum_{t \in\left[r^{*}\right]}\left(i_{\alpha^{p}(t)}^{p}-\alpha^{p}(t)\right)-\sum_{t \in J^{p}}\left(i_{\alpha^{p}(t)}^{p}-\alpha^{p}(t)\right)
$$

and

$$
\sum_{t \in\left[r^{*}\right]}\left(i_{\alpha^{p}(t)}^{p}-\alpha^{p}(t)\right)=\sum_{a \in[r] \backslash K^{p}}\left(i_{a}^{p}-a\right) .
$$

Therefore $\operatorname{codim}(R(p), Y(p))$ is

(9.6) $\operatorname{codim}\left(\omega_{K^{p}}\right)+\operatorname{codim}\left(\omega_{J^{p}}\right)-\epsilon(p) n^{*}+\sum_{a \in[r] \backslash K^{p}}\left(n^{*}+a-i_{a}^{p}\right)+\sum_{t \in J^{p}}\left(i_{\alpha^{p}(t)}^{p}-\alpha^{p}(t)\right)$.

It follows from our assumptions that $\bigcap_{p \in \mathrm{S}} \lambda_{p}^{-1}(R(p))$ for generic $\mathcal{F}$ and $\mathcal{G}$ contains a non-empty dense open subset of $\operatorname{Hom}_{\mathcal{I}}(\mathcal{V}, \mathcal{Q}, \mathcal{F}, \mathcal{G})$, of dimension (by Kleiman's theorem) $\operatorname{dim} \mathcal{A}-\sum_{p \in S} \operatorname{codim}(R(p), Y(p))$. Use the expression for $\operatorname{dim} \mathcal{A}$ given 
in Lemma 9.3 and equation (9.6) to write the dimension of the intersection $\bigcap_{p \in \mathrm{S}} \lambda_{p}^{-1}(R(p))$ as a sum of two parts. The first part is

$$
\operatorname{dim} \operatorname{Gr}\left(d^{\prime}, r^{\prime}, \mathcal{V}\right)-\sum_{p \in \mathrm{S}} \operatorname{codim}\left(\omega_{K^{p}}\right)+\chi\left(d^{*}, r^{*}, D^{*}, n^{*}\right)-\sum_{p \in \mathrm{S}} \sum_{a \in[r] \backslash K^{p}}\left(n^{*}+a-i_{a}^{p}\right)
$$

and the second part is the sum over $p \in \mathrm{S}$ of the "local discrepancies"

$$
\operatorname{Disc}(p)=-\epsilon(p)\left(n^{*}-\left(r^{*}-\epsilon(p)\right)\right)-\operatorname{codim}\left(\omega_{J^{p}}\right)+\epsilon(p) n^{*}-\sum_{t \in J^{p}}\left(i_{\alpha^{p}(t)}^{p}-\alpha^{p}(t)\right) .
$$

The first part is the same as the integer (9.1) (compare with equations (9.3) and (9.4)).

We will now show that $\operatorname{Disc}(p) \leq 0$ for $p \in S$. This will show that the dimension of $\operatorname{Hom}_{\mathcal{I}}(\mathcal{V}, \mathcal{Q}, \mathcal{F}, \mathcal{G})$ for generic $(\mathcal{F}, \mathcal{G})$ is less than or equal (hence equal) to the integer (9.1) and conclude the proof of Proposition 9.1.

Fix a $p \in \mathrm{S}$ and let $J^{p}=\left\{j_{1}<\cdots<j_{\epsilon(p)}\right\}$. Then

$$
\operatorname{codim}\left(\omega_{J^{p}}\right)=\sum_{\ell=1}^{\epsilon(p)}\left(r^{*}-\epsilon(p)+\ell-j_{\ell}\right) .
$$

Therefore

$$
\begin{aligned}
\operatorname{Disc}(p) & =\sum_{\ell=1}^{\epsilon(p)}\left(-n^{*}+\left(r^{*}-\epsilon(p)\right)-\left(r^{*}-\epsilon(p)+\ell-j_{\ell}\right)+n^{*}-\left(i_{\alpha^{p}\left(j_{\ell}\right)}^{p}-\alpha^{p}\left(j_{\ell}\right)\right)\right) \\
& =\sum_{\ell=1}^{\epsilon(p)}\left(j_{\ell}-\ell+\alpha^{p}\left(j_{\ell}\right)-i_{\alpha^{p}\left(j_{\ell}\right)}^{p}\right) .
\end{aligned}
$$

Pick $\phi \in \bigcap_{p \in \mathrm{S}} \lambda_{p}^{-1}(R(p)) \subseteq \operatorname{Hom}_{\mathcal{I}}(\mathcal{V}, \mathcal{Q}, \mathcal{F}, \mathcal{G})$. For each $t$, we have the inclusion

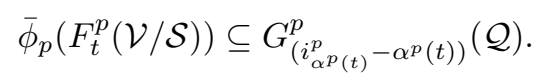

Take $t=j_{\ell}$. The dimension of the left hand side is $j_{\ell}-\ell$ and the right hand side is of dimension $i_{\alpha^{p}\left(j_{\ell}\right)}^{p}-\alpha^{p}\left(j_{\ell}\right)$. Therefore $j_{\ell}-\ell \leq i_{\alpha^{p}\left(j_{\ell}\right)}^{p}-\alpha^{p}\left(j_{\ell}\right)$ and hence $\operatorname{Disc}(p) \leq 0$.

9.2. Proofs of Lemma $\mathbf{9 . 2}$ and 9.3. Use notation from Section 9.1. We define $F\left(\mathcal{V}, \mathcal{Q}, d^{\prime}, r^{\prime}, \epsilon\right)$ to be the contravariant functor: (Schemes $\left./ \kappa\right) \rightarrow($ Sets) which assigns to a scheme $T$ over $\kappa$ the set of data of the form $(\mathcal{S}, \mathcal{B}, \phi)$ where

(a) $\mathcal{S}$ is a subbundle of $\mathcal{V}_{T}$ (the pull back of $\mathcal{V}$ to $\mathbb{P}^{1} \times T$ ) which is fibrewise, over $T$, of degree $-d^{\prime}$ and rank $r^{\prime}$,

(b) $\phi$ is a morphism $\mathcal{V}_{T} \stackrel{\phi}{\rightarrow} \mathcal{Q}_{T}$ with $\operatorname{ker}(\phi)=\mathcal{S}$, whose cokernel is flat over $T$,

(c) $\mathcal{B}=(\mathcal{B}(p) \mid p \in \mathrm{S})$ where for $p \in \mathrm{S}, \mathcal{B}(p)$ is a subbundle of $\left(\mathcal{V}_{T} / \mathcal{S}\right)_{p}=$ $\left.\left(\mathcal{V}_{T} / \mathcal{S}\right)\right|_{\{p\} \times T}$ of rank $\epsilon(p)$.

The above data is required to satisfy: For $p \in \mathrm{S}, \operatorname{ker}\left(\bar{\phi}_{p}\right)=\mathcal{B}(p)$ and $\bar{\phi}_{p}$ : $\left(\mathcal{V}_{T} / \mathcal{S}\right)_{p} \rightarrow\left(\mathcal{Q}_{T}\right)_{p}$ has a locally free cokernel (where $\bar{\phi}$ is the induced morphism $\left.\mathcal{V}_{T} / \mathcal{S} \rightarrow \mathcal{Q}_{T}\right)$.

Denote the functor obtained by considering pairs $(\mathcal{S}, \phi)$ satisfying (a) and (b) above by $F\left(\mathcal{V}, \mathcal{Q}, d^{\prime}, r^{\prime}\right)$. We make the following observation:

Lemma 9.4. Suppose that the pair $(\mathcal{S}, \phi)$ satisfies conditions (a) and (b). Then for $t \in T, H^{1}\left(\mathbb{P}^{1}, \mathcal{H o m}\left(\mathcal{V} / \mathcal{S}_{t}, \mathcal{Q}\right)\right)=0$. 
Proof. The morphism $\bar{\phi}_{t}: \mathcal{V} / \mathcal{S}_{t} \hookrightarrow \mathcal{Q}$ is a sheaf-theoretic injection of vector bundles. But $\mathcal{Q}$ is an evenly split vector bundle, so we can apply Lemma 9.7.

The functor (Schemes $/ \kappa) \rightarrow$ (Sets) which associates to a scheme $T$ over $\kappa$ the set $\operatorname{Hom}\left(\mathcal{V}_{T}, \mathcal{Q}_{T}\right)$ is represented by the $\kappa$-vector space $H=\operatorname{Hom}(\mathcal{V}, \mathcal{Q})$ (considered a scheme over $\kappa$ ). We have a universal morphism $\phi: \mathcal{V}_{H} \rightarrow \mathcal{Q}_{H}$ on $H$. Let $\mathcal{T}$ be the cokernel of this morphism. By the method of flattening stratifications ([26], Lecture 8) applied to $\mathcal{T}$ and each $\mathcal{T}_{p}$ for $p \in \mathrm{S}$, it is clear that each $F\left(\mathcal{V}, \mathcal{Q}, d^{\prime}, r^{\prime}, \epsilon\right)$ (resp. $\left.F\left(\mathcal{V}, \mathcal{Q}, d^{\prime}, r^{\prime}\right)\right)$ is representable by a scheme which we denote by $\operatorname{Hom}\left(\mathcal{V}, \mathcal{Q}, d^{\prime}, r^{\prime}, \epsilon\right)$ $\left(\right.$ resp. $\left.\operatorname{Hom}\left(\mathcal{V}, \mathcal{Q}, d^{\prime}, r^{\prime}\right)\right)$. These schemes coincide on the level of points with the sets of the same name in Section 9.1.

To motivate the functorial arguments in this section, let us first parameterize the points of $\operatorname{Hom}\left(\mathcal{V}, \mathcal{Q}, d^{\prime}, r^{\prime}\right)$ when both $\mathcal{V}$ and $\mathcal{Q}$ are evenly split vector bundles. That is, in the definition above, take $T=\operatorname{Spec} \kappa$. The set of choices of $\mathcal{S}$ satisfying condition (a) in the definition of the functor $F\left(\mathcal{V}, \mathcal{Q}, d^{\prime}, r^{\prime}\right)$ is parameterized by the smooth scheme $\operatorname{Gr}\left(d^{\prime}, r^{\prime}, \mathcal{V}\right)$.

Now we have to parameterize the possible choices $A(\mathcal{S})$ of $\phi: \mathcal{V} \rightarrow \mathcal{Q}$ satisfying condition (b). Consider the vector space $H(\mathcal{S})=\operatorname{Hom}(\mathcal{V} / \mathcal{S}, \mathcal{Q})$. We would not be interested in $\mathcal{S}$ if $H(\mathcal{S})$ did not contain a sheaf-theoretic injection. So suppose that $H(\mathcal{S})$ contains a sheaf-theoretic injection. By the vanishing of higher cohomology proven in Lemma 9.7, $H^{1}(\mathcal{H o m}(\mathcal{V} / \mathcal{S}, \mathcal{Q}))=0$ and hence the dimension of $H(\mathcal{S})$ equals $\chi(\mathcal{H o m}(\mathcal{V} / \mathcal{S}, \mathcal{Q}))$. Now, $A(\mathcal{S})$ is the open subset of $H(\mathcal{S})$ consisting of injections. The set of $\mathcal{S}$ such that $A(\mathcal{S})$ contains an injection is an open subset of $\operatorname{Gr}\left(d^{\prime}, r^{\prime}, \mathcal{V}\right)$.

To parameterize $\operatorname{Hom}\left(\mathcal{V}, \mathcal{Q}, d^{\prime}, r^{\prime}, \epsilon\right)$ when both $\mathcal{V}$ and $\mathcal{Q}$ are evenly split vector bundles, we proceed similarly: we select $\mathcal{S}$ and

$$
\mathcal{B}=(\mathcal{B}(p) \mid p \in \mathrm{S}) \in \prod_{p \in \mathrm{S}} \operatorname{Gr}\left(\epsilon(p), \mathcal{V}_{p} / \mathcal{S}_{p}\right)
$$

We then shift $\mathcal{V} / \mathcal{S}$ along $\mathcal{B}$ (see Section A.3) and consider morphisms $\Psi(\mathcal{V} / \mathcal{S}, \mathcal{B}) \rightarrow$ $\mathcal{Q}$. These morphisms are in one to one correspondence with morphisms $\phi: \mathcal{V} \rightarrow \mathcal{Q}$ with $\operatorname{ker}(\bar{\phi})=\mathcal{S}$ such that $\mathcal{B}(p) \subseteq \operatorname{ker}\left(\bar{\phi}_{p}\right)$ for each $p \in \mathrm{S}$. For (c), therefore, we want to consider the open subset of $\operatorname{Hom}(\Psi(\mathcal{V} / \mathcal{S}, \mathcal{B}), \mathcal{Q})$ consisting of injective morphisms such that the corresponding $\bar{\phi}_{p}: \mathcal{V}_{p} / \mathcal{S}_{p} \rightarrow \mathcal{Q}_{p}$ has kernel (exactly) $\mathcal{B}(p)$ for each $p \in \mathrm{S}$.

Lemmas 9.2 and 9.3 follow from

Lemma 9.5. Let $\mathcal{Y}$ be the universal quotient on $\mathbb{P}^{1} \times \operatorname{Gr}\left(d^{\prime}, r^{\prime}, \mathcal{V}\right)$.

(1) Consider the diagram

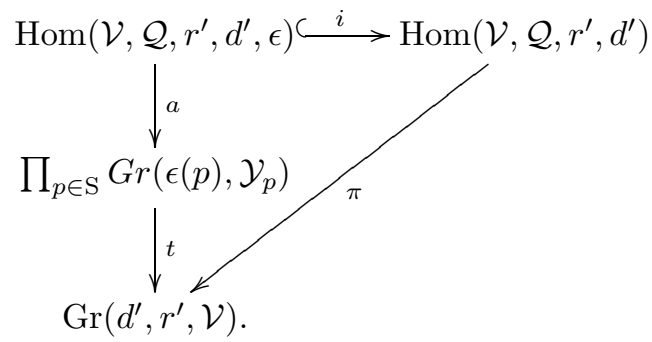


The morphisms $\pi$, $a$ and $t$ are each smooth with connected fibres and $i$ is an inclusion (here $\prod_{p \in \mathrm{S}} \operatorname{Gr}\left(\epsilon(p), \mathcal{Y}_{p}\right)$ is the fibre product, over $\operatorname{Gr}\left(d^{\prime}, r^{\prime}, \mathcal{V}\right)$, of the Grassmann bundles $\left.\operatorname{Gr}\left(\epsilon(p), \mathcal{Y}_{p}\right)\right)$.

(2) $\operatorname{Hom}\left(\mathcal{V}, \mathcal{Q}, r^{\prime}, d^{\prime}, \epsilon\right)$ is smooth and connected of dimension

$$
\operatorname{dim} \operatorname{Gr}\left(d^{\prime}, r^{\prime}, \mathcal{V}\right)+\chi\left(d^{*}, r^{*}, D^{*}, n^{*}\right)-\sum_{p \in \mathrm{S}} \epsilon(p)\left(n^{*}-\left(r^{*}-\epsilon(p)\right)\right) .
$$

(3) $\operatorname{Hom}\left(\mathcal{V}, \mathcal{Q}, r^{\prime}, d^{\prime}\right)$ is smooth and connected of dimension

$$
\operatorname{dim} \operatorname{Gr}\left(d^{\prime}, r^{\prime}, \mathcal{V}\right)+\chi\left(d^{*}, r^{*}, D^{*}, n^{*}\right) .
$$

Proof. Let $X=\operatorname{Gr}\left(d^{\prime}, r^{\prime}, \mathcal{V}\right)$. Consider the vector bundle $\mathcal{T}=\mathcal{H o m}\left(\mathcal{Y}, p_{\mathbb{P}^{1}}^{*} \mathcal{Q}\right)$ on $\mathbb{P}^{1} \times X$. Let $U_{X}$ be the open subset of $X$ consisting of points $x$ such that $H^{1}\left(\mathbb{P}^{1}, \mathcal{T}_{x}\right)=0$. Let $Y$ be the total space of $\left(p_{T}\right)_{*} \mathcal{T}$ over $U_{X}$. It is clear from the definition of $F\left(\mathcal{V}, \mathcal{Q}, d^{\prime}, r^{\prime}\right)$ and Lemmas A.1 and 9.4 that there is a natural morphism $\operatorname{Hom}\left(\mathcal{V}, \mathcal{Q}, d^{\prime}, r^{\prime}\right) \rightarrow Y$.

On $\mathbb{P}^{1} \times Y$ we have a morphism of sheaves

$$
\bar{\phi}: p_{\mathbb{P}^{1} \times X}^{*}(\mathcal{Y}) \rightarrow p_{\mathbb{P}^{1}}^{*} \mathcal{Q} .
$$

Let $U_{Y}$ be the open subset of points $y$ of $Y$ such that $\bar{\phi}_{y}$ is injective as a morphism of sheaves on $\mathbb{P}^{1} \times\{y\}$. By Lemmas A.1 and A.3, $U_{Y}$ represents $F\left(\mathcal{V}, \mathcal{Q}, d^{\prime}, r^{\prime}\right)$.

To represent $F\left(\mathcal{V}, \mathcal{Q}, d^{\prime}, r^{\prime}, \epsilon\right)$, form the Grassmann bundle

$$
\widetilde{X}=\prod_{p \in \mathrm{S}} \operatorname{Gr}\left(\epsilon(p), \mathcal{Y}_{p}\right)
$$

over $X$. Over $\tilde{X}$, for each $p \in \mathrm{S}$ we have vector bundles $\mathcal{B}(p) \subseteq p_{X}^{*}\left(\mathcal{Y}_{p}\right)$. We can therefore form the shift (a vector bundle on $\mathbb{P}^{1} \times \widetilde{X}$; see Section A.3) $\widetilde{\mathcal{Y}}=$ $\Psi\left(\mathcal{B}, p_{\mathbb{P}^{1} \times X}^{*} \mathcal{Y}\right)$. Let $\widetilde{\mathcal{T}}=\mathcal{H} \operatorname{om}\left(\widetilde{\mathcal{Y}}, p_{\mathbb{P}^{1}}^{*} \mathcal{Q}\right)$ on $\mathbb{P}^{1} \times \widetilde{X}$. Let $U_{\widetilde{X}}$ be the open subset of $\widetilde{X}$ consisting of points $\tilde{x}$ such that $H^{1}\left(\mathbb{P}^{1}, \widetilde{\mathcal{T}}_{\tilde{x}}\right)=0$. Let $\widetilde{Y}$ be the total space of $p_{\widetilde{X} *} \widetilde{\mathcal{T}}$ over $U_{\widetilde{X}}$. On $\mathbb{P}^{1} \times \widetilde{Y}$, there is a morphism

$$
\tilde{\phi}: p_{\mathbb{P}^{1} \times \widetilde{X}}^{*}(\widetilde{\mathcal{Y}}) \rightarrow p_{\mathbb{P}^{1}}^{*} \mathcal{Q} .
$$

Let $U_{\tilde{Y}}$ be the open subset of points $\tilde{y}$ of $\tilde{Y}$ such that $\tilde{\phi}_{\tilde{y}}$ is injective as a morphism of sheaves on $\mathbb{P}^{1} \times\{\tilde{y}\}$.

For each $p \in \mathrm{S}$, consider the (composite) morphism on $U_{\widetilde{Y}}$

$$
\bar{\phi}_{p}: p_{X}^{*}\left(\mathcal{Y}_{p}\right) \rightarrow p_{\widetilde{X}}^{*}\left(\widetilde{\mathcal{Y}}_{p}\right) \stackrel{\tilde{\phi}_{p}}{\rightarrow} \mathcal{Q}_{p} .
$$

This morphism has $p_{\widetilde{X}}^{*}(\mathcal{B}(p))$ in its kernel. We look at the open subset of $U_{\widetilde{Y}}$ consisting of points where the cokernel of $\bar{\phi}_{p}$ is locally free and the kernel is exactly $p_{\widetilde{X}}^{*}(\mathcal{B}(p))$ for each $p \in \mathrm{S}$. By Lemmas A.1 and A.3, this open subset represents $F\left(\mathcal{V}, \mathcal{Q}, d^{\prime}, r^{\prime}, \epsilon\right)$.

The smoothness assertions are clear from the above arguments. For the first dimension formula (the second one is obtained similarly), we need to add the (fibrewise over $\widetilde{X})$ Euler characteristic of $\mathcal{H o m}(\widetilde{\mathcal{Y}}, \mathcal{Q})$ to the dimension of the Grassmann bundle $\widetilde{X}$.

- The dimension of $\widetilde{X}$ is $\operatorname{dim} \operatorname{Gr}\left(d^{\prime}, r^{\prime}, \mathcal{V}\right)+\sum_{p \in \mathrm{S}} \epsilon(p)\left(r^{*}-\epsilon(p)\right)$. 
- The (fibrewise over $\widetilde{X}$ ) degree of $\widetilde{\mathcal{Y}}$ is $-d^{*}+\sum_{p \in S} \epsilon(p)$ and hence the fibrewise Euler characteristic of $\mathcal{H o m}(\widetilde{\mathcal{Y}}, \mathcal{Q})$ is $\chi\left(d^{*}, r^{*}, D^{*}, n^{*}\right)-\sum_{p \in \mathrm{S}} \epsilon(p) n^{*}$.

Remark 9.6. In Lemma 9.5, it is easy to see that $t$ is surjective. However $a$ and $\pi$ are smooth and dominant morphisms which need not be surjective. This is because the morphisms $U_{\widetilde{X}} \rightarrow \operatorname{Gr}\left(d^{\prime}, r^{\prime}, \mathcal{V}\right)$ and $U_{Y} \rightarrow \operatorname{Gr}\left(d^{\prime}, r^{\prime}, \mathcal{V}\right)$ may not be surjective.

Lemma 9.7. Let $\mathcal{V} \subseteq \mathcal{Z}_{D, n}$ be a coherent subsheaf. Then

(1) $\operatorname{Ext}^{1}\left(\mathcal{V}, \mathcal{Z}_{D, n}\right)=H^{1}\left(\mathbb{P}^{1}, \mathcal{H o m}\left(\mathcal{V}, \mathcal{Z}_{D, n}\right)\right)=0$

(2) $\operatorname{Ext}^{1}\left(\mathcal{V}, \mathcal{Z}_{D, n} / \mathcal{V}\right)=H^{1}\left(\mathbb{P}^{1}, \mathcal{H o m}\left(\mathcal{V}, \mathcal{Z}_{D, n} / \mathcal{V}\right)\right)=0$.

Proof. Note the general fact that $\operatorname{Ext}^{1}(\mathcal{F}, \mathcal{G})=H^{1}\left(\mathbb{P}^{1}, \mathcal{H o m}(\mathcal{F}, \mathcal{G})\right)$ if $\mathcal{F}$ is a locally free coherent sheaf on $\mathbb{P}^{1}$ and $\mathcal{G}$ is any coherent sheaf on $\mathbb{P}^{1}$ (see e.g. [16], Chapter III, Propositions 6.3 and 6.7).

It is easy to see that $\mathcal{V}$, being torsion free on a smooth curve, is also locally free. Let $\mathcal{H}=\mathcal{Z}_{D, n}$. By tensoring with a suitable $\mathcal{O}_{\mathbb{P}^{1}}(a)$, we can assume $\mathcal{H}=$ $\mathcal{O}_{\mathbb{P}^{1}}^{k} \oplus \mathcal{O}_{\mathbb{P}^{1}}(-1)^{n-k}$ for some $k>0$. Let $\mathcal{V}=\bigoplus_{i=1}^{r} \mathcal{O}_{\mathbb{P}^{1}}\left(a_{i}\right)$. Clearly, $a_{i} \leq 0$ for $i \in[r]$. Hence $\mathcal{H o m}(\mathcal{V}, \mathcal{H})$ has a decomposition $\bigoplus_{u=1}^{n r} \mathcal{O}_{\mathbb{P}^{1}}\left(b_{u}\right)$ with each $b_{u} \geq-1$. But $H^{1}\left(\mathbb{P}^{1}, \mathcal{O}_{\mathbb{P}^{1}}(b)\right)=0$ for $b \geq-1$. This proves $(1)$. Consider the exact sequence of sheaves

$$
0 \rightarrow \mathcal{V} \rightarrow \mathcal{H} \rightarrow \mathcal{H} / \mathcal{V} \rightarrow 0
$$

which gives an exact sequence

$$
0 \rightarrow \mathcal{H o m}(\mathcal{V}, \mathcal{V}) \rightarrow \mathcal{H o m}(\mathcal{V}, \mathcal{H}) \rightarrow \mathcal{H o m}(\mathcal{V}, \mathcal{H} / \mathcal{V}) \rightarrow 0
$$

Therefore $H^{1}\left(\mathbb{P}^{1}, \mathcal{H} \operatorname{om}(\mathcal{V}, \mathcal{H} / \mathcal{V})\right)$ is surjected on by $H^{1}\left(\mathbb{P}^{1}, \mathcal{H o m}(\mathcal{V}, \mathcal{H})\right)$ (in the Zariski topology, $H^{2}$ of a sheaf on a curve is zero). This proves (2).

9.3. Open subsets in products of flag spaces. We now come to the proof of Lemma 6.5. Let $\mathcal{V}, \mathcal{Q}, \mathcal{I}$ and $\mathcal{K}, d^{\prime}, r^{\prime}, \epsilon$ and $J^{p}$ for $p \in \mathrm{S}$ be as in Section 9.1. Let $\mathcal{U}_{\text {gen }}(\mathcal{V}, \mathcal{Q}, \mathcal{I})$ be the scheme consisting of triples $(\mathcal{F}, \mathcal{G}, \phi)$, where $\mathcal{F} \in \operatorname{Fl}_{\mathrm{S}}(\mathcal{V})$, $\mathcal{G} \in \operatorname{Fl}_{\mathrm{S}}(\mathcal{Q})$ and $\phi \in \operatorname{Hom}_{\mathcal{I}}(\mathcal{V}, \mathcal{Q}, \mathcal{F}, \mathcal{G})$, such that

- $\phi \in \operatorname{Hom}\left(\mathcal{V}, \mathcal{Q}, d^{\prime}, r^{\prime}, \epsilon\right)$,

- if $\mathcal{S}=\operatorname{ker}(\phi)$ and for $p \in \mathrm{S}$, if we set $\mathcal{B}(p)$ to be the kernel of $\bar{\phi}_{p}: \mathcal{V}_{p} / \mathcal{S}_{p} \rightarrow$ $\mathcal{Q}_{p}$, then

$-\mathcal{S} \in \Omega^{o}(\mathcal{K}, \mathcal{V}, \mathcal{F})$

- for $p \in \mathrm{S}, \epsilon(p)=\operatorname{dim}(\mathcal{B}(p))$ and $\mathcal{B}(p) \in \Omega_{J^{p}}^{o}\left(F_{\bullet}^{p}(\mathcal{V} / \mathcal{S})\right)$.

Remark 9.8. The fibre of $\mathcal{U}_{\text {gen }}(\mathcal{V}, \mathcal{Q}, \mathcal{I})$ over $(\mathcal{F}, \mathcal{G}) \in \mathrm{Fl}_{\mathrm{S}}(\mathcal{V}) \times \mathrm{Fl}_{\mathrm{S}}(\mathcal{W})$ is the scheme $\bigcap_{p \in \mathrm{S}} \lambda_{p}^{-1}(R(p)) \subseteq \operatorname{Hom}_{\mathcal{I}}(\mathcal{V}, \mathcal{Q}, \mathcal{F}, \mathcal{G})$ from Section 9.1. Therefore for generic $(\mathcal{F}, \mathcal{G})$ $\in \mathrm{Fl}_{\mathrm{S}}(\mathcal{V}) \times \mathrm{Fl}_{\mathrm{S}}(\mathcal{W})$, this fibre is dense in $\operatorname{Hom}_{\mathcal{I}}(\mathcal{V}, \mathcal{Q}, \mathcal{F}, \mathcal{G}$ ) (which explains the subscript "gen").

We will prove the following lemma in Section 9.4.

Lemma 9.9. (i) The natural morphism $\mathcal{U}_{\text {gen }}(\mathcal{V}, \mathcal{Q}, \mathcal{I}) \rightarrow \mathcal{U}(\mathcal{K}, \mathcal{V})$ is smooth and dominant with connected fibres.

(ii) $\mathcal{U}_{\text {gen }}(\mathcal{V}, \mathcal{Q}, \mathcal{I})$ is a smooth and connected scheme.

Remark 9.10. The possible non-surjectivity of $\mathcal{U}_{\text {gen }}(\mathcal{V}, \mathcal{Q}, \mathcal{I}) \rightarrow \mathcal{U}(\mathcal{K}, \mathcal{V})$ is related to the dominant morphism $a$ from the diagram (9.7) being (possibly) non-surjective. 
It follows from our assumptions that $\pi: \mathcal{U}_{\text {gen }}(\mathcal{V}, \mathcal{Q}, \mathcal{I}) \rightarrow \mathrm{Fl}_{\mathrm{S}}(\mathcal{V}) \times \mathrm{Fl}_{\mathrm{S}}(\mathcal{Q})$ is dominant. Let $O(\mathcal{I}) \subseteq \mathrm{Fl}_{\mathrm{S}}(\mathcal{V}) \times \mathrm{Fl}_{\mathrm{S}}(\mathcal{Q})$ be the largest non-empty open subset such that

- $\pi^{-1}(O(\mathcal{I})) \rightarrow O(\mathcal{I})$ is flat (hence open) and surjective,

- for $(\mathcal{F}, \mathcal{G}) \in O(\mathcal{I})$, the intersection $\bigcap_{p \in \mathrm{S}} \lambda_{p}^{-1}(R(p))$ from Section 9.1 has the expected dimension and is dense in $\operatorname{Hom}_{\mathcal{I}}(\mathcal{V}, \mathcal{Q}, \mathcal{F}, \mathcal{G})$.

We will now construct the desired objects (1) and (2) whose existence was claimed in Lemma 6.5. The Schubert state $\mathcal{K}(\mathcal{I})$ required is just $\mathcal{K}$ from above. We will construct $A(\mathcal{V}, \mathcal{Q})$ inductively and so that it is stable under automorphisms of $\mathcal{V}$ and $\mathcal{Q}$.

If $r=1$, define $A(\mathcal{V}, \mathcal{Q})=\mathrm{Fl}_{\mathrm{S}}(\mathcal{V})^{s} \times B(\mathcal{Q})$. Assume that the construction has been achieved if $r<r_{0}$ and we extend it to the case $r=r_{0}$. The conditions (A) and $(\mathrm{B})$ in Lemma 6.5 are over a finite set of choices of $\mathcal{I}$. It is enough to satisfy the conditions for each $\mathcal{I}$, obtain open subsets $A(\mathcal{V}, \mathcal{Q}, \mathcal{I})$ and finally set

$$
A(\mathcal{V}, \mathcal{Q})=\bigcap_{\mathcal{I}} A(\mathcal{V}, \mathcal{Q}, \mathcal{I})
$$

Fix a Schubert state $\mathcal{I}$ and set $\mathcal{K}=\mathcal{K}(\mathcal{I})=\left(d^{\prime}(\mathcal{I}), r^{\prime}(\mathcal{I}), d, r, K\right)$. Consider the diagram

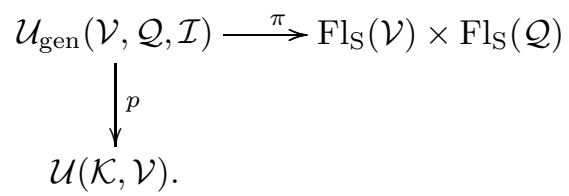

Here $p$ is dominant (Lemma 9.9). Let $U$ be the open subset of $\mathcal{U}(\mathcal{K}, \mathcal{V})$ consisting of points $(\mathcal{S}, \mathcal{F})$ such that

(a) $\mathcal{S}$ and $\mathcal{V} / \mathcal{S}$ are evenly split,

(b) if $0<r^{\prime}(\mathcal{I})<r$, then $(\mathcal{F}(\mathcal{S}), \mathcal{F}(\mathcal{V} / \mathcal{S})) \in A(\mathcal{S}, \mathcal{V} / \mathcal{S})$.

(Use Lemma 4.5 to see that condition (a) is a non-empty open condition. Use Lemma 7.1 in [6] to show the non-emptiness of the open locus of points satisfying (b).)

We finally define

$$
A(\mathcal{V}, \mathcal{Q}, \mathcal{I})=\pi\left(p^{-1}(U)\right) \cap O(\mathcal{I}) \cap(B(\mathcal{V}) \times B(\mathcal{Q}))
$$

which is clearly an open and non-empty subset of $\mathrm{Fl}_{\mathrm{S}}(\mathcal{V}) \times \mathrm{Fl}_{\mathrm{S}}(\mathcal{Q})$ that satisfies the conditions (A) and (B) in Lemma 6.5.

9.4. Proof of Lemma 9.9. Let $\mathcal{I}, \mathcal{K}, \epsilon, d, r$ be as in Section 9.3. Clearly (1) implies (2) in view of Proposition 4.8 (2).

Let

$$
p: \mathcal{U}_{\text {gen }}(\mathcal{V}, \mathcal{Q}, \mathcal{I}) \rightarrow \mathcal{U}(\mathcal{K}, \mathcal{V})
$$

be the natural morphism, let $\mathcal{Y}$ be the universal quotient on $\mathbb{P}^{1} \times \operatorname{Gr}\left(d^{\prime}, r^{\prime}, \mathcal{V}\right)$ and let $\prod_{p \in \mathrm{S}} \operatorname{Gr}\left(\epsilon(p), \mathcal{Y}_{p}\right)$ be the fibre product over $\operatorname{Gr}\left(d^{\prime}, r^{\prime}, \mathcal{V}\right)$, of the Grassmann bundles $\operatorname{Gr}\left(\epsilon(p), \mathcal{Y}_{p}\right)$ (as in Lemma 9.5). Note that $\prod_{p \in \mathrm{S}} \operatorname{Gr}\left(\epsilon(p), \mathcal{Y}_{p}\right)$ consists of pairs $(\mathcal{S}, \mathcal{B})$ where $\mathcal{S} \in \operatorname{Gr}\left(d^{\prime}, r^{\prime}, \mathcal{V}\right)$ and $\mathcal{B}=(\mathcal{B}(p) \mid p \in \mathrm{S}) \in \prod_{p \in \mathrm{S}} \operatorname{Gr}\left(\epsilon(p), \mathcal{V}_{p} / \mathcal{S}_{p}\right)$.

Let $\widetilde{\mathcal{U}}(\mathcal{K}, \mathcal{V})$ be the scheme parameterizing triples $(\mathcal{S}, \mathcal{F}, \mathcal{B})$ where $(\mathcal{S}, \mathcal{F}) \in$ $\mathcal{U}(\mathcal{K}, \mathcal{V})$ and $\mathcal{B}=(\mathcal{B}(p) \mid p \in \mathrm{S}) \in \prod_{p \in \mathrm{S}} \operatorname{Gr}\left(\epsilon(p), \mathcal{V}_{p} / \mathcal{S}_{p}\right)$ such that for every $p \in \mathrm{S}$, $\mathcal{B}(p) \in \Omega_{J^{p}}^{o}\left(F_{\bullet}^{p}(\mathcal{V} / \mathcal{S})\right)$. Clearly $\widetilde{\mathcal{U}}(\mathcal{K}, \mathcal{V}) \rightarrow \mathcal{U}(\mathcal{K}, \mathcal{V})$ is smooth with connected fibres. 
Define $\mathcal{C}$ by the Cartesian square

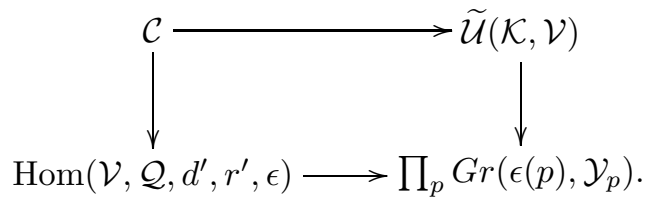

It is easy to see that $\mathcal{C}$ parameterizes triples $(\mathcal{S}, \phi, \mathcal{F})$ such that

- $(\mathcal{S}, \mathcal{F}) \in \mathcal{U}(\mathcal{K}, \mathcal{V})$

- $\operatorname{ker}(\phi)=\mathcal{S}, \phi \in \operatorname{Hom}\left(\mathcal{V}, \mathcal{Q}, d^{\prime}, r^{\prime}, \epsilon\right)$,

- for each $p \in \mathrm{S}$, the kernel of the morphism $\bar{\phi}_{p}: \mathcal{V}_{p} / \mathcal{S}_{p} \rightarrow \mathcal{Q}_{p}$ is in the Schubert cell $\Omega_{J^{p}}^{o}\left(F^{p}(\mathcal{V} / \mathcal{S})\right) \subseteq \operatorname{Gr}\left(\epsilon(p), \mathcal{V}_{p} / \mathcal{S}_{p}\right)$.

By Lemma 9.5, the morphism $\operatorname{Hom}\left(\mathcal{V}, \mathcal{Q}, d^{\prime}, r^{\prime}, \epsilon\right) \rightarrow \prod_{p} G r\left(\epsilon(p), \mathcal{Y}_{p}\right)$ is smooth and dominant with connected fibres. Therefore the base change $\mathcal{C} \rightarrow \widetilde{\mathcal{U}}(\mathcal{K}, \mathcal{V})$ has the same properties. We will now prove that $\mathcal{U}_{\text {gen }}(\mathcal{V}, \mathcal{Q}, \mathcal{I}) \rightarrow \mathcal{C}$ is smooth and surjective with connected fibres. This will conclude the proof of Lemma 9.9.

We will argue "at the level of points" (to be rigorous, we should work at the level of fibres of functors as in the previous section, but we leave this to the reader). The fibre of $\mathcal{U}_{\text {gen }}(\mathcal{V}, \mathcal{Q}, \mathcal{I}) \rightarrow \mathcal{C}$ over $(\mathcal{S}, \phi, \mathcal{F}) \in \mathcal{C}$ is just the set of $\mathcal{G}=\left(G_{\bullet}^{p} \mid p \in\right.$ $\mathrm{S}) \in \mathrm{Fl}_{\mathrm{S}}(\mathcal{Q})$ such that for $p \in \mathrm{S}$ and $a \in[r], \phi_{p}\left(F_{a}^{p}\right) \subseteq G_{i_{a}^{p}-a}^{p}$. The dimension of $\phi_{p}\left(F_{a}^{p}\right)$ can be expressed by a formula in terms of the given data (independent of $\phi)$. Therefore using Lemma A.3 in [6], the set of $\mathcal{G}$ is parameterized by a smooth and connected scheme whose dimension does not jump as we vary $(\mathcal{S}, \phi, \mathcal{F})$. This concludes the proof of Lemma 9.9.

\section{Transversality}

Proposition 10.1. Let $\mathcal{W}$ be an evenly split vector bundle of degree $-D$ and rank $n$ on $\mathbb{P}^{1}$, and let $\mathcal{I}$ be a Schubert state of the form $\mathcal{I}=(d, r, D, n, I)$. Then for generic $\mathcal{E} \in \mathrm{Fl}_{\mathrm{S}}(\mathcal{W})$,

(1) $\Omega^{\circ}(\mathcal{I}, \mathcal{W}, \mathcal{E})$ is a (possibly empty) proper intersection which is transverse on an open dense subset,

(2) if $\operatorname{dim} \mathcal{I}=0, \Omega^{\circ}(\mathcal{I}, \mathcal{W}, \mathcal{E})$ is a smooth and transverse intersection of dimension 0 .

Remark 10.2. The proper intersection statement in (1) follows from Kleiman's theorem.

Proof. If $\mathcal{I}$ is null, there is nothing to prove, since for generic $\mathcal{E}, \Omega^{\circ}(\mathcal{I}, \mathcal{W}, \mathcal{E})=\emptyset$. Therefore assume that $\mathcal{I}$ is non-null and hence $\operatorname{Gr}(d, r, \mathcal{W}) \neq \emptyset$.

Using Proposition 2.2, pick a point $\mathcal{V} \in \operatorname{Gr}(d, r, \mathcal{W})$ such that $\mathcal{V}$ and $\mathcal{Q}=\mathcal{W} / \mathcal{V}$ are both evenly split. Pick a generic point $(\mathcal{F}, \mathcal{G}) \in \mathrm{Fl}_{\mathrm{S}}(\mathcal{V}) \times \mathrm{Fl}_{\mathrm{S}}(\mathcal{Q})$. Now find $\mathcal{E} \in \mathrm{Fl}_{\mathrm{S}}(\mathcal{W})$ such that $\mathcal{V} \in \Omega^{\circ}(\mathcal{I}, \mathcal{W}, \mathcal{E})$ and the flags induced on $\mathcal{V}$ and $\mathcal{Q}$ at points of $\mathrm{S}$ are $\mathcal{F}$ and $\mathcal{G}$, respectively.

Since $\mathcal{I}$ is non-null, condition (B), and hence (C), of Theorem 2.8 holds for the Schubert state $\mathcal{I}$. By the proof of $(\mathrm{C}) \Rightarrow(\mathrm{A})$ in Section 7.1 , the tangent space $\operatorname{Hom}_{\mathcal{I}}(\mathcal{V}, \mathcal{Q}, \mathcal{F}, \mathcal{G})$ of $\Omega^{o}(\mathcal{I}, \mathcal{W}, \mathcal{E})$ at $\mathcal{V}$ is of dimension $\operatorname{dim} \mathcal{I}$. We have therefore found a point $(\mathcal{V}, \mathcal{E}) \in \mathcal{U}(\mathcal{I}, \mathcal{W})$ such that $\Omega^{o}(\mathcal{I}, \mathcal{W}, \mathcal{E})$ is a transverse intersection at $\mathcal{V}$. Such points clearly form an open subset of $\mathcal{U}(\mathcal{I}, \mathcal{W})$. Now apply Lemma 4.9 to the morphism $\mathcal{U}(\mathcal{I}, \mathcal{W}) \rightarrow \mathrm{Fl}_{\mathrm{S}}(\mathcal{W})$ to conclude the proof of (1). If $\operatorname{dim} \mathcal{I}=0$, 
then for generic $\mathcal{E}, \Omega^{o}(\mathcal{I}, \mathcal{W}, \mathcal{E})$ is a finite set. Any dense subset of it is equal to it. Therefore (2) follows from (1).

Theorem 1.6 is a special case of Proposition 10.1 (2). To see this, note that if $D=0, \mathcal{M}_{r, n}^{d}=\operatorname{Gr}\left(d, r, \mathcal{O}_{\mathbb{P}^{1}}^{\oplus n}\right)$ and for generic $\mathcal{E}($ and $\pi$ as in Theorem 1.6),

$$
\pi^{-1}\left(\prod_{j=1}^{s} \Omega_{I^{j}}\left(E_{\bullet}^{j}\right)\right)=\pi^{-1}\left(\prod_{j=1}^{s} \Omega_{I^{j}}^{o}\left(E_{\bullet}^{j}\right)\right)=\Omega^{o}\left(\mathcal{I}, \mathcal{O}_{\mathbb{P}^{1}}^{\oplus n}, \mathcal{E}\right) .
$$

\section{Proof of the Shift properties from Section 2.5}

11.1. Proof of Lemma 2.6. Claim (1) follows from the equality $\chi(d, r, D-d, n-$ $r)=\chi(d+r, r, D-d+n-r, n-r)$.

Fix $T: \mathcal{Z}_{D, n} \otimes \mathcal{O}_{\mathbb{P}^{1}}(-1) \stackrel{\sim}{\rightarrow} \mathcal{Z}_{D+n, n}$ which induces

$$
T^{\prime}: \operatorname{Gr}\left(d, r, \mathcal{Z}_{D, n}\right) \stackrel{\sim}{\rightarrow} \operatorname{Gr}\left(r, d+r, \mathcal{Z}_{D+n, n}\right)
$$

and

$$
\widetilde{T}: \mathrm{Fl}_{\mathrm{S}}\left(\mathcal{Z}_{D, n}\right) \stackrel{\sim}{\rightarrow} \mathrm{Fl}_{\mathrm{S}}\left(\mathcal{Z}_{D+n, n}\right) .
$$

Clearly, we may assume that both $\mathcal{E}$ and $\widetilde{T}(\mathcal{E})$ are generic. For $\mathcal{E} \in \mathrm{Fl}_{\mathrm{S}}\left(\mathcal{Z}_{D, n}\right)$,

$$
T^{\prime}: \Omega^{o}\left(\mathcal{I}, \mathcal{Z}_{D, n}, \mathcal{E}\right) \stackrel{\sim}{\rightarrow} \Omega^{o}\left(\mathcal{J}, \mathcal{Z}_{D+n, n}, \widetilde{T}(\mathcal{E})\right) .
$$

Parts (2) and (3) follow immediately from the isomorphism (11.1).

11.2. Proof of Proposition 2.7. For (1), we consider two cases.

(a) $i_{1}^{p}=1$ : In this case

$$
\operatorname{codim}\left(\omega_{J^{p}}\right)=\operatorname{codim}\left(\omega_{I^{p}}\right)-(n-r), d^{\prime}=d-1
$$

and hence

$$
\operatorname{dim} \operatorname{Gr}\left(d-1, r, \mathcal{Z}_{D-1, n}\right)=\operatorname{dim} \operatorname{Gr}\left(d, r, \mathcal{Z}_{D, n}\right)-(n-r) .
$$

It is immediate that $\operatorname{dim} \mathcal{I}=\operatorname{dim} \operatorname{Sh}(p) \mathcal{I}$.

(b) $i_{1}^{p} \neq 1$ : In this case

$$
\begin{aligned}
\operatorname{codim}\left(\omega_{J^{p}}\right) & =\operatorname{codim}\left(\omega_{I^{p}}\right)+r, \\
d^{\prime} & =d, \\
\operatorname{dim} \operatorname{Gr}\left(d, r, \mathcal{Z}_{D-1, n}\right) & =\operatorname{dim} \operatorname{Gr}\left(d, r, \mathcal{Z}_{D, n}\right)+r
\end{aligned}
$$

and hence

$$
\operatorname{dim} \mathcal{I}=\operatorname{dim} \operatorname{Sh}(p) \mathcal{I} .
$$

We are going to show that if $\mathcal{I}$ is non-null, then $\operatorname{Sh}(p) \mathcal{I}$ is non-null and that $\langle\mathcal{I}\rangle \leq$ $\langle\operatorname{Sh}(p)(\mathcal{I})\rangle$. Since $\operatorname{Sh}(p)^{n} \mathcal{I}=(d-r, r, D-n, n, I)$ where $\mathcal{I}=(d, r, D, n, I)$, the use of iteration and Lemma 2.6 finishes the proof of (2) and (3).

If $\mathcal{I}$ is null, then there is nothing to show. So assume that $\mathcal{I}$ is non-null. Let $\mathcal{W}=\mathcal{Z}_{D, n}$ and choose a generic point $\mathcal{E} \in \mathrm{Fl}_{\mathrm{S}}(\mathcal{W})$. Then $\Omega^{o}(\mathcal{I}, \mathcal{W}, \mathcal{E})$ is non-empty and of dimension $\operatorname{dim}(\mathcal{I})$.

Choose a uniformising parameter $z$ at $p$ and define $\widetilde{\mathcal{W}}$ as follows: $\widetilde{\mathcal{W}}$ agrees with $\mathcal{W}$ outside of $p$. In a neighborhood of $p$, the sections of $\widetilde{\mathcal{W}}$ are meromorphic sections $s$ of $\mathcal{W}$ such that $z s$ is a holomorphic section of $\mathcal{W}$ whose fibre at $p$ is in $E_{1}^{p}$ (this is a special case of the shift operation from Section A.3). Let $\mathcal{J}=\operatorname{Sh}(p) \mathcal{I}$. We claim that $\widetilde{\mathcal{W}}$ is isomorphic to $\mathcal{Z}_{D-1, n}$. The degree computation is clear. We will 
show that $\widetilde{\mathcal{W}}$ is evenly split. Without loss of generality assume that (tensor $\mathcal{W}$ by an appropriate $\left.\mathcal{O}_{\mathbb{P}^{1}}(k)\right)$

$$
\mathcal{W}=\mathcal{O}_{\mathbb{P}^{1}}^{\oplus u} \oplus \mathcal{O}_{\mathbb{P}^{1}}(-1)^{\oplus(n-u)}, u \neq n .
$$

Let $\mathcal{S}=\mathcal{O}_{\mathbb{P}^{1}}^{\oplus u} \subseteq \mathcal{W}$. Since $\mathcal{E} \in \mathrm{Fl}_{\mathrm{S}}(\mathcal{W})$ is generic, we may assume that $E_{1}^{p} \cap \mathcal{S}_{p}=0$.

Let $\widetilde{\mathcal{W}}=\bigoplus_{i=1}^{n} \mathcal{O}_{\mathbb{P}^{1}}\left(a_{i}\right)$. Since $\mathcal{W} \hookrightarrow \widetilde{\mathcal{W}}$, we should have $a_{i} \geq-1$ for $i=1, \ldots, n$. We claim that $a_{i} \leq 0$ for $i=1, \ldots, n$ and this would prove that $\widetilde{\mathcal{W}}$ is evenly split. Assume the contrary, and let $\mathcal{O}_{\mathbb{P}^{1}}(1) \rightarrow \widetilde{\mathcal{W}}$ be a non-zero morphism. Twisting by $\mathcal{O}(-p)$, we have a non-zero morphism $\mathcal{O}_{\mathbb{P}^{1}} \rightarrow \widetilde{\mathcal{W}}(-p)$, or a section $s$ of $\widetilde{\mathcal{W}}$ that vanishes at $p$. Hence $s$ is a global section of $\mathcal{W}$ whose fibre at $p$ is in $E_{1}^{p}$. But this is in contradiction to the assumption that $\mathcal{S}_{p} \cap E_{1}^{p}=0$ ( $s$ is necessarily a section of $\mathcal{S})$. We conclude that $\widetilde{\mathcal{W}}$ is evenly split.

Induce complete flags on the fibres of $\widetilde{\mathcal{W}}$ at each point $q \in \mathrm{S}$ from $\mathcal{E} \in \mathrm{Fl}_{\mathrm{S}}(\mathcal{W})$ (as in [4], Appendix) to obtain $\mathcal{G} \in \mathrm{Fl}_{\mathrm{S}}(\widetilde{\mathcal{W}})$. There is a set-theoretic bijection between the set of subbundles of $\mathcal{W}$ and the set of subbundles of $\widetilde{\mathcal{W}}$. The bijection takes a subbundle $\mathcal{V}$ of $\mathcal{W}$ to the saturation $\widetilde{\mathcal{V}}$ of $\mathcal{V}$ in $\widetilde{\mathcal{W}}$ (see Section A.4). We can keep track of Schubert states under this bijection (for details see [4], Appendix). This tells us that if $\mathcal{V} \in \Omega^{o}(\mathcal{I}, \mathcal{W}, \mathcal{E})$, then $\widetilde{\mathcal{V}} \in \Omega^{o}(\mathcal{J}, \widetilde{\mathcal{W}}, \mathcal{G})$ (and vice versa). From our assumptions $\Omega^{\circ}(\mathcal{I}, \mathcal{W}, \mathcal{E})$ is non-empty of the expected dimension. Since $\operatorname{dim} \mathcal{I}=\operatorname{dim} \mathcal{J}$, we conclude that $\Omega^{o}(\mathcal{J}, \widetilde{\mathcal{W}}, \mathcal{G})$ is also non-empty of the expected dimension. Hence by Lemma 5.6, $\mathcal{J}$ is non-null.

Now assume $\operatorname{dim} \mathcal{I}=0$. The number of points in $\Omega^{o}(\mathcal{I}, \mathcal{W}, \mathcal{E})$ (for $\mathcal{E}$ generic) is exactly $\langle\mathcal{I}\rangle$ and hence we get $\langle\mathcal{I}\rangle$ points of $\Omega^{\circ}(\mathcal{J}, \widetilde{\mathcal{W}}, \mathcal{G})$ where the intersection is proper. This implies that $\langle\mathcal{I}\rangle \leq\langle\mathcal{J}\rangle$ as desired.

\section{Properties of EVEnly Split Vector Bundles}

Lemma 12.1. Let $\mathcal{W}$ be a vector bundle on $\mathbb{P}^{1} \times T$ which is fibrewise, over $T$, of degree $-D$ and rank $n$. Let $t_{0} \in T$ be such that the vector bundle $\mathcal{W}_{t_{0}}$ is evenly split. Then there exists an open subset $U \subseteq T$ containing $t_{0}$ and an isomorphism $\psi: \mathcal{W} \rightarrow p_{\mathbb{P}^{1}}^{*} \mathcal{Z}_{D, n}$ over $U$.

Proof. Choose an isomorphism $s_{0}: \mathcal{W}_{t_{0}} \stackrel{\sim}{\rightarrow} \mathcal{Z}_{D, n}$. Now consider the vector bundle $\mathcal{A}=\mathcal{H} \operatorname{om}\left(\mathcal{W}, p_{\mathbb{P}^{1}}^{*}\left(\mathcal{Z}_{D, n}\right)\right)$ on $\mathbb{P}^{1} \times T$. By Lemma 9.7, $H^{1}\left(\mathbb{P}^{1}, \mathcal{A}_{t_{0}}\right)=0$ and therefore using standard facts on extension of sections (see e.g. [16], Chapter III, Theorem 12.11), $s_{0} \in H^{0}\left(\mathbb{P}^{1}, \mathcal{A}_{t_{0}}\right)$ extends to a neighborhood $U$ of $t_{0}$. We therefore obtain a morphism $\psi: \mathcal{W} \rightarrow p_{\mathbb{P} 1}^{*} \mathcal{Z}_{D, n}$ over $U$, which restricts to the isomorphism $s_{0}$ on the fibre over $t_{0}$. We just need to shrink $U$ further to make $\psi$ an isomorphism.

Lemma 12.2. For every triple of integers $(\lambda, D, n)$ with $n>0$, there exist a smooth and connected scheme $T$, a vector bundle $\mathcal{T}$ on $\mathbb{P}^{1} \times T$ and a non-empty open subset $U_{E S} \subseteq T$ such that

(1) for every vector bundle $\mathcal{W}$ of degree $-D$ and rank $n$ on $\mathbb{P}^{1}$, such that $\mathcal{W}=$ $\sum_{i=1}^{n} \mathcal{O}_{\mathbb{P}^{1}}\left(a_{i}\right)$ with $a_{i} \geq \lambda$ for $i=1, \ldots, n$, there exists $t \in T$ such that $\mathcal{T}_{t}$ is isomorphic to $\mathcal{W}$,

(2) for $t \in T, \mathcal{T}_{t} \stackrel{\sim}{\rightarrow} \mathcal{Z}_{D, n}$ if and only if $t \in U_{E S \text {. }}$ 
Proof. For (1), let us first consider the case $\lambda=0$. Let

$$
T=\operatorname{Ext}^{1}\left(\mathcal{O}_{\mathbb{P}^{1}}(-D), \bigoplus_{i=1}^{n-1} \mathcal{O}_{\mathbb{P}^{1}}\right)
$$

and let $\mathcal{T}$ be the universal extension. Property (1) holds because any $\mathcal{W}$ as in (1) is globally generated and is hence an extension of $\operatorname{det}(\mathcal{W})$ by $\bigoplus_{i=1}^{n-1} \mathcal{O}_{\mathbb{P}^{1}}$ (see e.g. [23], Lemma 8.5.3). We may tensor $\mathcal{T}$ with an appropriate $\mathcal{O}(a)$ to deal with the case $\lambda \neq 0$.

Let $U_{E S}$ be the set of points $t \in T$ such that $\mathcal{T}_{t}$ is evenly split. Clearly by (1), $U_{E S}$ is non-empty. The openness of $U_{E S}$ follows from Lemma 12.1 and we obtain the non-empty open subset $U_{E S}$ with the desired property (2).

12.1. Proof of Proposition 2.2. Consider a point $\mathcal{V} \in \operatorname{Gr}\left(d, r, \mathcal{Z}_{D, n}\right)$. From Lemma 9.7, $\operatorname{Ext}^{1}(\mathcal{V}, \mathcal{H} / \mathcal{V})=0$. Thus (see e.g. [23], Theorem 8.2.1), $\operatorname{Gr}\left(d, r, \mathcal{Z}_{D, n}\right)$ is smooth at $\mathcal{V}$ and the irreducible component of $\operatorname{Gr}\left(d, r, \mathcal{Z}_{D, n}\right)$ passing through $\mathcal{V}$ is of dimension $\chi\left(\mathcal{H o m}\left(\mathcal{V}, \mathcal{Z}_{D, n} / \mathcal{V}\right)\right)=\chi(d, r, D-d, n-r)$.

Consider the subset $U_{G}$ of $\operatorname{Gr}\left(d, r, \mathcal{Z}_{D, n}\right)$ consisting of evenly split subbundles of $\mathcal{Z}_{D, n}$. We are going to show that $U_{G}$ is open, irreducible and dense in $\operatorname{Gr}\left(d, r, \mathcal{Z}_{D, n}\right)$. This will prove the irreducibility of $\operatorname{Gr}\left(d, r, \mathcal{Z}_{D, n}\right)$.

The openness of $U_{G}$ follows from Lemma 12.1. For the irreducibility of $U_{G}$ we argue as follows: Let $Z=\operatorname{Hom}\left(\mathcal{Z}_{d, r}, \mathcal{Z}_{D, n}\right)$. On $\mathbb{P}^{1} \times Z$, we have a universal morphism

$$
\psi: p_{\mathbb{P}^{1}}^{*} \mathcal{Z}_{d, r} \rightarrow p_{\mathbb{P}^{1}}^{*} \mathcal{Z}_{D, n} .
$$

Let $U$ be the largest open subset of $\mathbb{P}^{1} \times Z$ such that the cokernel of $\psi$ is locally free of rank $n-r$ over $U$. Let $U_{Z}=Z-p_{Z}\left(\left(\mathbb{P}^{1} \times Z\right)-U\right)$ which is an open set. If a point $z \in Z$ is in $U_{Z}$, then $\psi_{z}$ is an injective morphism of sheaves on $\mathbb{P}^{1}$ with a locally free cokernel of rank $n-r$. It is easy to see that there is a natural morphism $U_{Z} \rightarrow \operatorname{Gr}\left(d, r, \mathcal{Z}_{D, n}\right)$ and that $U_{G}$ is the image of this morphism. Therefore $U_{G}$ is irreducible.

We will now show that $U_{G}$ is a dense subset of $\operatorname{Gr}\left(d, r, \mathcal{Z}_{D, n}\right)$. Let the point $\mathcal{V} \in$ $\operatorname{Gr}\left(d, r, \mathcal{Z}_{D, n}\right)$ with $\mathcal{V}=\bigoplus_{l=1}^{r} \mathcal{O}_{\mathbb{P}^{1}}\left(a_{l}\right)$ and let $\lambda=\inf _{l} a_{l}-1$. Apply Lemma 12.2 to the triple $(\lambda, d, r)$ and obtain a smooth and connected scheme $T$, a vector bundle $\mathcal{T}$ on $\mathbb{P}^{1} \times T$, and a non-empty open subset $U_{E S} \subseteq T$.

Consider the vector bundle $\mathcal{A}=\mathcal{H o m}\left(\mathcal{T}, p_{\mathbb{P} 1}^{*} \mathcal{Z}_{D, n}\right)$ on $\mathbb{P}^{1} \times T$. Let $t_{0} \in T$ be such that $\mathcal{T}_{t_{0}}$ is isomorphic to $\mathcal{V}$. Now find a section $s_{0} \in H^{0}\left(\mathbb{P}^{1}, \mathcal{A}_{t_{0}}\right)$ that corresponds to the inclusion $\mathcal{V} \subseteq \mathcal{Z}_{D, n}$. According to Lemma 9.7, $H^{1}\left(\mathbb{P}^{1}, \mathcal{A}_{t_{0}}\right)=0$. By Grauert's theorem (see e.g. [16], Chapter III, Corollary 12.9) there exists an open neighborhood $U$ of $t_{0}$ and a section $s$ of $\mathcal{A}$ on $p_{T}^{-1}(U)$ which restricts to $s_{0}$ at $t_{0}$. Said in a different way, there is a morphism $f: \mathcal{T} \rightarrow p_{\mathbb{P}^{1}}^{*} \mathcal{Z}_{D, n}$ on $\mathbb{P}^{1} \times U$ which restricts to the given embedding $\mathcal{V} \subseteq \mathcal{Z}_{D, n}$ on $\mathbb{P}^{1} \times\left\{t_{0}\right\}$. It follows that we can shrink $U$ and assume that $f$ is injective with a locally free cokernel. By the universal property of quot schemes, $f$ corresponds to a morphism $\Theta: U \rightarrow \operatorname{Gr}\left(d, r, \mathcal{Z}_{D, n}\right)$. Now pick a $t^{\prime} \in U \cap U_{E S}$ and restrict $f$ to $\mathbb{P}^{1} \times\left\{t^{\prime}\right\}$. This gives us an embedding $\mathcal{Z}_{d, r} \subseteq \mathcal{Z}_{D, n}$. Therefore $\Theta(U)$ meets $U_{G}$ and contains the point $\mathcal{V} \in \operatorname{Gr}\left(d, r, \mathcal{Z}_{D, n}\right)$. Since $U$ is irreducible, we have thus shown the density of $U_{G}$ in $\operatorname{Gr}\left(d, r, \mathcal{Z}_{D, n}\right)$.

By Grassmann duality $\operatorname{Gr}\left(d, r, \mathcal{Z}_{D, n}\right) \stackrel{\sim}{\rightarrow} \operatorname{Gr}\left(D-d, n-r, \mathcal{Z}_{D, n}^{\vee}\right)$ (which takes $\mathcal{V} \subseteq \mathcal{Z}_{D, n}$ to $\left.(\mathcal{W} / \mathcal{V})^{\vee} \subseteq \mathcal{Z}_{D, n}^{\vee}=\mathcal{Z}_{-D, n}\right)$ and the irreducibility of $\operatorname{Gr}\left(d, r, \mathcal{Z}_{D, n}\right)$, 
the subset of $\operatorname{Gr}\left(d, r, \mathcal{Z}_{D, n}\right)$ consisting of points $\mathcal{V}$ such that both $\mathcal{V}$ and $\mathcal{Z}_{D, n} / \mathcal{V}$ are evenly split, is open and dense. The proof is therefore complete.

Corollary 12.3. The following are equivalent:

(1) $\operatorname{Gr}\left(d, r, \mathcal{Z}_{D, n}\right)$ is non-empty.

(2) $H^{1}\left(\mathbb{P}^{1}, \mathcal{H o m}\left(\mathcal{Z}_{d, r}, \mathcal{Z}_{D-d, n-r}\right)\right)=0$.

Proof. If $\operatorname{Gr}\left(d, r, \mathcal{Z}_{D, n}\right)$ is non-empty, pick $\mathcal{V} \in \operatorname{Gr}\left(d, r, \mathcal{Z}_{D, n}\right)$ such that both $\mathcal{V}$ and $\mathcal{Z}_{D, n} / \mathcal{V}$ are evenly split. We can now apply Lemma 9.7 and deduce $(1) \Rightarrow(2)$.

For $(2) \Rightarrow(1)$, let $\mathcal{V}=\mathcal{Z}_{d, r}$ and $\mathcal{Q}=\mathcal{Z}_{D-d, n-r}$. Let $\lambda$ be such that $(\mathcal{V} \oplus$ $\mathcal{Q}) \otimes \mathcal{O}_{\mathbb{P}^{1}}(-\lambda)$ is globally generated. Apply Lemma 12.2 to the triple $(\lambda, D, n)$ and obtain a smooth and connected scheme $T$, a vector bundle $\mathcal{T}$ on $\mathbb{P}^{1} \times T$ and a nonempty open subset $U_{E S} \subseteq T$. By construction, there is a point $t_{0} \in T$ such that $\mathcal{V} \oplus \mathcal{Q} \stackrel{\sim}{\rightarrow} \mathcal{T}_{t_{0}}$.

Form the relative quot scheme $p: \operatorname{Quot}(\mathcal{T} / T) \rightarrow T$. The fibre of $\operatorname{Quot}(\mathcal{T} / T)$ over a point $t \in T$ is the ordinary quot scheme of quotients of $\mathcal{T}_{t}$ of degree $-(D-d)$ and rank $n-r$. The projection $\pi: \mathcal{V} \oplus \mathcal{Q} \rightarrow \mathcal{Q}$ defines a point of $\operatorname{Quot}(\mathcal{V} \oplus \mathcal{Q})=$ $\operatorname{Quot}(\mathcal{T} / T)_{t_{0}}$. By assumption, $H^{1}\left(\mathbb{P}^{1}, \mathcal{H o m}(\mathcal{V}, \mathcal{Q})\right)=0$; hence the quot scheme $\operatorname{Quot}(\mathcal{V} \oplus \mathcal{Q})$ is smooth at $\pi$ of dimension $\chi\left(\mathbb{P}^{1}, \mathcal{H}\right.$ om $\left.\left(\mathcal{Z}_{d, r}, \mathcal{Z}_{D-d, n-r}\right)\right)$. We now apply standard deformation theory (see e.g. [21] Theorem 5.17) to deduce that $p$ is flat at $\pi$ and hence dominant. We can now conclude the proof because $T$ has a non-empty open subset of points $t$ such that the vector bundle $\mathcal{T}_{t}$ on $\mathbb{P}^{1}$ is evenly split.

\section{Appendix A. Representability CRITERIA AND VECTOR BUNDLES}

A.1. Proof of Proposition 4.8. Let $\mathcal{I}=(d, r, D, n, I)$ be a Schubert state and $\mathcal{W}$ a vector bundle of degree $-D$ and rank $n$ on $\mathbb{P}^{1}$. Let $F(\mathcal{I}, \mathcal{W})$ be the contravariant functor $($ Schemes $/ \kappa) \rightarrow$ (Sets) which assigns to a scheme $T$ over $\kappa$ the set of pairs $(\mathcal{V}, \mathcal{E})$ where

(i) $\mathcal{V}$ is a subbundle of $\mathcal{W}_{T}$ on $\mathbb{P}^{1} \times T$ which is fibrewise, over $\mathrm{T}$, of degree $-d$ and rank $r$ (here $\mathcal{W}_{T}$ is the pull back of $\mathcal{W}$ under the morphism $\mathbb{P}^{1} \times T \rightarrow$ $\left.\mathbb{P}^{1}\right)$,

(ii) $\mathcal{E}=\left(E_{\bullet}^{p} \mid p \in \mathrm{S}\right)$ where $E_{\bullet}^{p}$ is a complete filtration of $\left(\mathcal{W}_{T}\right)_{p}=\left.\left(\mathcal{W}_{T}\right)\right|_{\{p\} \times T}$ by subbundles.

The above data is required to satisfy: For each $p \in \mathrm{S}$, the rank of $\mathcal{V}_{p} \rightarrow\left(\mathcal{W}_{T}\right)_{p} / E_{\ell}^{p}$ (see Section 1.7) is $r-a$ for $i_{a}^{p} \leq \ell<i_{a+1}^{p}, a=0, \ldots, r\left(i_{0}^{p}=0\right.$ and $\left.i_{r+1}^{p}=n\right)$.

Let $T, \mathcal{V}$ and $\mathcal{E}$ be as in the definition above. For $p \in \mathrm{S}$, the vector bundle $\mathcal{V}_{p}$ on $T$ gets a complete induced filtration (by subbundles). The idea is to rewrite the definition of $F(\mathcal{I}, \mathcal{W})$ by including this data. The functor $F(\mathcal{I}, \mathcal{W})$ is naturally equivalent to the functor which assigns to a scheme $T$ over $\kappa$ the set of $\operatorname{data}(\mathcal{V}, \mathcal{E}, \mathcal{F})$ where

(1) $\mathcal{V}$ and $\mathcal{E}$ are as in (i) and (ii) in the definition of the functor $F(\mathcal{I}, \mathcal{W})$,

(2) $\mathcal{F}=\left(F_{\bullet}^{p} \mid p \in \mathrm{S}\right)$ where $F_{\bullet}^{p}$ is a complete filtration of $\mathcal{V}_{p}=\left.\mathcal{V}\right|_{\{p\} \times T}$ by subbundles.

The above data is required to satisfy

(a) for $p \in \mathrm{S}, F_{a}^{p} \subseteq E_{i_{a}^{p}}^{p}$ for $a \in[r]$,

(b) $\mathcal{V}_{p} / F_{a}^{p} \rightarrow\left(\mathcal{W}_{T}\right)_{p} / E_{\ell}^{p}$ is injective with locally free cokernel for $i_{a}^{p} \leq \ell<i_{a+1}^{p}$ and $a \in[r]$. 
If we ignore condition (b), the desired representing scheme is the topmost element $\mathcal{A}$ in a tower of Grassmann bundles ([6], Lemma A.3) over $\operatorname{Fl}_{\mathrm{S}}(\widehat{\mathcal{V}})$ where $\widehat{\mathcal{V}}$ is the universal subbundle on $\operatorname{Gr}(d, r, \mathcal{W})$. The idea is to "choose the flags on $\mathcal{W}$ after you have decided what the induced ones on $\mathcal{V}$ should be". The condition (b) makes the representing scheme an open subscheme of $\mathcal{A}$.

It is now easy to see that this representing scheme coincides at the level of points with $\mathcal{U}(\mathcal{I}, \mathcal{W})$. Clearly $\mathcal{U}(\mathcal{I}, \mathcal{W}) \rightarrow \mathrm{Fl}_{\mathrm{S}}(\widehat{\mathcal{V}})$ is surjective. Since $\mathcal{A}$ is smooth over $\mathrm{Fl}_{\mathrm{S}}(\widehat{\mathcal{V}})$ with connected fibres, conclusion (1) of Proposition 4.8 follows. If $\mathcal{W}$ is evenly split, then by Proposition $2.2, \operatorname{Gr}(d, r, \mathcal{W})$ is smooth and connected. Therefore $\mathrm{Fl}_{\mathrm{S}}(\widehat{\mathcal{V}})$ and hence $\mathcal{A}$ are smooth and connected. Any open subset of a smooth and connected scheme is smooth and connected, and hence conclusion (2) of Proposition 4.8 holds.

A.2. More representability. Let $p: X \rightarrow Y$ be a projective morphism of schemes and let $\mathcal{V}$ be a coherent sheaf on $X$ which is flat over $Y$. Let $F$ be the contravariant functor $($ Schemes $/ Y) \rightarrow($ Sets) which assigns to a scheme $T$ over $Y$ the set $F(T)=$ $H^{0}\left(X_{T}, \mathcal{V}_{T}\right)$ (here $\mathcal{V}$ is the pull back of $\mathcal{V}$ to $X_{T}=X \times_{Y} T$ ). The following lemma is immediate:

Lemma A.1. In the above situation assume that

(a) $p_{*} \mathcal{V}$ is a vector bundle on $Y$,

(b) for any morphism $f: T \rightarrow Y$, if

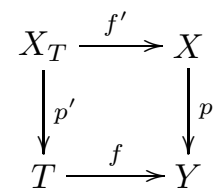

is the base change Cartesian square, the natural morphism $f^{*} p_{*} \mathcal{V} \rightarrow p_{*}^{\prime} f^{\prime *} \mathcal{V}$ is an isomorphism.

Then the functor $F$ is represented by the total space of the vector bundle $p_{*} \mathcal{V}$.

Conditions (a) and (b) in Lemma A.1 hold in each of the following two cases:

(1) $Y=\operatorname{Spec}(\kappa)$. This is because cohomology commutes with flat base change (see e.g. [16], Chapter III, Proposition 9.3).

(2) The higher direct images of $\mathcal{V}$ vanish. In fact it is sufficient to assume that for all points $y \in Y, H^{1}\left(p^{-1}(y), \mathcal{V}_{y}\right)=0$ ([26], Corollary 1 from Lecture 7$)$.

A.3. Shift operations. Fix a point $p \in \mathbb{P}^{1}$. We have a natural inclusion of sheaves $\mathcal{O}_{\mathbb{P}^{1}} \subseteq \mathcal{O}_{\mathbb{P}^{1}}(p)$. Given a uniformising parameter $z$ at $p$, we have an isomorphism $\mathcal{O}_{\mathbb{P}^{1}}(p) \stackrel{z}{\rightarrow} \mathcal{O}_{\mathbb{P}^{1}}$ in a sufficiently small neighborhood of $p$.

Let $\mathcal{V}$ be a vector bundle on $\mathbb{P}^{1} \times T, p \in \mathbb{P}^{1}$ and let $\mathcal{B} \subseteq \mathcal{V}_{p}$ be a subbundle $\left(\mathcal{V}_{p}\right.$ is a vector bundle on $\{p\} \times T)$. There is a canonical inclusion $\mathcal{V} \subseteq \mathcal{V}(p)=\mathcal{V} \otimes p_{\mathbb{P}^{1}}^{*} \mathcal{O}_{\mathbb{P}^{1}}(p)$.

We will define a new "shifted" sheaf $\widetilde{\mathcal{V}}$ such that $\mathcal{V} \subseteq \widetilde{\mathcal{V}} \subseteq \mathcal{V}(p): \widetilde{\mathcal{V}}$ coincides with $\mathcal{V}$ outside of $\left(\mathbb{P}^{1}-\{p\}\right) \times T$ and in a neighborhood of $\{p\} \times T$, it is the kernel of the composite $\mathcal{V}(p) \stackrel{z}{\rightarrow} \mathcal{V} \rightarrow\left(\mathcal{V}_{p} / \mathcal{B}\right)$ where the first morphism is multiplication by $z$. Clearly, $\widetilde{\mathcal{V}}$ is independent of the choice of the uniformising parameter $z$.

It is useful to have a local model of this operation: If $t \in T$, there exists an open subset $U_{t}$ of $T$ containing $t$, a neighborhood $U^{\prime}$ of $\{p\} \times U_{t}$ in $\mathbb{P}^{1} \times T$, and a splitting 
$\mathcal{V}=\mathcal{C} \oplus \mathcal{T}$ on $U^{\prime}$ such that $\mathcal{C}$ restricted to $\{p\} \times U_{t}$ is the same as $\mathcal{B}$. On $U^{\prime}, \widetilde{\mathcal{V}}$ is identified with the subsheaf $\mathcal{C}(p) \oplus \mathcal{T}$ of $\mathcal{V}(p)$.

We can perform the shift operation at various points on $\mathbb{P}^{1}$ simultaneously. Let $\mathcal{V}$ be a vector bundle on $\mathbb{P}^{1} \times T$ and for $p \in \mathrm{S}$, suppose $\mathcal{B}=(\mathcal{B}(p) \mid p \in \mathrm{S})$ where $\mathcal{B}(p) \subseteq \mathcal{V}_{p}$ is a subbundle. Then we form the shift $\Psi(\mathcal{V}, \mathcal{B})$ which satisfies $\mathcal{V} \subset \Psi(\mathcal{V}, \mathcal{B}) \subset \mathcal{V} \otimes p_{\mathbb{P}^{1}}^{*} \mathcal{O}_{\mathbb{P}^{1}}\left(\sum_{p \in S} p\right)$.

Lemma A.2. (1) $\widetilde{\mathcal{V}}$ is locally free, the quotients $\widetilde{\mathcal{V}} / \mathcal{V}$ and $\mathcal{V}(p) / \widetilde{\mathcal{V}}$ are flat over $T$, and the formation of $\widetilde{\mathcal{V}}$ commutes with base change in $T$.

(2) For $p \in \mathrm{S}, \mathcal{V} \rightarrow \widetilde{\mathcal{V}}$ has cokernel $\mathcal{B}(p) \otimes \mathcal{O}_{\mathbb{P}^{1}}(p)$, and $\mathcal{V}_{p} \rightarrow \widetilde{\mathcal{V}}_{p}$ has kernel $\mathcal{B}(p)$.

(3) For any vector bundle $\mathcal{Q}$ on $\mathbb{P}^{1} \times T, \operatorname{Hom}(\widetilde{\mathcal{V}}, \mathcal{Q}) \hookrightarrow \operatorname{Hom}(\mathcal{V}, \mathcal{Q})$ and there is a one to one correspondence between the following objects:

(a) morphisms $\psi: \mathcal{V} \rightarrow \mathcal{Q}$ such that $\mathcal{B}(p) \subseteq \operatorname{ker}\left(\psi_{p}\right)$ for $p \in \mathrm{S}$,

(b) morphisms $\tilde{\psi}: \widetilde{\mathcal{V}} \rightarrow \mathcal{Q}$.

Moreover, for $\psi$ as in (a), $\psi$ is injective with the cokernel flat over $T$, if and only if the same is true for $\tilde{\psi}$.

Proof. This is immediate from the local model of the shift operation. For the flatness assertion use Lemma A.3.

Lemma A.3. Let $\mathcal{V}$ and $\mathcal{Q}$ be vector bundles on $\mathbb{P}^{1} \times T$, and let $\psi: \mathcal{V} \rightarrow \mathcal{Q}$ be a morphism. The following are equivalent:

(1) $\psi$ is injective and the cokernel of $\psi$ is flat over $T$.

(2) For all $t \in T, \psi_{t}$ is injective as a morphism of sheaves.

Proof. (1) $\Rightarrow(2)$ is obvious from the definition of flatness. For $(2) \Rightarrow(1)$ we reason as follows: Let $\mathcal{S}=\operatorname{ker}(\psi)$ and $\mathcal{T}=\operatorname{im}(\psi)$. There are exact sequences

$$
0 \rightarrow \mathcal{S} \rightarrow \mathcal{V} \rightarrow \mathcal{T} \rightarrow 0,0 \rightarrow \mathcal{T} \rightarrow \mathcal{Q} \rightarrow \mathcal{W} \rightarrow 0
$$

For $t \in T, \mathcal{V}_{t} \rightarrow \mathcal{T}_{t}$. The injectivity of $\psi_{t}$ implies that $\mathcal{T}_{t} \rightarrow \mathcal{Q}_{t}$ is also injective, and therefore by the long exact sequence for the Tor functor, $\operatorname{Tor}_{1}^{T}\left(\mathcal{W}, k_{t}\right)=0$ where $k_{t}$ is the residue field of $t$ in $T$.

Therefore, by the local criterion for flatness (see e.g. [9], Theorem 6.8) $\mathcal{W}$ is flat over $T$. The flatness of $\mathcal{Q}$ and $\mathcal{W}$ over $T$ implies that $\mathcal{T}$ and hence $\mathcal{S}$ are also flat over $T$. The injectivity of $\psi_{t}$ implies that $\mathcal{V}_{t} \rightarrow \mathcal{T}_{t}$ is also an injection. Since $\mathcal{T}$ is flat over $T, \mathcal{S}_{t}=0$, and therefore $\mathcal{S}=0$ as desired.

A.4. Saturation of subbundles. Let $\mathcal{W}$ be a vector bundle on a smooth curve and let $\mathcal{V} \subseteq \mathcal{W}$ be a coherent subsheaf. Let $\widetilde{\mathcal{V}} \subseteq \mathcal{W}$ be the inverse image of the torsion subsheaf of $\mathcal{W} / \mathcal{V}$ under the morphism $\mathcal{W} \rightarrow \mathcal{W} / \mathcal{V}$. The sheaf $\widetilde{\mathcal{V}}$, which is a subbundle of $\mathcal{W}$ containing $\mathcal{V}$, is called the saturation of $\mathcal{V}$ in $\mathcal{W}$.

A.5. Parabolic bundles. A parabolic bundle on $\mathbb{P}^{1}$ with parabolic structure along $\mathrm{S}$ is a triple $\mathcal{W}^{\dagger}=(\mathcal{W}, \mathcal{E}, w)$ consisting of a vector bundle $\mathcal{W}$ on $\mathbb{P}^{1}, \mathcal{E} \in \operatorname{Fl}_{\mathrm{S}}(\mathcal{V})$, and a function $w: \mathrm{S} \times[n] \rightarrow \mathbb{R}$ where $n=\operatorname{rk} \mathcal{W}$, such that, denoting $w(p, a)$ by $w_{a}^{p}$,

$$
w_{1}^{p} \geq w_{2}^{p} \geq \cdots \geq w_{n}^{p} \geq w_{1}^{p}-1 .
$$

We define $w_{0}^{p}=w_{n}^{p}+1$. Informally, we will think of $w_{a}^{p}$ as the weight attached to $E_{a}^{p}$. 
For a parabolic bundle $\mathcal{W}^{\dagger}$ as above and a subbundle $\mathcal{V} \subseteq \mathcal{W}$, let $\mathcal{I}=(d, r, D, n, I)$ be the Schubert state such that $\mathcal{V} \in \Omega^{\circ}(\mathcal{I}, \mathcal{W}, \mathcal{E})$.

(1) The parabolic degree of $\mathcal{V}$ is defined to be

$$
\operatorname{pardeg}\left(\mathcal{V}, \mathcal{W}^{\dagger}\right)=-d+\sum_{p \in \mathrm{S}} \sum_{a \in I^{p}} w_{a}^{p} .
$$

(2) If $r \neq 0$, the parabolic slope of $\mathcal{V}$ is defined to be

$$
\mu\left(\mathcal{V}, \mathcal{W}^{\dagger}\right)=\frac{\operatorname{pardeg}\left(\mathcal{V}, \mathcal{W}^{\dagger}\right)}{r}
$$

A parabolic bundle $\mathcal{W}^{\dagger}$ as above is said to be semistable if for all non-zero subbundles $\mathcal{V} \subseteq \mathcal{W}, \mu\left(\mathcal{V}, \mathcal{W}^{\dagger}\right) \leq \mu\left(\mathcal{W}, \mathcal{W}^{\dagger}\right)$.

Lemma A.4. Let $\mathcal{W}^{\dagger}$ be a parabolic bundle as above and let $\psi: \mathcal{V} \hookrightarrow \mathcal{W}$ be a coherent subsheaf of degree $-d$ and rank $r$. Let $\widetilde{\mathcal{V}}$ be the saturation of $\mathcal{V}$ in $\mathcal{W}$. Suppose that there is an $\mathcal{F}=\left(F_{\bullet}^{p} \mid p \in \mathrm{S}\right) \in \mathrm{Fl}_{\mathrm{S}}(\mathcal{V})$ and a function $\gamma: \mathrm{S} \times[r] \rightarrow$ $\{0\} \cup[n]$ such that $\psi_{p}\left(F_{a}^{p}\right) \subseteq E_{\gamma^{p}(a)}^{p}$ for $p \in \mathrm{S}$ and $a \in[r]$. Then

$$
\operatorname{pardeg}\left(\widetilde{\mathcal{V}}, \mathcal{W}^{\dagger}\right) \geq-d+\sum_{p \in \mathrm{S}} \sum_{a=1}^{r} w_{\gamma_{a}^{p}}^{p} .
$$

Proof. Let $\mathcal{B}(p)=\operatorname{ker}\left(\psi_{p}\right)$ and $\epsilon(p)=\operatorname{dim} \mathcal{B}(p)$. Let $\mathcal{I}=\left(d^{\prime}, r, D, n, I\right)$ be the Schubert state such that $\widetilde{\mathcal{V}} \in \Omega^{o}(\mathcal{I}, \mathcal{W}, \mathcal{E})$. It is easy to see that $\operatorname{deg}(\widetilde{\mathcal{V}}) \geq-d+$ $\sum_{p \in \mathrm{S}} \epsilon(p)$ and hence

$$
\operatorname{pardeg}\left(\widetilde{\mathcal{V}}, \mathcal{W}^{\dagger}\right) \geq-d+\sum_{p \in S} \epsilon(p)+\sum_{p \in \mathrm{S}} \sum_{a \in I^{p}} w_{a}^{p} .
$$

Lemma A.4 now follows from

Claim A.5. For each $p \in \mathrm{S}, \epsilon(p)+\sum_{a \in I^{p}} w_{a}^{p} \geq \sum_{a=1}^{r} w_{\gamma^{p}(a)}^{p}$.

For the claim, fix a $p \in \mathrm{S}$ and let $I^{p}=\left\{i_{1}<\cdots<i_{r}\right\}$. Assume $\mathcal{B}(p) \in \Omega_{H}^{o}\left(F_{\text {e }}^{p}\right)$ where $H=\left\{h_{1}<\cdots<h_{\epsilon(p)}\right\}$ and set $\left\{u_{1}<\cdots<u_{r-\epsilon(p)}\right\}=[r] \backslash H$. For $t \in[r-\epsilon(p)], \operatorname{dim} \psi_{p}\left(F_{u_{t}}^{p}\right) \geq t$. This implies that for such $t, i_{t}^{p} \leq \gamma^{p}\left(u_{t}\right)$ and hence $w_{i_{t}^{p}}^{p} \geq w_{\gamma^{p}\left(u_{t}\right)}^{p}$. Therefore

$$
\begin{aligned}
\sum_{a=1}^{r} w_{\gamma^{p}(a)}^{p}-\sum_{a \in I^{p}} w_{a}^{p} & \leq \sum_{\ell=1}^{\epsilon(p)} w_{\gamma^{p}\left(h_{\ell}\right)}^{p}-\sum_{a=r-\epsilon(p)+1}^{r} w_{i_{a}}^{p} \\
& =\sum_{\ell=1}^{\epsilon(p)}\left(w_{\gamma^{p}\left(h_{\ell}\right)}^{p}-w_{i_{r-\epsilon(p)+\ell}}^{p}\right) \\
& \leq \sum_{\ell=1}^{\epsilon(p)} 1=\epsilon(p) .
\end{aligned}
$$

\section{ACKNOWLEDGEMENTS}

I thank A. Buch, W. Fulton, S. Kumar, F. Sottile, C. Woodward and A. Yong for useful communication. I thank the referee for many suggestions on the notation and the exposition. I thank Xiaowei Wang for giving me a copy of Agnihotri's 1995 Ph.D thesis [1]. 


\section{REFERENCES}

1. S. Agnihotri, Quantum cohomology and the verlinde algebra, Ph.D. thesis, University of Oxford, 1995.

2. S. Agnihotri and C. Woodward, Eigenvalues of products of unitary matrices and quantum Schubert calculus, Math. Res. Lett. 5 (1998), no. 6, 817-836. MR1671192 (2000a:14066)

3. Arnaud Beauville, Conformal blocks, fusion rules and the Verlinde formula, Proceedings of the Hirzebruch 65 Conference on Algebraic Geometry (Ramat Gan, 1993) (Ramat Gan), Israel Math. Conf. Proc., vol. 9, Bar-Ilan Univ., 1996, pp. 75-96. MR1360497 (97f:17025)

4. Prakash Belkale, Local systems on $\mathbb{P}^{1}-S$ for $S$ a finite set, Compositio Math. 129 (2001), no. 1, 67-86. MR1856023 (2002k:14085)

5. Transformation formulas in quantum cohomology, Compos. Math. 140 (2004), no. 3, 778-792. MR2041780 (2004m:14119)

6. __ Geometric proofs of Horn and saturation conjectures, J. Algebraic Geom. 15 (2006), no. 1, 133-173. MR2177198 (2006i:14051)

7. Aaron Bertram, Quantum Schubert calculus, Adv. Math. 128 (1997), no. 2, 289-305. MR1454400 (98j:14067)

8. Indranil Biswas, A criterion for the existence of a parabolic stable bundle of rank two over the projective line, Internat. J. Math. 9 (1998), no. 5, 523-533. MR1644048 (99m:14063)

9. David Eisenbud, Commutative algebra with a view toward algebraic geometry, Graduate Texts in Mathematics, vol. 150, Springer-Verlag, New York, 1995. MR1322960 (97a:13001)

10. W. Fulton and R. Pandharipande, Notes on stable maps and quantum cohomology, Algebraic geometry-Santa Cruz, 1995, Proc. Sympos. Pure Math., vol. 62, Amer. Math. Soc., Providence, RI, 1997, pp. 45-96. MR1492534 (98m:14025)

11. W. Fulton and C. Woodward, On the quantum product of Schubert classes, J. Algebraic Geom. 13 (2004), no. 4, 641-661. MR2072765 (2005d:14078)

12. William Fulton, Eigenvalues, invariant factors, highest weights, and Schubert calculus, Bull. Amer. Math. Soc. (N.S.) 37 (2000), no. 3, 209-249 (electronic). MR1754641 (2001g:15023)

13. Eigenvalues of majorized Hermitian matrices and Littlewood-Richardson coefficients, Linear Algebra Appl. 319 (2000), no. 1-3, 23-36, Special Issue: Workshop on Geometric and Combinatorial Methods in the Hermitian Sum Spectral Problem (Coimbra, 1999). MR1799622 (2002a:15024)

14. Doron Gepner, Fusion rings and geometry, Comm. Math. Phys. 141 (1991), no. 2, 381-411. MR1133272 (92j:81271)

15. Foundation of rational conformal field theory 1, hep-th/9211100, 1992.

16. Robin Hartshorne, Algebraic geometry, Springer-Verlag, New York, 1977, Graduate Texts in Mathematics, No. 52. MR0463157 (57:3116)

17. Alfred Horn, Eigenvalues of sums of Hermitian matrices, Pacific J. Math. 12 (1962), 225-241. MR0140521 (25:3941)

18. Steven L. Kleiman, The transversality of a general translate, Compositio Math. 28 (1974), 287-297. MR0360616 (50:13063)

19. Alexander A. Klyachko, Stable bundles, representation theory and Hermitian operators, Selecta Math. (N.S.) 4 (1998), no. 3, 419-445. MR1654578 (2000b:14054)

20. Allen Knutson and Terence Tao, The honeycomb model of $\mathrm{GL}_{n}(\mathbf{C})$ tensor products. I. Proof of the saturation conjecture, J. Amer. Math. Soc. 12 (1999), no. 4, 1055-1090. MR1671451 (2000c:20066)

21. János Kollár, Rational curves on algebraic varieties, Ergebnisse der Mathematik und ihrer Grenzgebiete. 3. Folge. A Series of Modern Surveys in Mathematics, vol. 32, Springer-Verlag, Berlin, 1996. MR1440180 (98c:14001)

22. M. Kontsevich and Yu. Manin, Gromov-Witten classes, quantum cohomology, and enumerative geometry, Mirror symmetry, II, AMS/IP Stud. Adv. Math., vol. 1, Amer. Math. Soc., Providence, RI, 1997, pp. 607-653. MR1416351

23. J. Le Potier, Lectures on vector bundles, Cambridge Studies in Advanced Mathematics, vol. 54, Cambridge University Press, Cambridge, 1997, translated by A. Maciocia. MR1428426 (98a:14019)

24. Hideyuki Matsumura, Commutative ring theory, second ed., Cambridge Studies in Advanced Mathematics, vol. 8, Cambridge University Press, Cambridge, 1989, translated from the Japanese by M. Reid. MR1011461 (90i:13001) 
25. V. B. Mehta and C. S. Seshadri, Moduli of vector bundles on curves with parabolic structures, Math. Ann. 248 (1980), no. 3, 205-239. MR575939 (81i:14010)

26. David Mumford, Lectures on curves on an algebraic surface, with a section by G. M. Bergman. Annals of Mathematics Studies, No. 59, Princeton University Press, Princeton, N.J., 1966. MR0209285 (35:187)

27. Christian Pauly, Espaces de modules de fibrés paraboliques et blocs conformes, Duke Math. J. 84 (1996), no. 1, 217-235. MR1394754 (97h:14022)

28. Alexander Postnikov, Affine approach to quantum Schubert calculus, Duke Math. J. 128 (2005), no. 3, 473-509. MR2145741 (2006e:05182)

29. Frank Sottile, Pieri's formula via explicit rational equivalence, Canad. J. Math. 49 (1997), no. 6, 1281-1298. MR1611668 (2000b:14070)

30. — Real rational curves in Grassmannians, J. Amer. Math. Soc. 13 (2000), no. 2, 333341. MR1706484 (2000j:14080)

31. Elementary transversality in the Schubert calculus in any characteristic, Michigan Math. J. 51 (2003), no. 3, 651-666. MR2021013 (2004i:14065)

32. Ravi Vakil, Schubert induction, Ann. of Math. (2) 164 (2006), no. 2, 489-512. MR2247966

33. Edward Witten, The Verlinde algebra and the cohomology of the Grassmannian, Geometry, topology, \& physics, Conf. Proc. Lecture Notes Geom. Topology, IV, Int. Press, Cambridge, MA, 1995, pp. 357-422. MR1358625 (98c:58016)

34. Alexander Yong, Degree bounds in quantum Schubert calculus, Proc. Amer. Math. Soc. 131 (2003), no. 9, 2649-2655 (electronic). MR1974319 (2004c:14102)

Department of Mathematics, University of North Carolina-Chapel Hill, CB \#3250, Phillips Hall, Chapel Hill, North Carolina 27599

E-mail address: belkale@email.unc.edu 